\title{
Parameter Estimation for the Heat Equation on Perforated Domains
}

\author{
H.T. Banks ${ }^{1}$, D. Cioranescu ${ }^{2}$, A.K. Criner ${ }^{1}$, and W.P. Winfree ${ }^{3}$ \\ ${ }^{1}$ Center for Research in Scientific Computation \\ Department of Mathematics \\ North Carolina State University \\ Raleigh, NC 27695-8212 \\ ${ }^{2}$ Laboratoire J. L. Lions \\ Université Pierre et Marie Curie \\ 75005 Paris, France \\ and \\ ${ }^{3}$ Nondestructive Evaluation Science Branch \\ NASA Langley Research Center, MS 231 \\ Hampton, VA 23681
}

April 23, 2011

\begin{abstract}
In this effort we investigate the behavior of a model derived from homogenization theory as the model solution in parameter estimation procedures for simulated data for heat flow in a porous medium. We consider data simulated from a model on a perforated domain with isotropic flow and data simulated from a model on a homogeneous domain with anisotropic flow. We consider both ordinary and generalized least squares parameter estimation procedures.
\end{abstract}

Key Words: Inverse problems, parameter estimation, perforated domains, homogenization, thermal diffusion, ordinary least squares, generalized least squares 


\section{Introduction}

Nondestructive evaluation is often used to identify damage in structures, including components of aircraft, spacecraft, automobiles, trains and piping, as they age beyond their design life (though there are many other uses, see [15]). Many nondestructive evaluation techniques (acoustic, eddy-current, etc) detect and characterize damages through differences in observed physical parameters, requiring sophisticated parameter estimation procedures. In thermal nondestructive evaluation, damage is often detected and characterized through differences in the observed thermal diffusivity. Nondestructive evaluation has been developed in most cases for homogeneous materials. However many structures of current interest contain components of composite materials which are manufactured with a nontrivial amount of porosity. In [1], we considered modeling the flow of heat through porous samples by use of solutions of a heat equation on a randomly perforated domain. As noted, this model was too computationally intensive for direct use in parameter estimation or inverse problem techniques. We thus considered an approximation for a heat equation on a perforated domain which was derived through limiting processes in homogenization theories for the forward problem in [2]. Though the results of [2] were encouraging, good behavior of an approximation in the forward problem is not necessarily indicative of the behavior of the approximation in inverse problems. Moreover, in our earlier work we did not consider random error which is often associated with experimental data. In this paper we present initial results suggesting that indeed the homogenization approximate mathematical models developed in [2] and [1] will perform well in an inverse problem setting. We do this with noisy simulated data in the context of mathematical and statistical parameter estimation procedures discussed and developed in [6], [13] and also in [4]. We treat data simulated with absolute error in Section 3 and data simulated with relative error in Section 4.

\section{Mathematical Model}

Before formulating a class of inverse problems, we consider several models for the forward problem. We first summarize a method developed in [1] for modeling the flash heat experiment on a porous domain. We consider a randomly perforated domain $\Omega \subset \hat{\Omega}$, where the homogeneous, non perforated domain $\hat{\Omega}$ is an $L_{1} \times L_{2}$ rectangle $\left(L_{1}\right.$ is the length in the horizontal direction). As convention, we take spatial coordinates $(x, y)$, where $x$ is the horizontal coordinate and $y$ is the vertical coordinate. We assume $n_{r}$ randomly placed pores $\Omega_{i}$ with boundaries $\partial \Omega_{i}$ for $i=1,2 \ldots n_{r}$, which are generated using methods described in [1],[2] and [18]. We assume that these pores do not intersect with each other nor the boundaries of $\hat{\Omega}$. The perforated domain $\Omega$ is given by $\hat{\Omega} \backslash\left(\cup_{i=1}^{n_{r}} \Omega_{i}\right)$. The four boundaries of $\hat{\Omega}$ which are also the four exterior boundaries of $\Omega$ are denoted $\omega_{i}$ for $i=1,2,3,4$ (as depicted in Figure 2.1). We model the flash heat experiment which approximates an experiment where the bottom boundary $\omega_{4}=\left\{(x, y) \mid x \in\left[0, L_{1}\right], y=0\right\}$ is heated by a flash heat source [16]. Throughout

this document, we will refer to $\omega_{4}$ as the source boundary. We model the dynamics of the 
flash heat experiment on $\Omega$ with the partial differential equation

$$
\begin{cases}u_{t}^{\text {rand }}-\alpha \nabla \cdot\left(\nabla u^{\text {rand }}\right)+\gamma u^{\text {rand }}=0 & \text { in } \Omega \times(0, T) \\ \alpha \frac{\partial u^{\text {rand }}}{\partial \eta}=0 & \text { on } \cup_{i=1}^{n_{r}} \partial \Omega_{i} \times(0, T) \\ \alpha \frac{\partial u^{\text {rand }}}{\partial \eta}=-\lambda u^{\text {rand }} & \text { on } \cup_{i=1}^{3} \omega_{i} \times(0, T) \\ \alpha \frac{\partial u^{\text {rand }}}{\partial \eta}=S_{f} \mathcal{I}_{\left[0, t_{s}\right]}(t)-\lambda u^{\text {rand }} & \text { on } \omega_{4} \times(0, T) \\ u^{\text {rand }}(0, \vec{x})=0, & \end{cases}
$$

where $\alpha$ is the thermal diffusivity of the material $\Omega, \gamma$ corresponds to loss in the direction orthogonal to the domain $\Omega$ (the $z$ direction) and $\lambda$ corresponds to loss on the boundary of the the rectangle $\hat{\Omega}$.

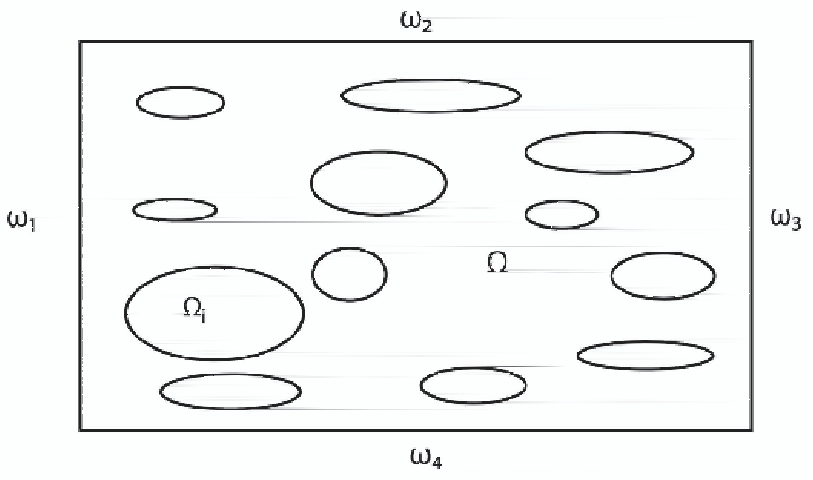

Figure 2.1: An example randomly perforated domain $\hat{\Omega}$ (enlarged view).

The flash heat input is modeled by the term $S_{f} \mathcal{I}_{\left[0, t_{s}\right]}(t)$ where

$$
\mathcal{I}_{\left[0, t_{s}\right]}(t)= \begin{cases}1, & \text { for } t \leq t_{s} \\ 0 & \text { for } t>t_{s}\end{cases}
$$

There are a number of difficulties associated with using (2.1) as a model when carrying out inverse problems. The computational time associated with solving the forward problem (2.1) for $u^{\text {rand }}$ on a time interval of interest in the flash heat experiments is roughly two minutes. This is prohibitively long for use in the inverse problems as well as in some simulation applications of the forward model. Beyond the computational intensity associated with solving (2.1), the random geometry of thin porous samples (which we model as $\Omega$ ) is not precisely known for the nondestructive evaluation (NDE) applications of interest and thus we cannot assume that $\Omega$ is known a priori.

In [2], we discussed the approximation of the heat equation on the random domain $\Omega$ with results derived from homogenization theory (see [2],[8], [11], [9], [12] and [10] and the references therein for details). Using the results of homogenization theory, we obtain the 
limit system

$$
\begin{cases}p_{V} U_{t}-\alpha \nabla \cdot\left(A^{0} \nabla U\right)+\gamma p_{V} U=0 & \text { in } \hat{\Omega} \times(0, T) \\ \alpha \frac{\partial U}{\partial \eta_{A^{0}}}=-\lambda U & \text { on } \cup_{i=1}^{3} \omega_{i} \times(0, T) \\ \alpha \frac{\partial U}{\partial \eta_{A^{0}}}=S_{f} \mathcal{I}_{\left[0, t_{s}\right]}(t)-\lambda U & \text { on } \omega_{4} \times(0, T) \\ U(0, \vec{x})=0, & \end{cases}
$$

where $p_{V}$ is the proportion of $\hat{\Omega}$ occupied by $\Omega\left(p_{V}=\frac{\text { area of } \Omega}{\text { area of } \hat{\Omega}}\right)$ and $A^{0}$ is the $2 \times 2$ homogenized matrix which can be readily calculated using methods described in [2]. On the boundaries we use the notation $\frac{\partial U}{\partial \eta_{A^{0}}}=\vec{n} \cdot A^{0} \nabla U$ where $\vec{n}$ is the exterior unit normal vector. The action of $A^{0}$ is to approximate the isotropic flow through the random domain $\Omega$ around the perforations $\Omega_{i}$ with anisotropic flow through the homogeneous rectangle $\hat{\Omega}$. For more discussion of this aspect of our homogenization approximation, see [2]. Specifically, under reasonable assumptions, one has in particular the following convergences as $n_{r} \rightarrow \infty$ :

$$
\left\{\begin{array}{l}
\tilde{u}^{\text {rand }} \rightarrow p_{V} U \quad \text { weakly in } L^{2}\left(0 ; T ; H^{1}(\hat{\Omega})\right), \\
\nu\left(\tilde{u}^{\text {rand }}\right) \rightarrow \nu(U) \quad \text { weakly in } L^{2}\left(0, T ; L^{2}\left(\omega_{4}\right)\right),
\end{array}\right.
$$

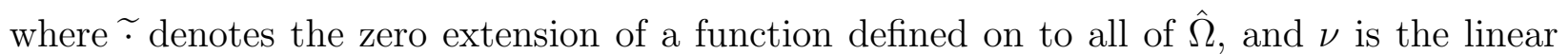
trace operator $\nu: L^{2}\left(0, T ; H^{1}(\hat{\Omega})\right) \mapsto L^{2}\left(0, T ; L^{2}\left(\omega_{4}\right)\right)$. Moreover (for more details see [5]), a corrector result also shows that

$$
\left\{\begin{array}{l}
\lim _{n_{r} \rightarrow \infty}\left\|u^{\text {rand }}-U\right\|_{C\left([0, T] ; L^{2}(\Omega)\right)}=0, \\
\lim _{n_{r} \rightarrow \infty}\left\|\nabla u^{\text {rand }}-C_{\Omega} \nabla U\right\|_{C\left([0, T] ; L^{2}(\Omega)\right)}=0,
\end{array}\right.
$$

where $C_{\Omega}$ is the corrector matrix associated to the elliptic problem corresponding to (2.1). Actually one also has error estimates of the type

$$
\left\|u^{\text {rand }}-U\right\|_{L^{2}(\Omega)} \leq \varepsilon^{1 / 2} C, \quad \text { a. e. for } t \in(0, T) .
$$

Based on this and on the convergences (2.3), (2.4), we propose to use $U$, the solution of (2.2), as a model solution in the ordinary least squares estimation (OLS) procedures with simulated data generated using $U$ with added absolute random error and with simulated data generated using $u^{\text {rand }}$ (the solution of (2.1)) again with added absolute random error in Section 3. In Section 4, we use $U$ as a model solution in the generalized least squares (GLS) estimation procedures with simulated data generated using $U$ with added relative random error and with simulated data generated using $u^{\text {rand }}$ with added relative random error.

\section{Ordinary Least Squares}

We assume a statistical model which describes data with random error that has zero mean, is independent and has constant variance. We discuss the OLS parameter estimation procedure 
in general and then go on to present results from carrying out the OLS parameter estimation procedure on the two different forms of simulated data in Section 3.1.

We consider the full parameter set $\theta=(\gamma, \alpha, \lambda)$, and subsets $\theta^{\lambda}=\left(\gamma_{\lambda}, \alpha_{\lambda}\right)$ (corresponding to the assumption that the boundary loss parameter $\lambda$ is known) and $\theta^{\gamma}=\left(\alpha_{\gamma}, \lambda_{\gamma}\right)$ (corresponding to the assumption that the parameter that models loss in the orthogonal direction $\gamma$ is known). Having introduced the three parameter sets of interest, we will use without loss of generality the notation $\theta^{\#}$ to represent any one of the three parameter sets $\theta, \theta^{\lambda}$ and $\theta^{\gamma}$.

Full state observation is rare, especially when the set of states is continuous (for our problem $\vec{x} \in \hat{\Omega}$ ). Often, when one performs thermal nondestructive evaluation, data is given by the output of an IR camera on the boundary $\omega_{4}$. To model the resulting pixels, we define observation operators

$$
\mathcal{C}_{i}(\phi)=\frac{1}{\ell} \int_{x_{i}}^{x_{i}+\ell} \phi(s, 0) d s .
$$

Thus $\mathcal{C}_{i}$ yields the average value of functions along intervals of length $\ell$ starting at $x=x_{i}$ on $\omega_{4}$ (the source boundary as well as the observation boundary). We suppose the "perfectly resolved" data is given by

$$
U_{i j}\left(\theta_{0}^{\#}\right)=\frac{1}{\ell} \int_{x_{i}}^{x_{i}+\ell} U\left(t_{j}, s, 0 ; \theta_{0}^{\#}\right) d s
$$

or $U_{i j}\left(\theta_{0}^{\#}\right)=\mathcal{C}_{i} U\left(t_{j}, \cdot ; \theta_{0}^{\#}\right)$ where $\theta_{0}^{\#}$ is the "true" parameter value. We will denote $m$ spatial nodes $x_{i}=x_{1}, x_{2}, \ldots, x_{m}$, and $n$ temporal nodes $t_{j}=t_{1}, t_{2}, \ldots, t_{n}$. The statistical assumptions that underlie the OLS parameter estimation procedure corresponding to this observation process is then that data are given by realizations of the random process $\boldsymbol{Y}_{i j}$ which is defined as

$$
\boldsymbol{Y}_{i j}=U_{i j}\left(\theta_{0}^{\#}\right)+\mathcal{E}_{i j}
$$

where $\mathcal{E}_{i j}$ is a random variable that satisfies (3.3) below. The random variable (random error) $\mathcal{E}_{i j}$ is further assumed to have zero mean, be independent and have constant variance. More precisely, we assume

$$
\begin{array}{ll}
E\left(\mathcal{E}_{i j}\right) & =0 \\
\operatorname{Var}\left(\mathcal{E}_{i j}\right) & =\sigma_{0}^{2} \\
\operatorname{Cov}\left(\mathcal{E}_{i j}, \mathcal{E}_{k h}\right) & =0 \text { for }(i, j) \neq(k, h) .
\end{array}
$$

It is important to emphasize that $\boldsymbol{Y}_{i j}$ is a random variable with realizations $y_{i j}$. The realization $y_{i j}$ would correspond to observed data.

In order to obtain the parameter estimate $\hat{\theta}^{\#}$, we must minimize the OLS cost functional

$$
J\left(\theta^{\#}\right)=\sum_{i=1}^{m} \sum_{j=1}^{n}\left(U_{i j}\left(\theta^{\#}\right)-y_{i j}\right)^{2} .
$$

So for each data set $\left\{y_{i j}\right\}$, the parameter estimate is given by

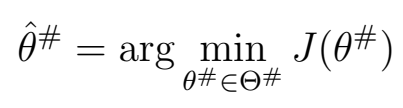


where $\Theta^{\#}$ is an admissible parameter set. The variance estimate is then given by

$$
\hat{\sigma}_{\#}^{2}=\frac{J\left(\hat{\theta}^{\#}\right)}{n m-p}
$$

where $p$ is the number of parameters; thus $p=2$ for $\hat{\theta}^{\#}=\hat{\theta}^{\gamma}$ and $\hat{\theta}^{\#}=\hat{\theta}^{\lambda}$ while $p=3$ for $\hat{\theta}^{\#}=\hat{\theta}$.

Data collection nodes can be selected in many sophisticated ways such as by using SEoptimal design, E-optimal design, D-optimal and c-optimal design methods (see [7] and references therein). However, this is not the focus of our current efforts so we will simply examine the traditional sensitivity functions to select data collections nodes. For each parameter $\theta_{k}^{\#}$ in the parameter set $\theta^{\#}$, the associated traditional sensitivity function (the sensitivity of the model solution with respect to $\left.\theta_{k}^{\#}\right) V_{k}^{\theta_{k}^{\#}}=\frac{\partial U}{\partial \theta_{k}^{\#}}$ corresponds to the sensitivity of the solution with respect to the $k$ th parameter. In places where the sensitivity $V^{\theta_{k}^{\#}}$ is zero, one cannot obtain any information about the $k$ th parameter. However, sensitivity information must be used with care in design of inverse problems [3]. For example, the information is local in nature (i.e., depends on the values $\theta^{\#}$ at which the derivatives are evaluated) and moreover, one should not exclusively choose nodes in the regions of the highest sensitivity.

We examined the output sensitivity functions

$$
\begin{aligned}
V^{\gamma}\left(t, x_{i}\right) & =\frac{\partial}{\partial \gamma}\left(\frac{1}{\ell} \int_{x_{i}}^{x_{i}+\ell} U\left(t, s, 0 ;\left(10^{-3}, 2.9167,0.01\right)\right) d s\right) \\
V^{\alpha}\left(t, x_{i}\right) & =\frac{\partial}{\partial \alpha}\left(\frac{1}{\ell} \int_{x_{i}}^{x_{i}+\ell} U\left(t, s, 0 ;\left(10^{-3}, 2.9167,0.01\right)\right) d s\right) \\
V^{\lambda}\left(t, x_{i}\right) & =\frac{\partial}{\partial \lambda}\left(\frac{1}{\ell} \int_{x_{i}}^{x_{i}+\ell} U\left(t, s, 0 ;\left(10^{-3}, 2.9167,0.01\right)\right) d s\right)
\end{aligned}
$$

for $\ell=0.57$ and $x_{i}=0,0.57,1.14,1.71$ using calculations detailed in Appendix A. We chose the values of $x_{i}$ and $\ell$ based on an example pixel width. For $\theta^{\#}$ we chose $\gamma=10^{-3}$, $\alpha=2.9167$ and $\lambda=0.01$ based on physically reasonable values [2]. These are also the values of $\theta^{\#}$ that we used for the simulated data in Sections 3.1 and 4.1.

After inspecting $V^{\gamma}, V^{\alpha}$ and $V^{\lambda}$ for different values of $x_{i}$, we observed little difference and thus we arbitrarily chose two sets of spatial nodes $x_{i} \in\{0,0.57\}$ and $x_{i} \in\{0,0.57,1.14\}$ to consider the effect of sparsity of spatial nodes on the inverse problem. As depicted in Figures 3.1(a) and (b), the values of $V^{\gamma}\left(t, x_{i}\right), V^{\alpha}\left(t, x_{i}\right)$ and $V^{\lambda}\left(t, x_{i}\right)$ vary over time. The sensitivity with respect to $\alpha, V^{\alpha}\left(t, x_{i}\right)$ goes to zero quickly after the end of flash-heating at $t_{s}=0.6 \mathrm{~s}$ in Figure 3.1(a). There is often more measurement error during flash-heating so we take many times just after the end of flash heating $\left\{0.6+\frac{1}{120}, 0.6+\frac{2}{120}, \ldots 0.6+\frac{7}{120}\right\}$ to gain information about the parameter $\alpha$. The sensitivities to $\gamma$ and $\lambda$ are depicted in Figure 3.1(b) and go to zero much more slowly than $V^{\alpha}$. We take times $\{20,40, \ldots, 140\}$ to gain information about the parameters $\gamma$ and $\lambda$. We note that in Figure 3.1(b) the sensitivity with respect to $\gamma$ is less than the sensitivity with respect to $\lambda$. This suggests 


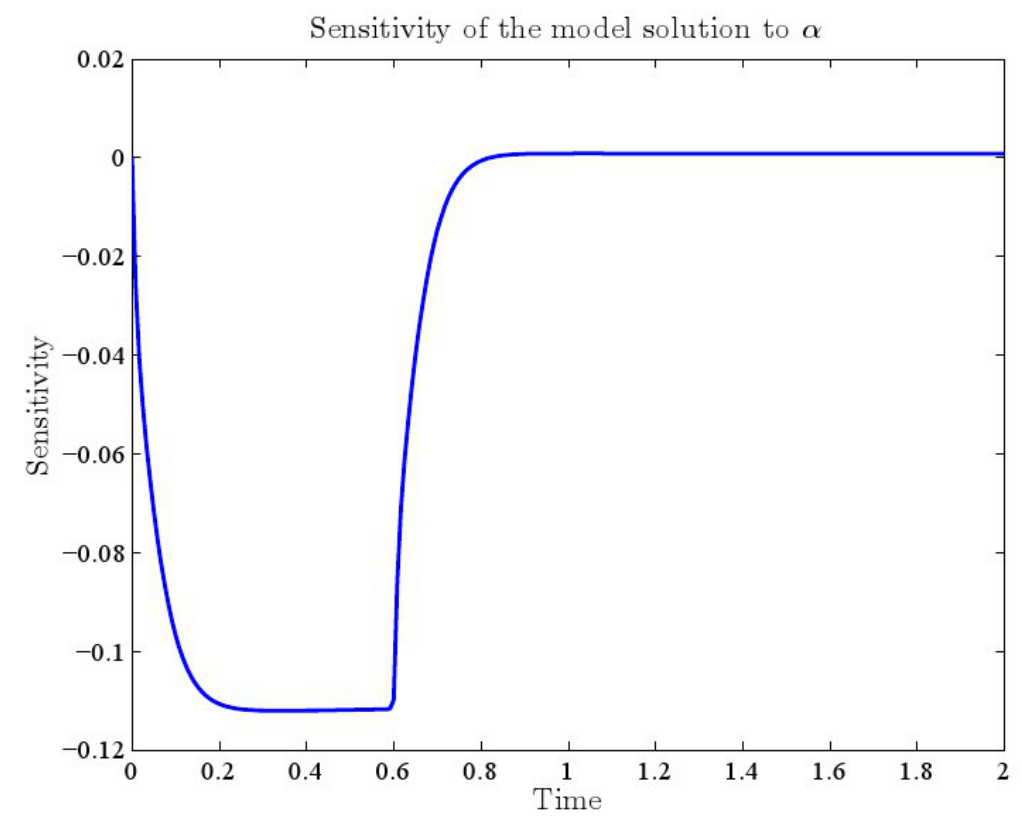

(a)

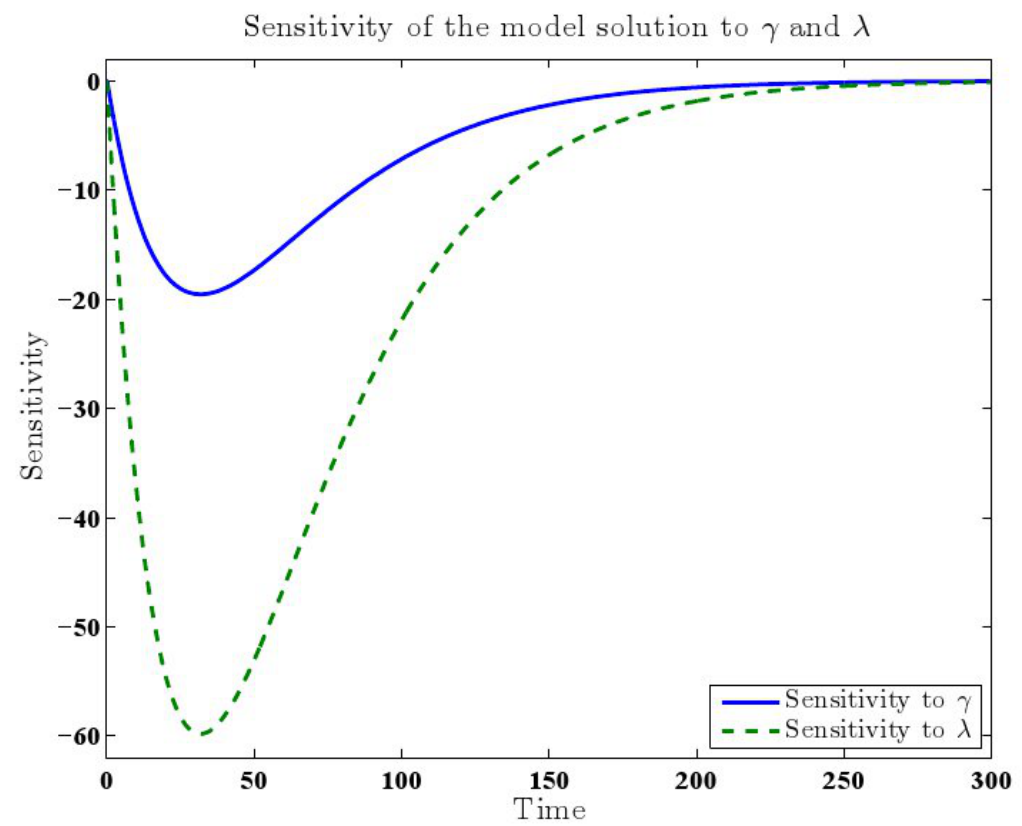

(b)

Figure 3.1: The sensitivity functions of $\frac{1}{0.57} \int_{0}^{0.57} U(t, s, 0) d s$ where $U$ is the solution of $(2.2)$ with $\gamma=10^{-3}, \alpha=2.9167$ and $\lambda=0.01$. (a) The sensitivity with respect to $\alpha$ for time $t \in[0,2]$. (b) The sensitivity with respect to $\lambda$ and $\gamma$ for time $t \in[0,300]$. 
that there could be problems estimating $\gamma$; we will further discuss the well-posedness of estimating $\gamma$ in Section 3.1.

Using the sensitivity matrix with entries

$$
\chi_{i+m(j-1), k}(\theta)=\left.\frac{\partial}{\partial \theta_{k}} U_{i j}(\zeta)\right|_{\zeta=\theta}
$$

we may use asymptotic distribution results (see [6]) to calculate the estimated covariance matrix

$$
\Sigma\left(\hat{\theta}^{\#}\right)=\hat{\sigma}^{2}\left(\chi^{T}\left(\hat{\theta}^{\#}\right) \chi\left(\hat{\theta}^{\#}\right)\right)
$$

and the estimated standard error for the $k$ th parameter

$$
\operatorname{SE}\left(\hat{\theta}_{k}^{\#}\right)=\sqrt{\sum_{k k}\left(\hat{\theta}^{\#}\right)}
$$

\subsection{Simulated Ordinary Least Squares Data}

We would like to consider data motivated by (2.1) because we suspect that this model will generate solutions that resemble experimental data. We cannot, however, use (2.1) as a model solution in the OLS cost functional (3.4) because the random geometry $\Omega$ in (2.1) is not a priori knowledge in most thermal nondestructive evaluation applications. On the other hand we observe that $U_{i j}(\theta)$ of $(2.2)$ depends on $p_{V}$, the porosity ration for which reasonable a priori estimates are available. Thus we are concerned with the behavior of $U_{i j}(\theta)$ as a model solution in the inverse problem with data motivated by $u^{\text {rand }}$. It is not simple to ensure that the OLS assumptions (i.e., model independent constant variance errors that are iid as in (3.3)) are satisfied even in the most straight forward cases because relations between parameters can cause violations in the OLS assumptions. For instance, the estimation of $\gamma$ is ill-posed. In the partial differential equation (2.2), if we make the transformation $U=e^{-\gamma t} Z$ in (2.2) the partial differential equation system becomes

$$
\begin{cases}p_{V} Z_{t}-\alpha \nabla \cdot\left(A^{0} \nabla Z\right)=0 & \text { on } \hat{\Omega} \times(0, T) \\ \alpha \frac{\partial Z}{\partial \eta_{A^{0}}}=-\lambda Z & \text { on } \cup_{i=1}^{3} \omega_{i} \times(0, T) \\ \alpha \frac{\partial Z}{\partial \eta_{A^{0}}}=S_{f} \tilde{\mathcal{I}}_{\left[0, t_{s}\right]}(t)-\lambda Z & \text { on } \omega_{4} \times(0, T),\end{cases}
$$

where $\tilde{\mathcal{I}}_{\left[0, t_{s}\right]}(t)=e^{\gamma t} \mathcal{I}_{\left[0, t_{s}\right]}(t)$. This means that if we rewrite the cost functional in (3.4) as

$$
J(\theta)=\sum_{i=1}^{m} \sum_{j=1}^{n}\left(\mathcal{C}_{i} U\left(t_{j}, \cdot ; \theta\right)-\mathrm{d}_{i j}\right)^{2}
$$

we can see that the cost functional $J(\theta)$ in $(3.12)$ where $U\left(t_{j}, \cdot ; \theta\right)$ is the solution of $(2.2)$ can equivalently be written as the weighted least squares

$$
J(\theta)=\sum_{i=1}^{m} \sum_{j=1}^{n} e^{-2 \gamma t_{j}}\left(\mathcal{C}_{i} Z\left(t_{j}, \cdot ; \theta\right)-e^{\gamma t_{j}} \mathrm{~d}_{i j}\right)^{2},
$$


and an equivalent OLS formulation for $Z$ would drop the weight terms $e^{-2 \gamma t_{j}}$. Here $Z(\theta)$ is the solution of (3.11) and $\mathrm{d}_{i j}$ is the observed data. This implies that by including $\gamma$ in the inverse problem formulation, any noise in the data might be amplified. This will also cause model dependence in the error in $\mathrm{d}_{i j}$ which violates the error assumptions of (3.3) in the corresponding OLS formulation. This is evidence that the parameter $\gamma$ plays a very different role in the estimation problems when compared to that of either $\alpha$ or $\lambda$, and along with the sensitivity results of Figure 3.1, suggests an additional level of ill-posedness in estimating $\gamma$ that is not present in problems where $\gamma$ is held fixed.

Because of the subtlety in verifying the OLS error assumptions (3.3) directly, we will compare data simulated using the solution of (2.2) with data simulated using the solution of (2.1). We simulate data motivated by OLS assumptions and the solution of (2.2) with the random process

$$
\mathbf{D}_{i j}(\sigma)=U_{i j}\left(10^{-3}, 2.9167,0.01\right)+\sigma \boldsymbol{B}_{i j}
$$

where $\boldsymbol{B}_{i j}$ is a random variable which follows a standard normal distribution or $\boldsymbol{B}_{i j} \sim$ $\mathcal{N}\left(0,1^{2}\right)$. We consider two sets of spatial nodes $x_{i}=0,0.57$ and $x_{i}=0,0.57,1.14$.

We simulate data motivated by the OLS error assumptions (3.3) and the solution of (2.1) with the random process

$$
\mathbf{D}_{i j}^{\mathrm{rand}}(\sigma)=u_{i j}^{\mathrm{rand}}+\sigma \boldsymbol{B}_{i j}
$$

where $u_{i j}^{\text {rand }}$ is given by

$$
u_{i j}^{\mathrm{rand}}=\frac{1}{\ell} \int_{x_{i}}^{x_{i}+\ell} u^{\mathrm{rand}}\left(t_{j}, s, 0 ;\left(10^{-3}, 2.9167,0.01\right)\right) d s,
$$

with $u^{\text {rand }}\left(t_{j}, s, 0 ;\left(10^{-3}, 2.9167,0.01\right)\right)$ the solution of $(2.1)$ with $(\gamma, \alpha, \lambda)=\left(10^{-3}, 2.9167,0.01\right)$ on a randomly perforated geometry $\Omega$.

For realizations of $\mathbf{D}_{i j}(\sigma)$ and $\mathbf{D}_{i j}^{\text {rand }}(\sigma)$ each data set is analyzed using the results of OLS asymptotic theory for the parameter sets $\theta^{\gamma}=\left(\alpha_{\gamma}, \lambda_{\gamma}\right), \theta^{\lambda}=\left(\gamma_{\lambda}, \alpha_{\lambda}\right)$ and $\theta=(\gamma, \alpha, \lambda)$. So for each data set we preform three inverse problems calculating three parameter estimates $\hat{\theta}^{\gamma}, \hat{\theta}^{\lambda}$ and $\hat{\theta}$ using (3.5), three variance estimates $\hat{\sigma}_{\gamma}^{2}, \hat{\sigma}_{\lambda}^{2}$ and $\hat{\sigma}^{2}$ using (3.6), three covariance matrix estimates $\Sigma\left(\hat{\theta}^{\gamma}\right), \Sigma\left(\hat{\theta}^{\lambda}\right)$, and $\Sigma(\hat{\theta})$ using (3.9), and the standard error for each parameter in the sets $\hat{\theta}^{\gamma}, \hat{\theta}^{\lambda}$ and $\hat{\theta}$, denoted $\operatorname{SE}\left(\hat{\alpha}_{\gamma}\right), \operatorname{SE}\left(\hat{\lambda}_{\gamma}\right), \operatorname{SE}\left(\hat{\gamma}_{\lambda}\right), \operatorname{SE}\left(\hat{\alpha}_{\lambda}\right)$, and $\operatorname{SE}(\hat{\gamma}), \operatorname{SE}(\hat{\alpha}), \operatorname{SE}(\hat{\lambda})$ using (3.10).

There are many different ways to consider these parameter estimation and uncertainty quantification problems. We will consider the difference between the parameter estimate and the "true" parameter values $\gamma_{0}=10^{-3}, \alpha_{0}=2.9167$, and $\lambda_{0}=0.01$ which will give us insight into the accuracy of the parameter estimate $\hat{\theta}_{k}^{\#}$. Recall the ratio $\operatorname{SE}\left(\hat{\theta}_{k}^{\#}\right) / \hat{\theta}_{k}^{\#}$ is related to the uncertainty associated with the parameter estimate $\hat{\theta}_{k}^{\#}$. Thus we also consider the ratio of the estimated standard error to the parameter estimate. When this ratio is large, there is little confidence in the value of the parameter $\hat{\theta}_{k}^{\#}$. For instance, the $95 \%$ confidence interval [6] for $m=3$ for $\hat{\gamma}$ is given by $(\hat{\gamma}-2.02 \times \mathrm{SE}(\hat{\gamma}), \hat{\gamma}+2.02 \times \mathrm{SE}(\hat{\gamma}))$. In this example when $\mathrm{SE}(\hat{\gamma}) / \hat{\gamma}$ is greater than 0.5 , the confidence interval actually covers possible negative values for $\gamma$. 
We compare the results of ordinary least squares parameter estimation procedure for realizations of $\mathbf{D}_{i j}(\sigma)$ with the results of ordinary least squares parameter estimation procedure for realizations of $\mathbf{D}_{i j}^{\text {rand }}(\sigma)$. In Figures 3.2-3.7, the simulated data is taken at temporal collection nodes $t_{j}=0.6+\frac{1}{120}, 0.6+\frac{2}{120}, \ldots, 0.6+\frac{7}{120}, 20,40, \ldots, 140$ and spatial nodes $x_{i}=0,0.57,1.14$. Figure 3.2(a) depicts the difference $\hat{\alpha}_{\#}-\alpha_{0}$ for each of five realizations at each level of added noise $\sigma=0.015,0.030, \ldots, 0.090$ for $\mathbf{D}_{i j}(\sigma)$. The estimates for the three parameter subsets do not appear to vary much. That is, we see that for each realization the differences $\hat{\alpha}-\alpha_{0}, \hat{\alpha}_{\gamma}-\alpha_{0}$ and $\hat{\alpha}_{\lambda}-\alpha_{0}$ are relatively close. Figure 3.2(b) depicts the difference $\hat{\alpha}_{\#}-\alpha_{0}$ for each of five realizations of $\mathbf{D}_{i j}^{\text {rand }}(\sigma)$ at each level of noise $\sigma=0,0.015, \ldots, 0.090$, we see that the differences $\hat{\alpha}_{\#}-\alpha_{0}$ again do not appear to vary much for each realization. Moreover, the results depicted in Figure 3.2(a) resemble the results depicted in Figure 3.2(b). This suggests that using realizations of $\mathbf{D}_{i j}(\sigma)$ versus $\mathbf{D}_{i j}^{\text {rand }}(\sigma)$ does not affect the accuracy of the estimates $\hat{\alpha}_{\#}$. In Figures 3.3 (a) and (b), we examine the ratios $\operatorname{SE}\left(\hat{\alpha}_{\#}\right) / \hat{\alpha}_{\#}$ for the five realizations of $\mathbf{D}_{i j}(\sigma)$ and $\mathbf{D}_{i j}^{\text {rand }}(\sigma)$, respectively. In Figure 3.3(a), we see that the uncertainty associated with the estimate $\hat{\alpha}$ is larger than the uncertainty associated with the estimates $\hat{\alpha}_{\gamma}$ and $\hat{\alpha}_{\lambda}$. It also appears that the ratio $\operatorname{SE}\left(\hat{\alpha}_{\#}\right) / \hat{\alpha}_{\#}$ varies linearly with $\sigma$. These observations are valid for the ratios depicted in Figure 3.3(b), as well. The similarities in Figure 3.3(a) and Figure 3.3(b) suggest that there is not a significant difference in using realizations $\mathbf{D}_{i j}(\sigma)$ and $\mathbf{D}_{i j}^{\text {rand }}(\sigma)$ regarding the uncertainty associated the OLS parameter estimate $\hat{\alpha}_{\#}$.

We also considered the parameter $\lambda$. Figures 3.4(a) are the differences $\lambda_{\#}-\lambda_{0}\left(\lambda_{0}=0.01\right)$ for the realizations used in Figures 3.2(a) and 3.3(a). Unlike the parameter $\alpha$, there is a large difference in the $\hat{\lambda}_{\#}-\lambda_{0}$. The magnitude of the difference $\hat{\lambda}-\lambda_{0}$ is much larger than the magnitude of the difference $\hat{\lambda}_{\gamma}-\lambda_{0}$ indicating that estimating $\gamma$ adds inaccuracy to the estimate of $\lambda$. In Figure 3.4(b), we have plotted the difference $\hat{\lambda}_{\#}-\lambda_{0}$ for the realizations depicted in Figures 3.2(b) and 3.3(b). In Figure 3.4(b), we see that the differences $\hat{\lambda}_{\gamma}-\lambda_{0}$ and $\hat{\lambda}-\lambda_{0}$ for the realizations of $\mathbf{D}_{i j}^{\text {rand }}(\sigma)$ resemble these differences for the realizations of $\mathbf{D}_{i j}(\sigma)$ in Figure 3.4(a) so we suspect that the error associated with the approximation of $u^{\text {rand }}$ (the solution of (2.1)) with $U$ (the solution of (2.2)) in the model solution does not affect the estimate of $\hat{\lambda}_{\gamma}$ nor that of $\hat{\lambda}$.

In Figure 3.5(a), we see that the ratio $\operatorname{SE}\left(\hat{\lambda}_{\gamma}\right) / \hat{\lambda}_{\gamma}$ appears to be linear in $\sigma$ for the realizations of $\mathbf{D}_{i j}(\sigma)$. This linear pattern is similar to the linearity in Figure 3.5(c) which depicts the ratio $\operatorname{SE}\left(\hat{\lambda}_{\gamma}\right) / \hat{\lambda}_{\gamma}$ for the realizations of $\mathbf{D}_{i j}^{\text {rand }}(\sigma)$. Moreover, the ratio $\operatorname{SE}\left(\hat{\lambda}_{\gamma}\right) / \hat{\lambda}_{\gamma}$ is on about the same scale in Figures 3.5(a) and (c). So for $\hat{\lambda}_{\gamma}$, there does not appear to be a significant difference in using data generated by (3.14) versus data generated by (3.15) in the accuracy of the parameter estimate $\hat{\lambda}_{\gamma}$ nor in the uncertainty associated with the parameter estimate $\hat{\lambda}_{\gamma}$.

In order to consider the ratio $\operatorname{SE}(\hat{\lambda}) / \hat{\lambda}$, we plotted the logarithm of this quantity in Figures 3.5(b) (for realizations of $\mathbf{D}_{i j}(\sigma)$ ) and 3.5(d) (for realizations of $\mathbf{D}_{i j}^{\text {rand }}(\sigma)$ ) because in both examples these quantities vary greatly. The ratio $\operatorname{SE}(\hat{\lambda}) / \hat{\lambda}$ in both Figure $3.5(\mathrm{~b})$ and Figure 3.5(d) appears to grow exponentially with added error $\sigma$ which is not surprising 


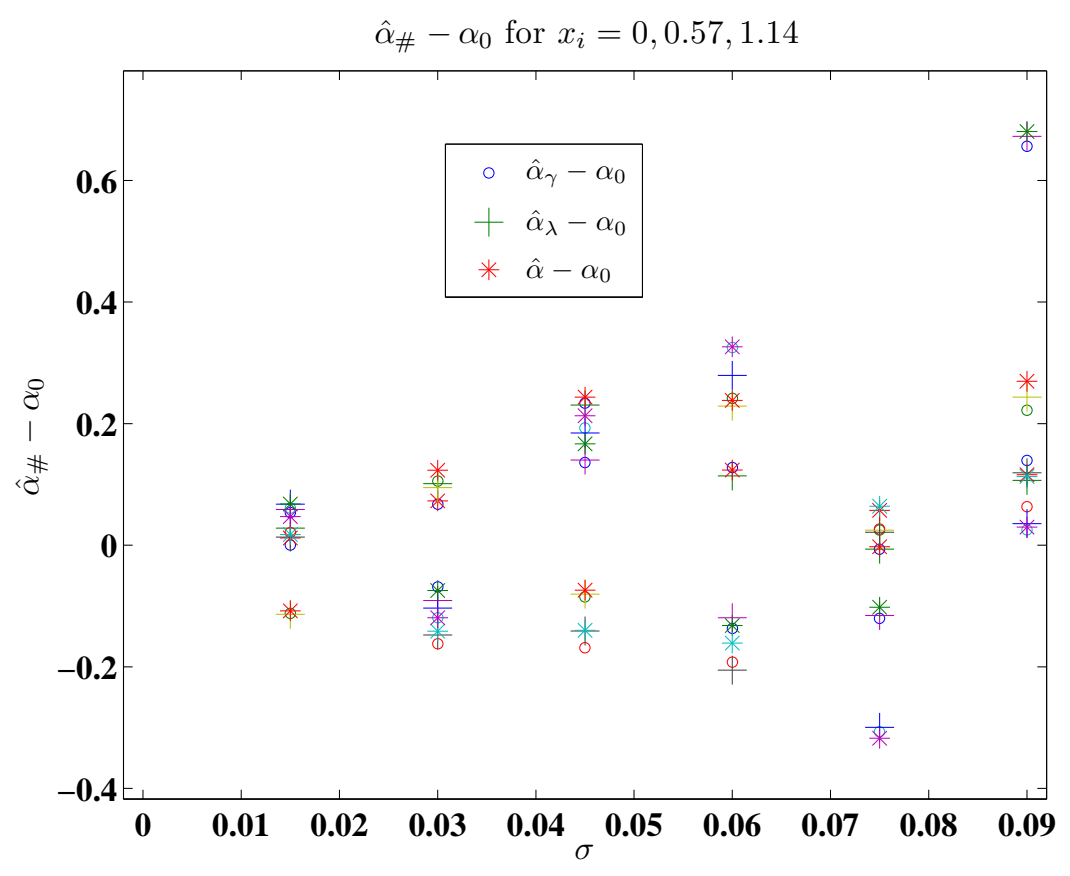

(a)

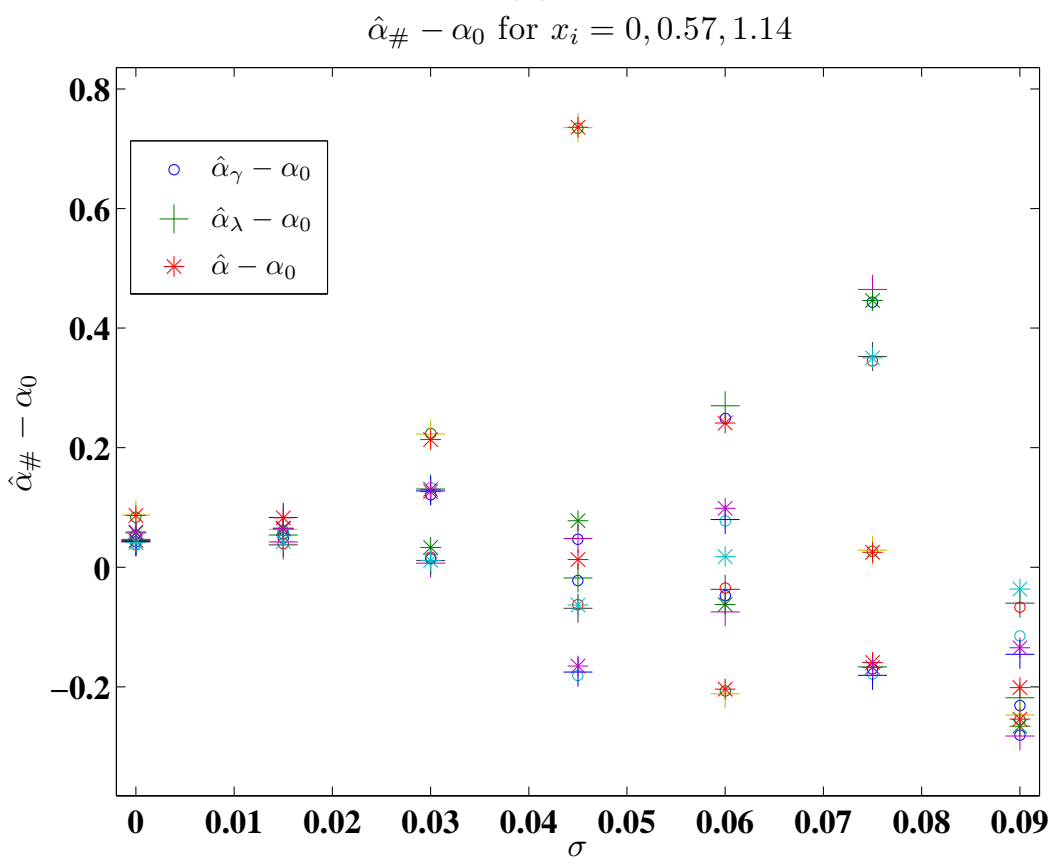

(b)

Figure 3.2: The results of realizations of simulated data with temporal nodes $t_{j}=$ $0.6+\frac{1}{120}, 0.6+\frac{2}{120}, \ldots, \frac{7}{120}, 20,40, \ldots, 140$ and spatial nodes $x_{i}=0,0.57,1.14$. The points denoted with ${ }^{o}$ are the difference $\hat{\alpha}_{\gamma}-\alpha_{0}$, the points denoted are the $\hat{\alpha}_{\lambda}-\alpha_{0}$, the points denoted * are the difference $\hat{\alpha}-\alpha_{0}$ (a) The result of five realizations of $\mathbf{D}_{i j}(\sigma)$ for values of $\sigma=0.015,0.030, \ldots, 0.090$ (b) The result of five realizations of $\mathbf{D}_{i j}^{\text {rand }}(\sigma)$ for values of $\sigma=0,0.015, \ldots, 0.090$. 


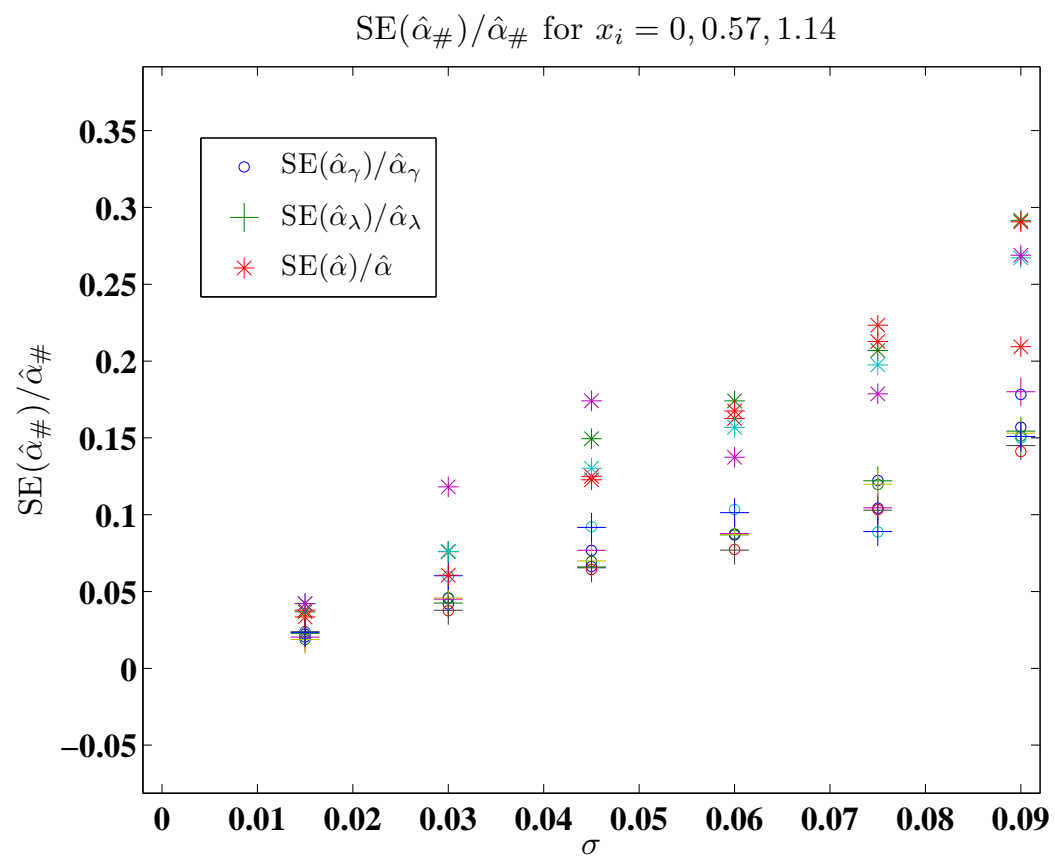

(a)

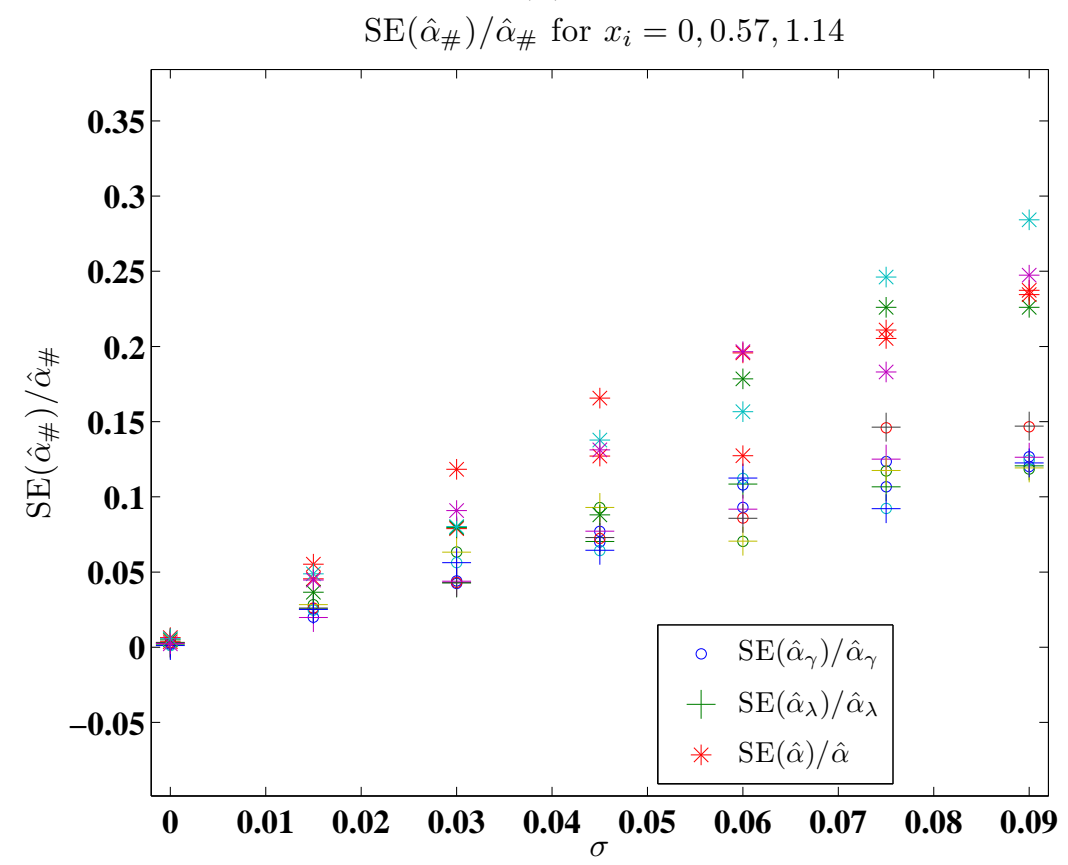

(b)

Figure 3.3: The results of realizations of simulated data with temporal nodes $t_{j}=$ $0.6+\frac{1}{120}, 0.6+\frac{2}{120}, \ldots, \frac{7}{120}, 20,40, \ldots, 140$ and spatial nodes $x_{i}=0,0.57,1.14$. The points denoted with ${ }^{o}$ are the ratio $\operatorname{SE}\left(\hat{\alpha}_{\gamma}\right) / \hat{\alpha}_{\gamma}$, the points denoted are the $\operatorname{SE}\left(\hat{\alpha}_{\lambda}\right) / \hat{\alpha}_{\lambda}$, the points denoted * are the difference $\operatorname{SE}(\hat{\alpha}) / \hat{\alpha}$ (a) The result of five realizations of $\mathbf{D}_{i j}(\sigma)$ for values of $\sigma=0.015,0.030, \ldots, 0.090$ (b) The result of five realizations of $\mathbf{D}_{i j}^{\text {rand }}(\sigma)$ for values of $\sigma=0,0.015, \ldots, 0.090$. 
based on our observation in (3.13) that $\gamma$ in the parameter estimation increases the error in the data.

The estimation of $\gamma$ is ill-posed for the parameter values, spatial nodes and temporal nodes in this problem. In Figures 3.6(a) and (b), we see that the difference $\hat{\gamma}-\gamma_{0}\left(\gamma_{0}=10^{-3}\right)$ is very large compared to $\gamma_{0}$. This is true for both the realizations of $\mathbf{D}_{i j}(\sigma)$ in Figure 3.6(a) and the realizations of $\mathbf{D}_{i j}^{\text {rand }}(\sigma)$ in Figure 3.6(b). Though the differences $\hat{\gamma}_{\lambda}-\gamma_{0}$ are smaller than $\hat{\gamma}-\gamma_{0}$ Figures 3.6(a) and (b), in Figures 3.7(a) and (c) we see that the uncertainty associated with the estimate $\hat{\gamma}_{\lambda}$ is ver large. In Figure 3.7(a), we see that for the realizations of $\mathbf{D}_{i j}(\sigma)$ the ratios $\mathrm{SE}\left(\hat{\gamma}_{\lambda}\right) / \hat{\gamma}_{\lambda}$ does not appear to grow exponentially with $\sigma$ but the values of $\operatorname{SE}\left(\hat{\gamma}_{\lambda}\right) / \hat{\gamma}_{\lambda}$ are too large to be confident of even the sign of $\hat{\gamma}_{\lambda}$. The use of $\mathbf{D}_{i j}^{\text {rand }}(\sigma)$ in the OLS parameter estimation procedure does appear to affect the uncertainty associated with the parameter estimate $\hat{\gamma}_{\lambda}$ as we see in the exponential growth of $\operatorname{SE}\left(\hat{\gamma}_{\lambda}\right) / \hat{\gamma}_{\lambda}$ with $\sigma$ for realizations of $\mathbf{D}_{i j}^{\text {rand }}(\sigma)$ in Figure $3.7(\mathrm{c})$. The ratio $\mathrm{SE}(\hat{\gamma}) / \hat{\gamma}$ varies on an exponential scale for both realizations of $\mathbf{D}_{i j}(\sigma)$ and $\mathbf{D}_{i j}^{\text {rand }}(\sigma)$ in Figures 3.7(b) and (d), respectively.

We also considered data at temporal nodes $t_{j}=0.6+\frac{1}{120}, 0.6+\frac{2}{120}, \ldots, 0.6+\frac{7}{120}, 20$, $40, \ldots, 140$ and spatial nodes $x_{i}=0,0.57$ to understand how sparsity of spatial data affects the inverse problem. For each level of added noise $\sigma=0.015,0.030, \ldots, 0.090$, we simulated five realizations of $\mathbf{D}_{i j}(\sigma)$ using (3.14). We also simulated five realizations of $\mathrm{d}_{i j}^{\text {rand }}(\sigma)$ using (3.15) for each level of added noise $\sigma=0,0.015, \ldots, 0.090$. For each realization of $\mathbf{D}_{i j}^{\text {rand }}(\sigma)$ a different random geometry $\Omega$ is used to solve (2.1) for $u_{i j}^{\text {rand }}$ in (3.16). Results from these simulations are given in Figures 3.8-3.13.

For the realizations of $\mathbf{D}_{i j}(\sigma)$, the differences $\hat{\alpha}_{\#}-\alpha_{0}$ are plotted in Figure 3.8(a) while these differences for the realizations of $\mathbf{D}_{i j}^{\text {rand }}(\sigma)$ are plotted in Figure 3.8(b). Much like these quantities in Figures 3.2(a) and (b), for each realization the differences $\hat{\alpha}_{\lambda}-\alpha_{0}, \hat{\alpha}_{\gamma}-\alpha_{0}$ and $\hat{\alpha}-\alpha_{0}$ remain relatively close and are on about the same scale as in Figures 3.2(a) and (b). The ratios $\operatorname{SE}\left(\hat{\alpha}_{\#}\right) / \hat{\alpha}_{\#}$ for the realizations of $\mathbf{D}_{i j}(\sigma)$ and $\mathbf{D}_{i j}^{\text {rand }}(\sigma)$ are plotted in Figures $3.9(\mathrm{a})$ and $(\mathrm{b})$, respectively. In both figures, it appears that the ratio $\mathrm{SE}(\hat{\alpha}) / \hat{\alpha}$ is larger than the ratios $\operatorname{SE}\left(\hat{\alpha}_{\lambda}\right) / \hat{\alpha}_{\lambda}$ and $\operatorname{SE}\left(\hat{\alpha}_{\gamma}\right) / \hat{\alpha}_{\gamma}$. It also appears that the relationship between the ratios $\operatorname{SE}\left(\hat{\alpha}_{\#}\right) / \hat{\alpha}_{\#}$ and $\sigma$ is linear as we observed in Figures 3.3(a) and (b) as well. This suggests that for the temporal and spatial nodes that we are considering estimating $\alpha$ is well-posed and there is little difference between using realizations of $\mathbf{D}_{i j}^{\text {rand }}(\sigma)$ and $\mathbf{D}_{i j}(\sigma)$ in the estimation of the parameter $\alpha$ and the estimation of the uncertainty associated with $\alpha$.

In Figures 3.10(a) and (b), we see that the difference $\hat{\lambda}-\lambda_{0}$ is larger than $\hat{\lambda}_{\gamma}-\lambda_{0}$ for the realizations of $\mathbf{D}_{i j}(\sigma)$ and $\mathbf{D}_{i j}^{\text {rand }}(\sigma)$, respectively. Figure 3.10(b) resembles Figure 3.10(a) which suggests that using realizations of $\mathbf{D}_{i j}^{\text {rand }}(\sigma)$ rather than realizations of $\mathbf{D}_{i j}(\sigma)$ does not have a substantial effect on the accuracy of the parameter estimates $\hat{\lambda}_{\#}$. The ratios $\operatorname{SE}\left(\hat{\lambda}_{\gamma}\right) / \hat{\lambda}_{\gamma}$ appear to be similar for realizations of $\mathbf{D}_{i j}(\sigma)$ (in Figure 3.11(a)) and realizations of $\mathbf{D}_{i j}^{\text {rand }}(\sigma)$ (in Figure 3.11(c)) and seem to vary linearly with $\sigma$. The ratios $\operatorname{SE}(\hat{\lambda}) / \hat{\lambda}$ are much larger than the ratios $\operatorname{SE}\left(\hat{\lambda}_{\gamma}\right) / \hat{\lambda}_{\gamma}$, so we plotted $\log (\operatorname{SE}(\hat{\lambda}) / \hat{\lambda})$ for the realizations of $\mathbf{D}_{i j}(\sigma)$ in Figure 3.11(b) and for the realizations of $\mathbf{D}_{i j}^{\text {rand }}(\sigma)$ in Figure 3.11(d). This implies 


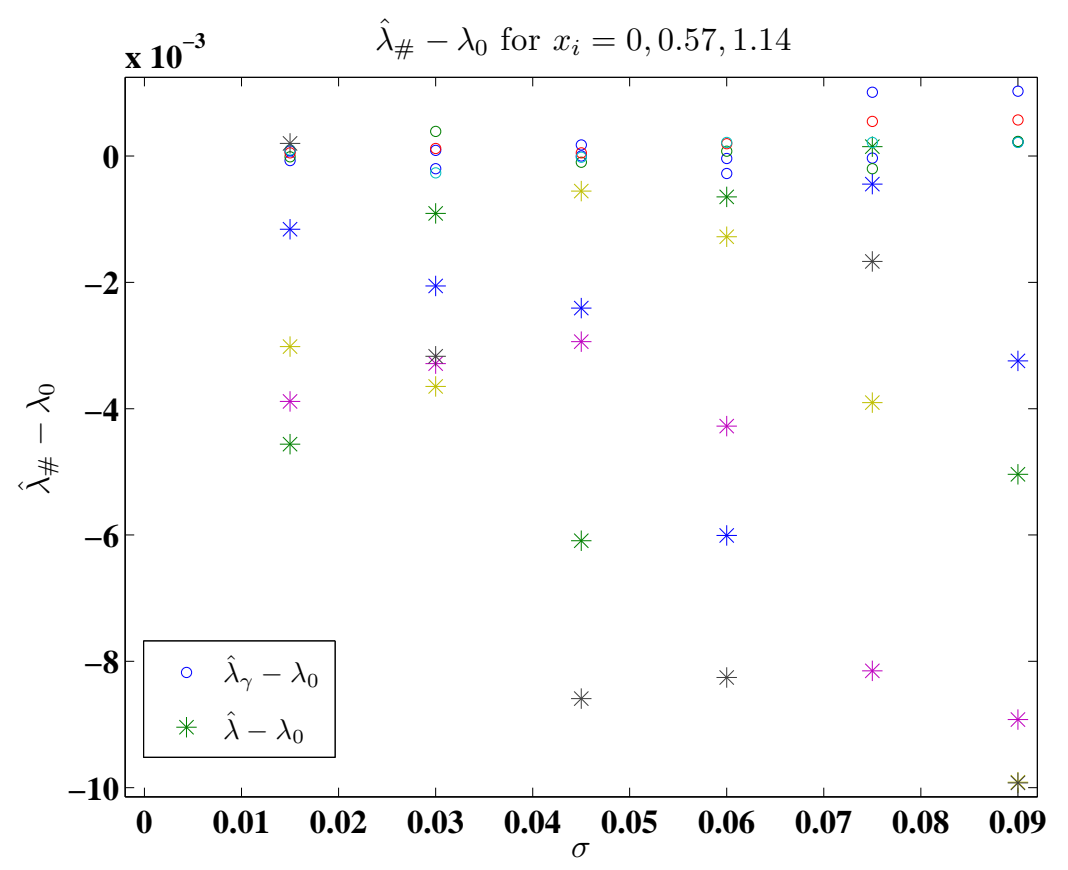

(a)

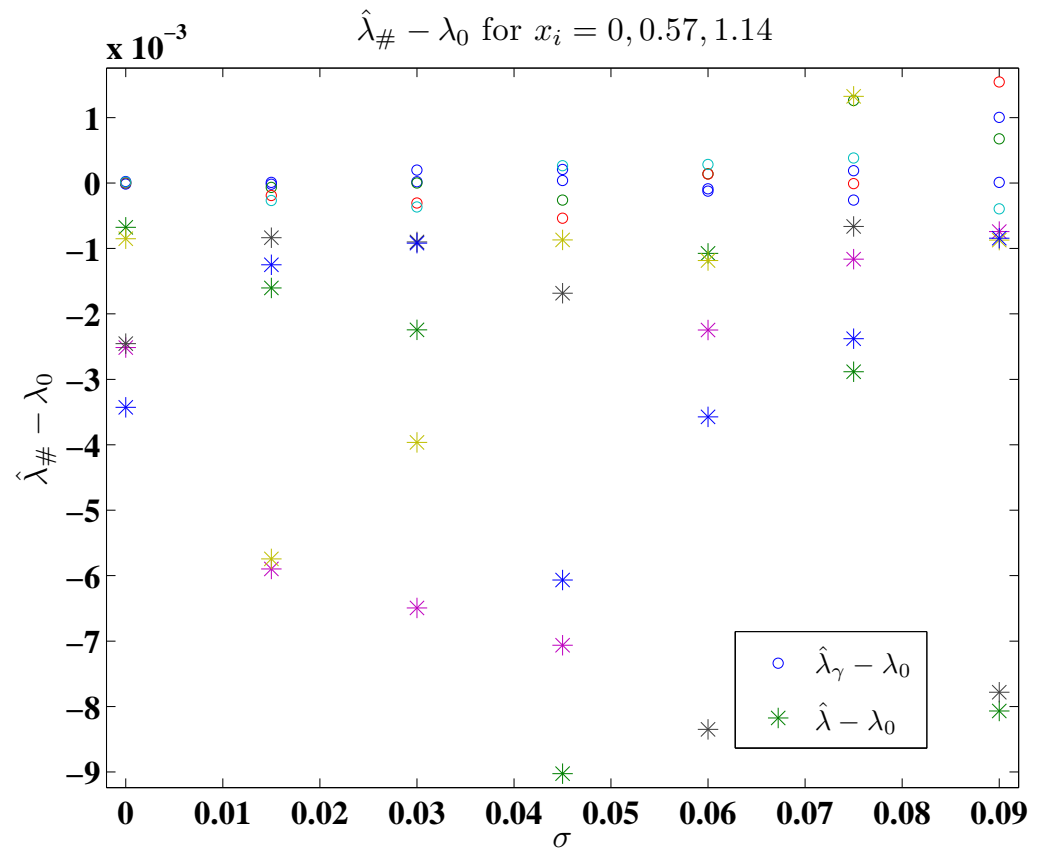

(b)

Figure 3.4: The results of realizations of simulated data with temporal nodes $t_{j}=0.6+$ $\frac{1}{120}, 0.6+\frac{2}{120}, \ldots, \frac{7}{120}, 20,40, \ldots, 140$ and spatial nodes $x_{i}=0,0.57,1.14$. The points denoted with ${ }^{o}$ are the difference $\hat{\lambda}_{\gamma}-\lambda_{0}$, and the points denoted ${ }^{*}$ are the difference $\hat{\lambda}-\lambda_{0}$ (a) The result of five realizations of $\mathbf{D}_{i j}(\sigma)$ for values of $\sigma=0.015,0.030, \ldots, 0.090$ (b) The result of five realizations of $\mathbf{D}_{i j}^{\text {rand }}(\sigma)$ for values of $\sigma=0,0.015, \ldots, 0.090$. 


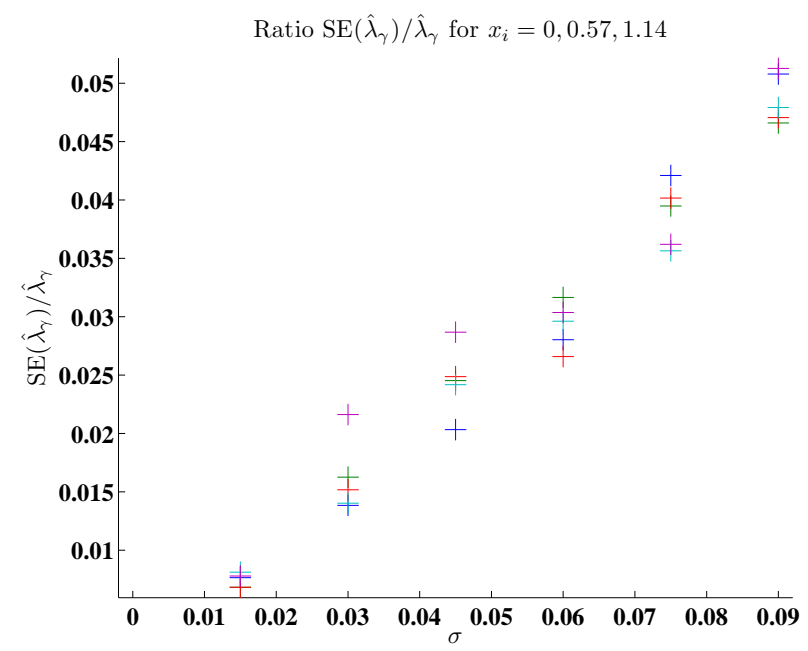

(a)

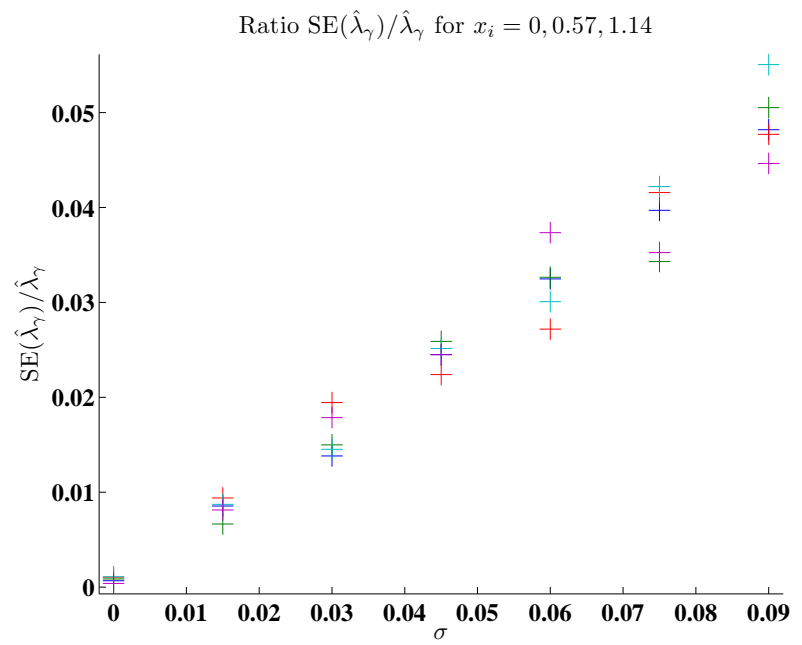

(c)

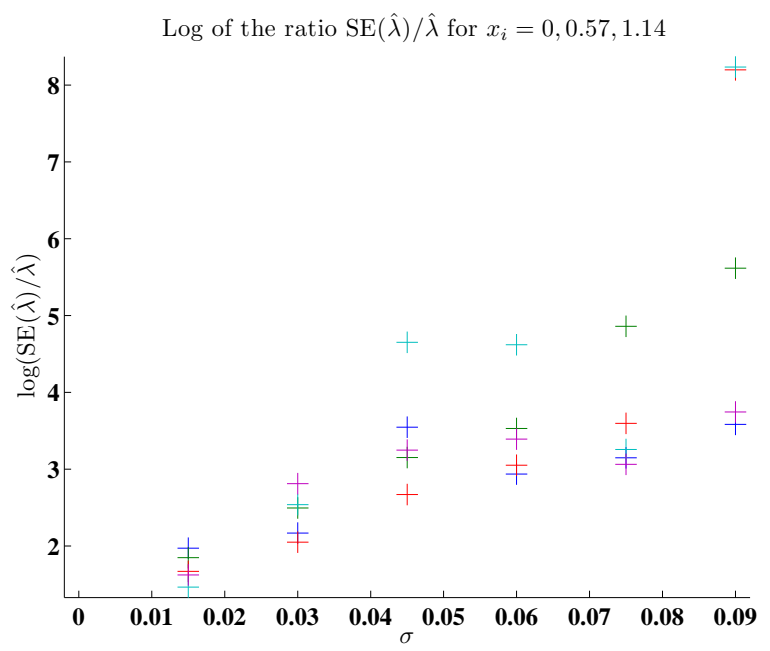

(b)

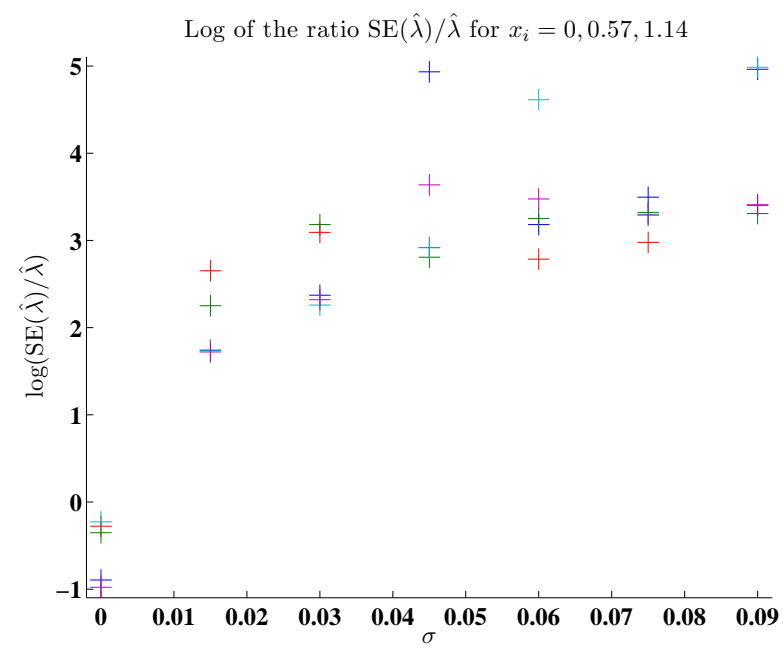

(d)

Figure 3.5: The results of realizations of simulated data with temporal nodes $t_{j}=$ $0.6+\frac{1}{120}, 0.6+\frac{2}{120}, \ldots, \frac{7}{120}, 20,40, \ldots, 140$ and spatial nodes $x_{i}=0,0.57,1.14$. (a) The ratio $\operatorname{SE}\left(\hat{\lambda}_{\gamma}\right) / \hat{\lambda}_{\gamma}$ for five realizations of $\mathbf{D}_{i j}(\sigma)$ for values of $\sigma=0.015,0.030, \ldots, 0.090$ (b) The $\log$ of the ratio $\log \operatorname{SE}(\hat{\lambda}) / \hat{\lambda}$ for five realizations of $\mathbf{D}_{i j}(\sigma)$ for values of $\sigma=0.015,0.030, \ldots, 0.090$. (c) The ratio $\operatorname{SE}\left(\hat{\lambda}_{\gamma}\right) / \hat{\lambda}_{\gamma}$ for five realizations of $\mathbf{D}_{i j}^{\text {rand }}(\sigma)$ for values of $\sigma=0,0.015, \ldots, 0.090$. (d) The $\log$ of the ratio $\log \mathrm{SE}(\hat{\lambda}) / \hat{\lambda}$ for five realizations of $\mathbf{D}_{i j}^{\text {rand }}(\sigma)$ for values of $\sigma=$ 0, 0.015, 0.030, . ., 0.090. 
$\hat{\gamma}_{\#}-\gamma_{0}$ for $x_{i}=0,0.57,1.14$

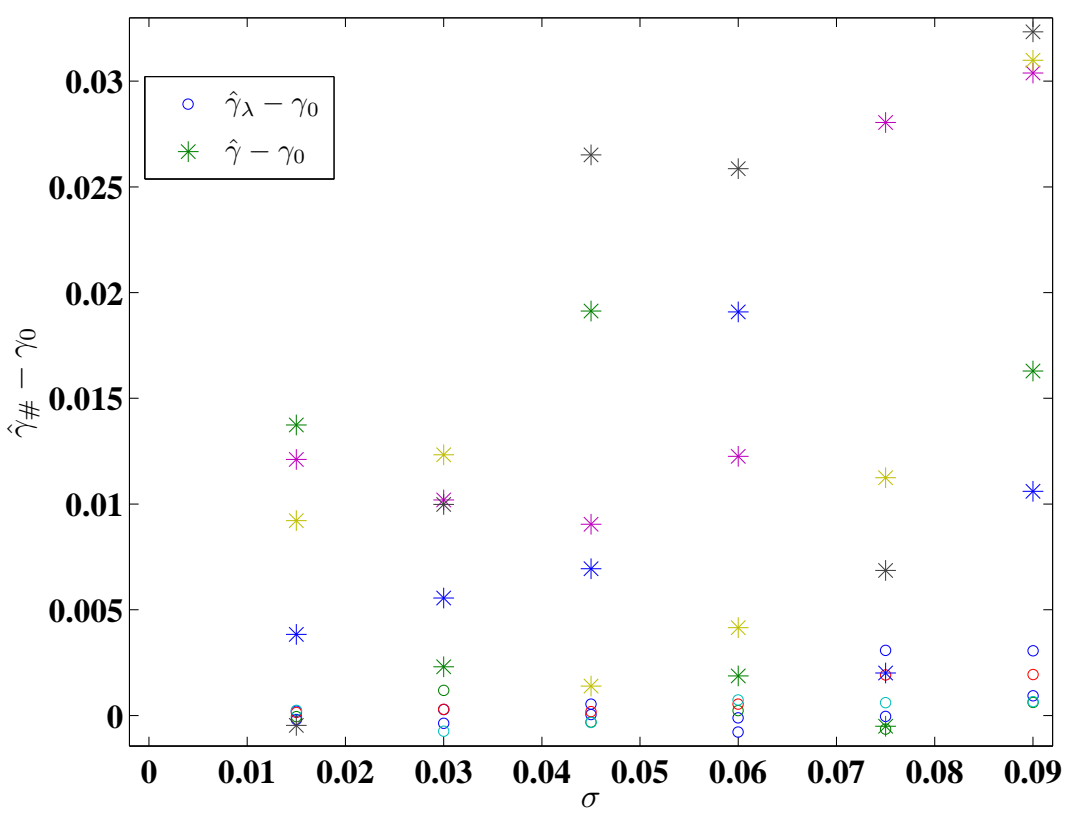

(a)

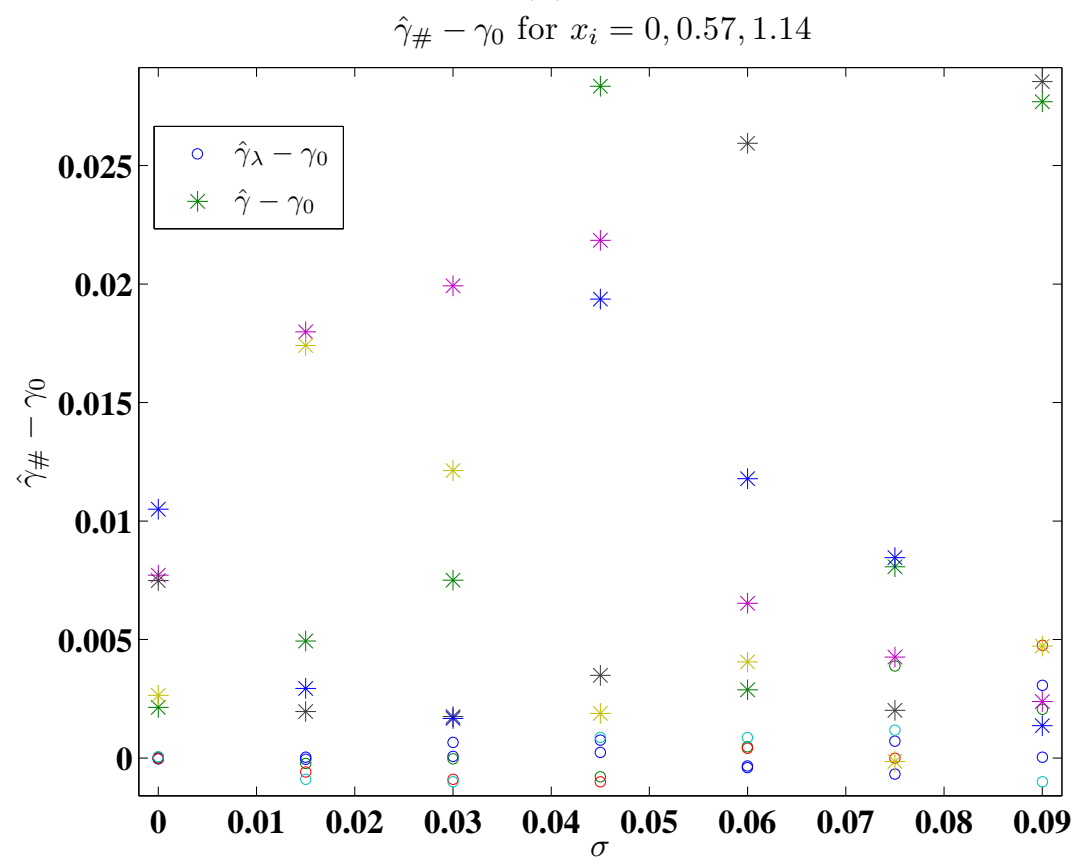

(b)

Figure 3.6: The results of realizations of simulated data with temporal nodes $t_{j}=0.6+$ $\frac{1}{120}, 0.6+\frac{2}{120}, \ldots, \frac{7}{120}, 20,40, \ldots, 140$ and spatial nodes $x_{i}=0,0.57,1.14$. The points denoted with ${ }^{o}$ are the difference $\hat{\gamma}_{\lambda}-\gamma_{0}$, and the points denoted ${ }^{*}$ are the difference $\hat{\gamma}-\gamma_{0}$ (a) The result of five realizations of $\mathbf{D}_{i j}(\sigma)$ for values of $\sigma=0.015,0.030, \ldots, 0.090$ (b) The result of five realizations of $\mathbf{D}_{i j}^{\text {rand }}(\sigma)$ for values of $\sigma=0,0.015, \ldots, 0.090$. 


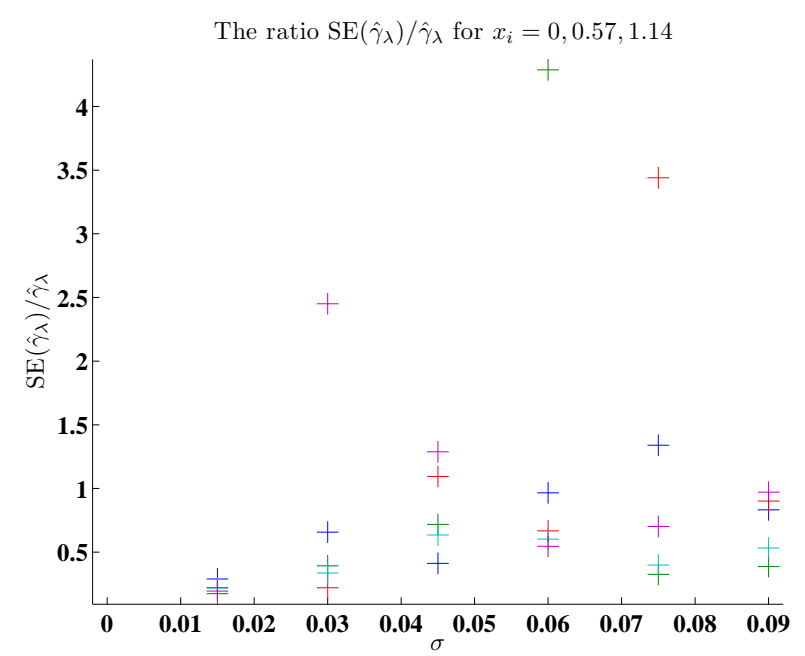

(a)

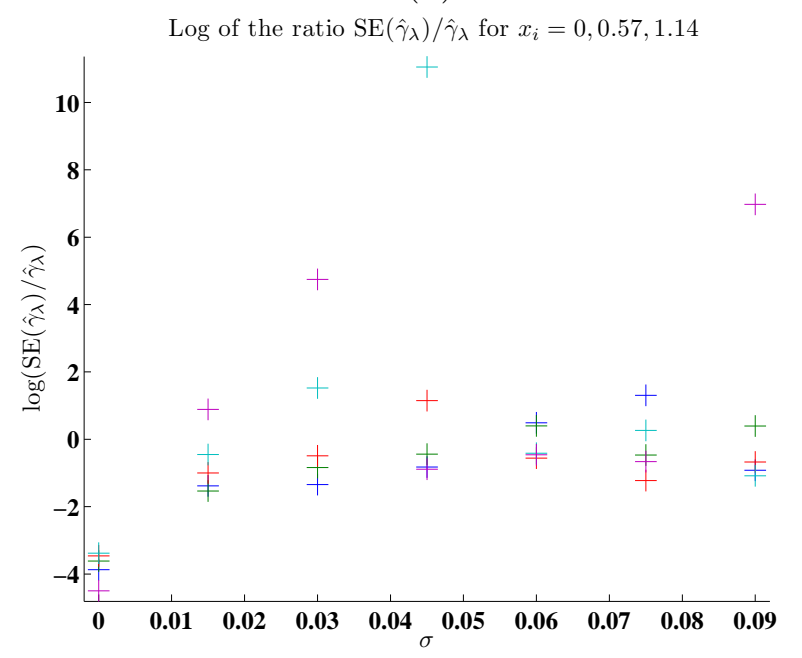

(c)

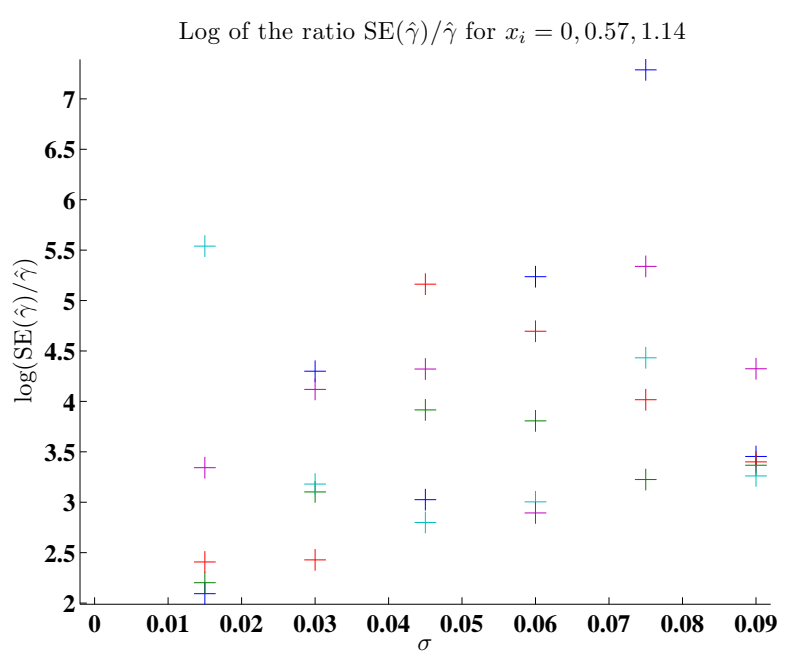

(b)

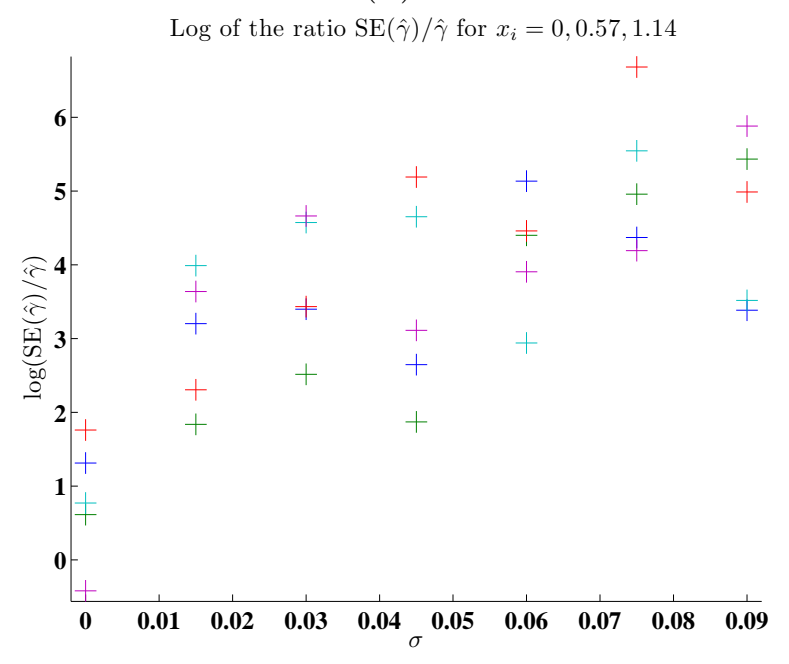

(d)

Figure 3.7: The results of realizations of simulated data with temporal nodes $t_{j}=$ $0.6+\frac{1}{120}, 0.6+\frac{2}{120}, \ldots, \frac{7}{120}, 20,40, \ldots, 140$ and spatial nodes $x_{i}=0,0.57,1.14$. (a) The ratio $\operatorname{SE}\left(\hat{\gamma}_{\lambda}\right) / \hat{\gamma}_{\lambda}$ for five realizations of $\mathbf{D}_{i j}(\sigma)$ for values of $\sigma=0.015,0.030, \ldots, 0.090$ (b) The log of the ratio $\log \mathrm{SE}(\hat{\gamma}) / \hat{\gamma}$ for five realizations of $\mathbf{D}_{i j}(\sigma)$ for values of $\sigma=0.015,0.030, \ldots, 0.090$. (c) The $\log$ of the ratio $\log \left(\mathrm{SE}\left(\hat{\gamma}_{\lambda}\right) / \hat{\gamma}_{\lambda}\right)$ for five realizations of $\mathbf{D}_{i j}^{\text {rand }}(\sigma)$ for values of $\sigma=0,0.015, \ldots, 0.090$. (d) The $\log$ of the ratio $\log \mathrm{SE}(\hat{\gamma}) / \hat{\gamma}$ for five realizations of $\mathbf{D}_{i j}^{\text {rand }}(\sigma)$ for values of $\sigma=0,0.015,0.030, \ldots, 0.090$. 


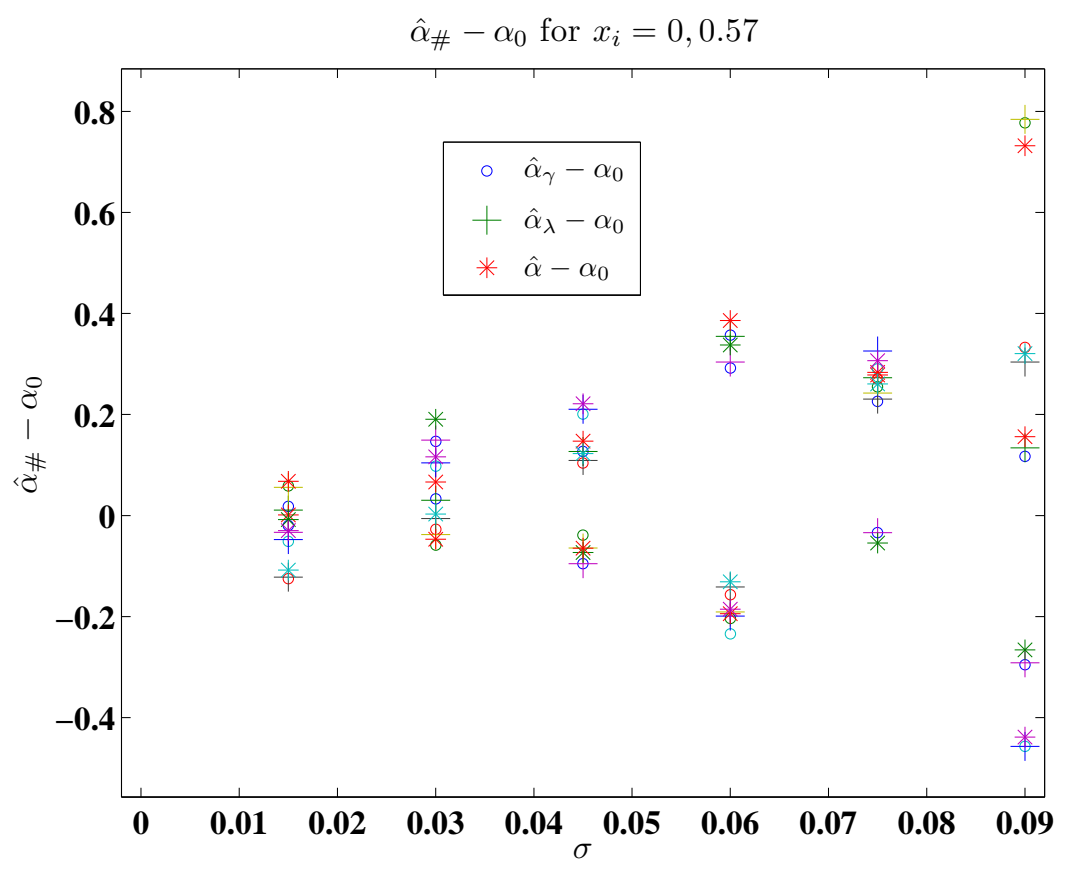

(a)

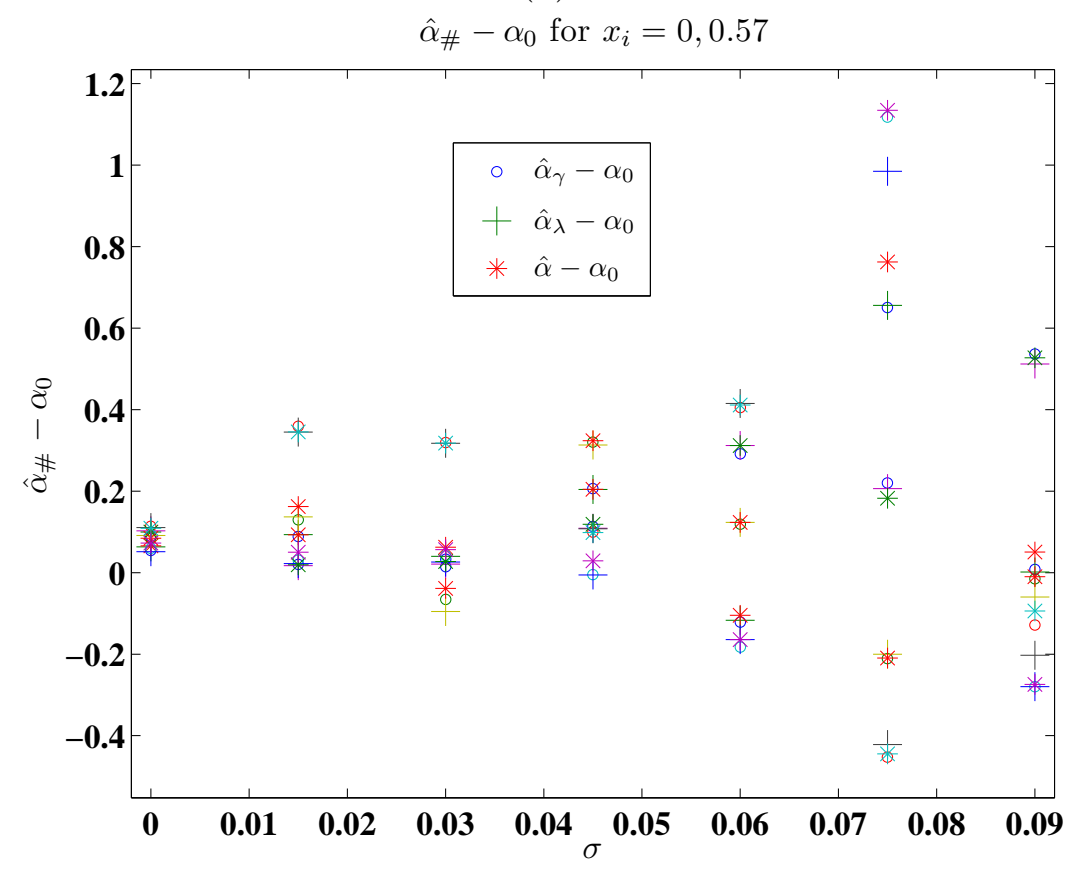

(b)

Figure 3.8: The results of realizations of simulated data with temporal nodes $t_{j}=$ $0.6+\frac{1}{120}, 0.6+\frac{2}{120}, \ldots, \frac{7}{120}, 20,40, \ldots, 140$ and spatial nodes $x_{i}=0,0.57$. The points denoted with ${ }^{o}$ are the difference $\hat{\alpha}_{\gamma}-\alpha_{0}$, the points denoted are the $\hat{\alpha}_{\lambda}-\alpha_{0}$, the points denoted * are the difference $\hat{\alpha}-\alpha_{0}$ (a) The result of five realizations of $\mathbf{D}_{i j}(\sigma)$ for values of $\sigma=0.015,0.030, \ldots, 0.090$ (b) The result of five realizations of $\mathbf{D}_{i j}^{\text {rand }}(\sigma)$ for values of $\sigma=0,0.015, \ldots, 0.090$. 
$\operatorname{SE}\left(\hat{\alpha}_{\#}\right) / \hat{\alpha}_{\#}$ for $x_{i}=0,0.57$

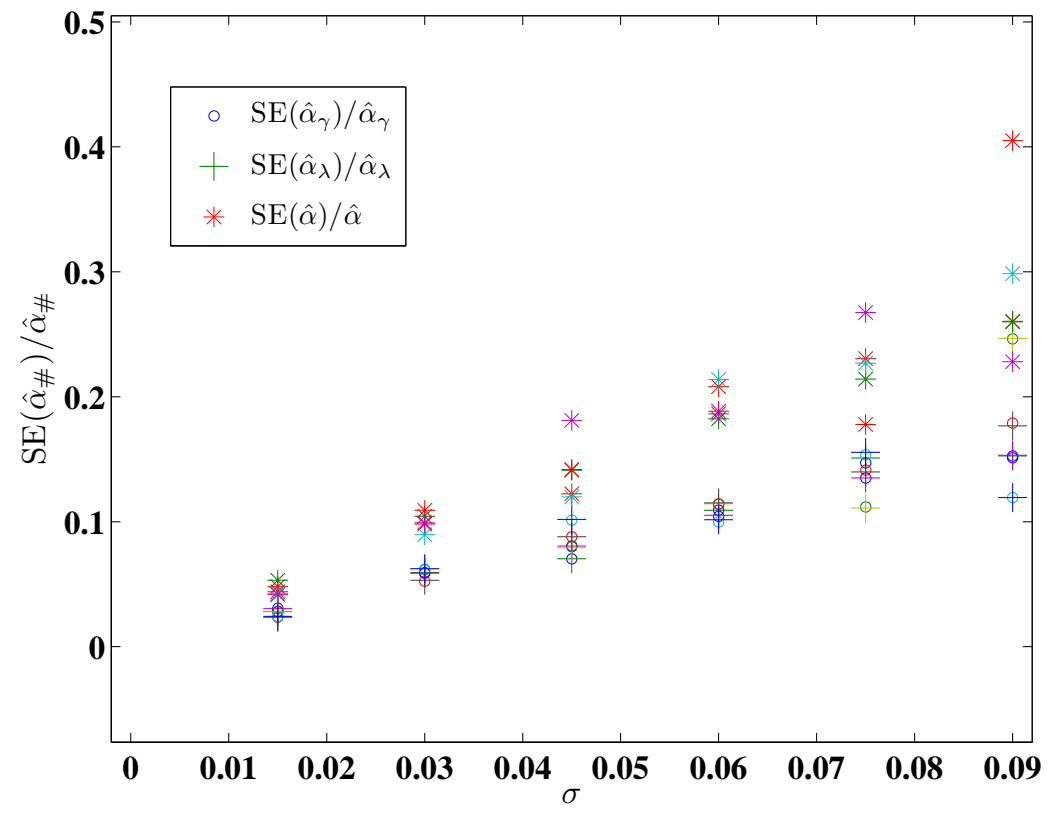

(a)

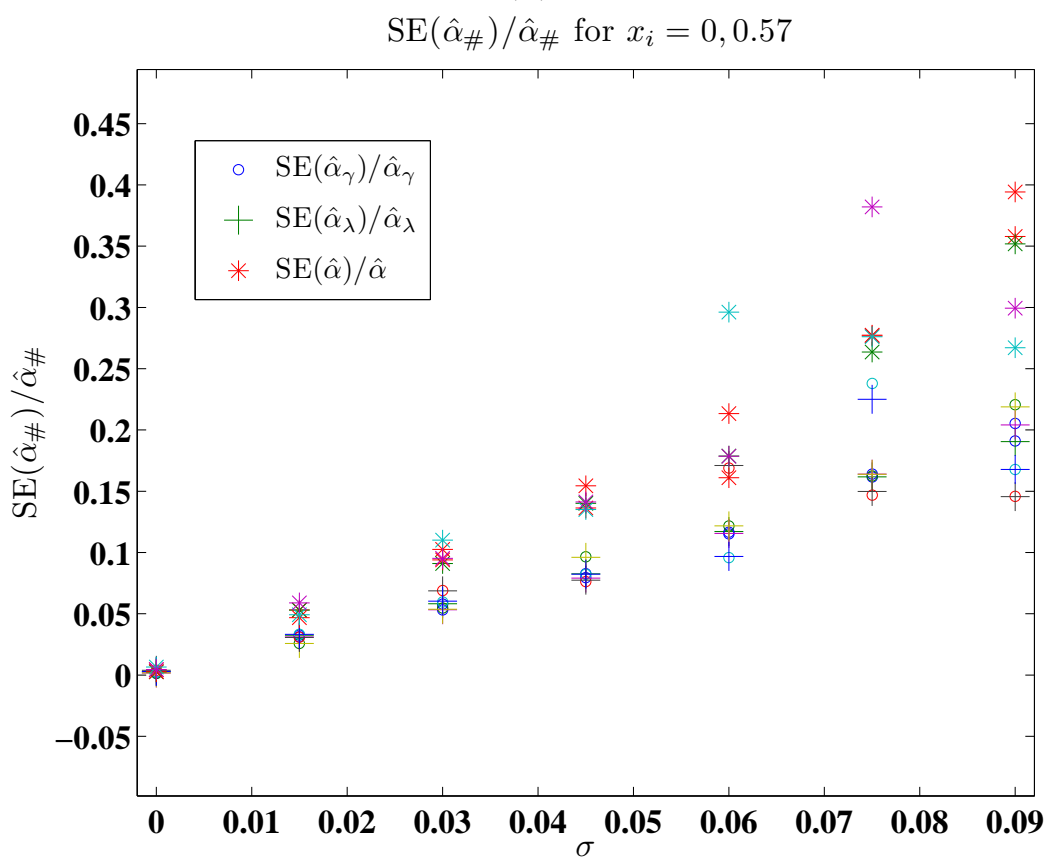

(b)

Figure 3.9: The results of realizations of simulated data with temporal nodes $t_{j}=$ $0.6+\frac{1}{120}, 0.6+\frac{2}{120}, \ldots, \frac{7}{120}, 20,40, \ldots, 140$ and spatial nodes $x_{i}=0,0.57$. The points denoted with ${ }^{o}$ are the ratio $\operatorname{SE}\left(\hat{\alpha}_{\gamma}\right) / \hat{\alpha}_{\gamma}$, the points denoted are the $\operatorname{SE}\left(\hat{\alpha}_{\lambda}\right) / \hat{\alpha}_{\lambda}$, the points denoted * are the difference $\operatorname{SE}(\hat{\alpha}) / \hat{\alpha}$ (a) The result of five realizations of $\mathbf{D}_{i j}(\sigma)$ for values of $\sigma=0.015,0.030, \ldots, 0.090$ (b) The result of five realizations of $\mathbf{D}_{i j}^{\text {rand }}(\sigma)$ for values of $\sigma=0,0.015, \ldots, 0.090$. 
that for realizations of $\mathbf{D}_{i j}(\sigma)$ and $\mathbf{D}_{i j}^{\text {rand }}(\sigma)$ estimating $\gamma$ causes a dramatic increase in the uncertainty associated with the parameter estimate $\hat{\lambda}$ for spatial nodes $x_{i}=0,0.57$. Note that this effect appears to be similar for realizations of $\mathbf{D}_{i j}(\sigma)$ in Figure $3.11(\mathrm{~b})$ as for realizations of $\mathbf{D}_{i j}^{\text {rand }}(\sigma)$ in Figure $3.11(\mathrm{~d})$.

The estimation of the parameter $\gamma$ appears to be ill-posed for the spatial nodes $x_{i}=$ $0,0.57$, especially when estimating the parameter set $(\gamma, \alpha, \lambda)$. The differences $\hat{\gamma}-\gamma_{0}$ are several orders of magnitude larger than the "true" parameter $\gamma_{0}=10^{-3}$ in Figure 3.12(a) (for realizations of $\mathbf{D}_{i j}(\sigma)$ ) and in Figure 3.6(b) (for realizations of $\mathbf{D}_{i j}^{\text {rand }}(\sigma)$ ).

For the spatial nodes $x_{i}=0,0.57$, the uncertainty associated with the parameter estimate $\hat{\gamma}_{\#}$ is very large. For all of the examples of $\mathrm{SE}\left(\hat{\gamma}_{\#}\right) / \hat{\gamma}_{\#}$ in Figures 3.13(a)-(d) we plotted $\log \left(\operatorname{SE}\left(\hat{\gamma}_{\#}\right) / \hat{\gamma}_{\#}\right)$ because the variation of $\mathrm{SE}\left(\hat{\gamma}_{\#}\right) / \hat{\gamma}_{\#}$ was so large for every example. Figures 3.13(a) and (c) depict $\mathrm{SE}\left(\hat{\gamma}_{\lambda}\right) / \hat{\gamma}_{\lambda}$ for the realizations of $\mathbf{D}_{i j}(\sigma)$ and $\mathbf{D}_{i j}^{\text {rand }}(\sigma)$, respectively. The ratios $\mathrm{SE}\left(\hat{\gamma}_{\lambda}\right) / \hat{\gamma}_{\lambda}$ are on an exponential scale for realizations of $\mathbf{D}_{i j}(\sigma)$ in Figure 3.13(a) with spatial nodes $x_{i}=0,0.57$ while the ratios $\mathrm{SE}\left(\hat{\gamma}_{\lambda}\right) / \hat{\gamma}_{\lambda}$ are on a linear scale for realizations of $\mathbf{D}_{i j}(\sigma)$ in Figure 3.7(a) which indicates that sparsity of spatial collection nodes affects the uncertainty associated with the parameter estimates $\hat{\gamma}_{\#}$. 


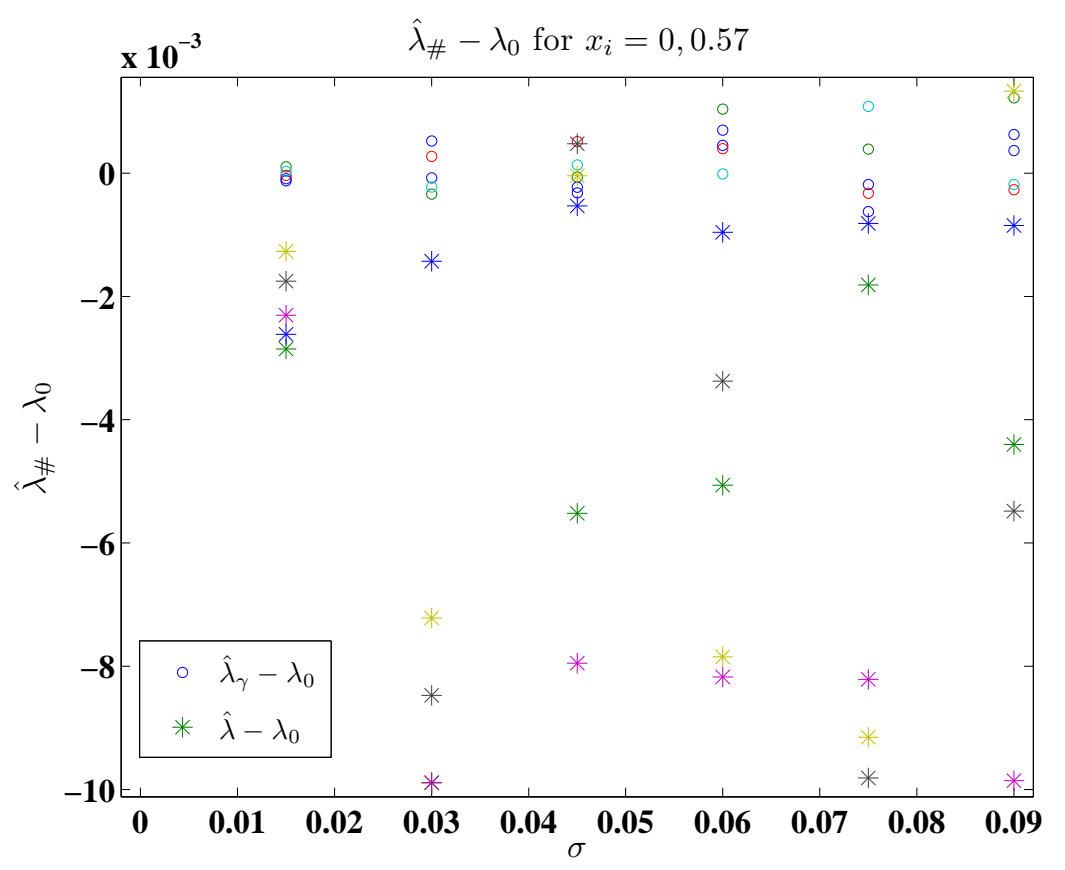

(a)

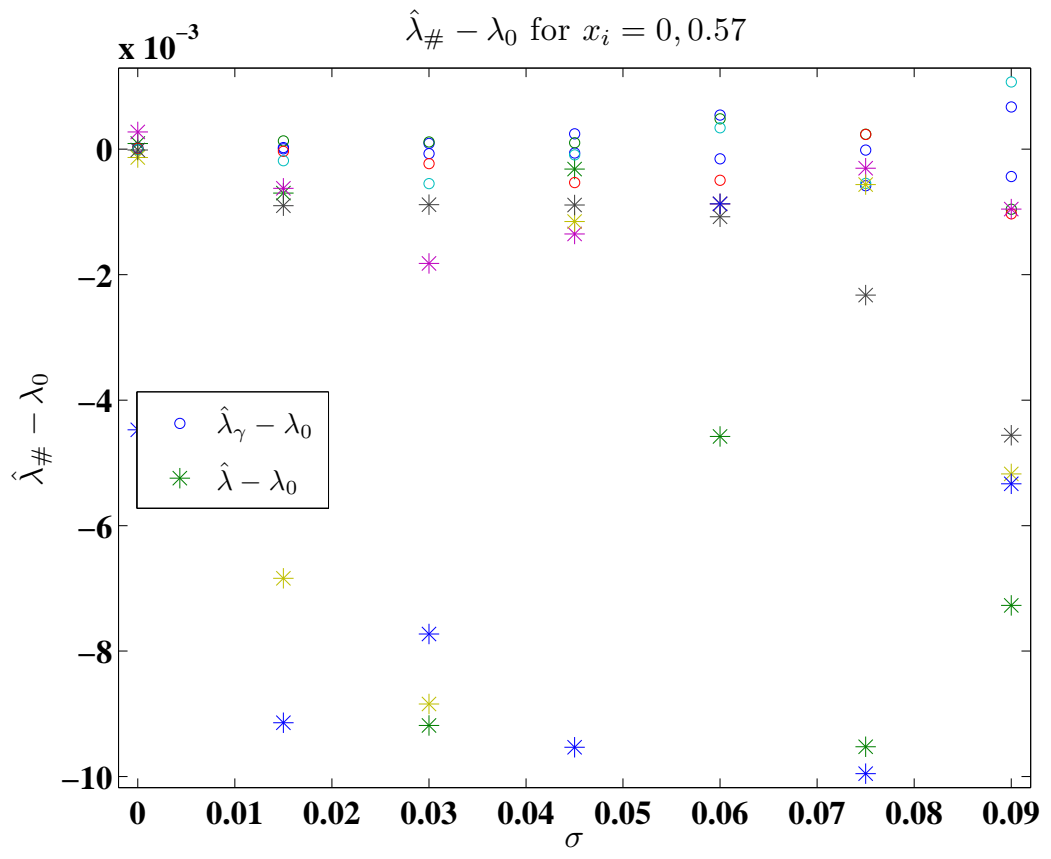

(b)

Figure 3.10: The results of realizations of simulated data with temporal nodes $t_{j}=0.6+$ $\frac{1}{120}, 0.6+\frac{2}{120}, \ldots, \frac{7}{120}, 20,40, \ldots, 140$ and spatial nodes $x_{i}=0,0.57$. The points denoted with ${ }^{o}$ are the difference $\hat{\lambda}_{\gamma}-\lambda_{0}$, and the points denoted ${ }^{*}$ are the difference $\hat{\lambda}-\lambda_{0}$ (a) The result of five realizations of $\mathbf{D}_{i j}(\sigma)$ for values of $\sigma=0.015,0.030, \ldots, 0.090$ (b) The result of five realizations of $\mathbf{D}_{i j}^{\text {rand }}(\sigma)$ for values of $\sigma=0,0.015, \ldots, 0.090$. 


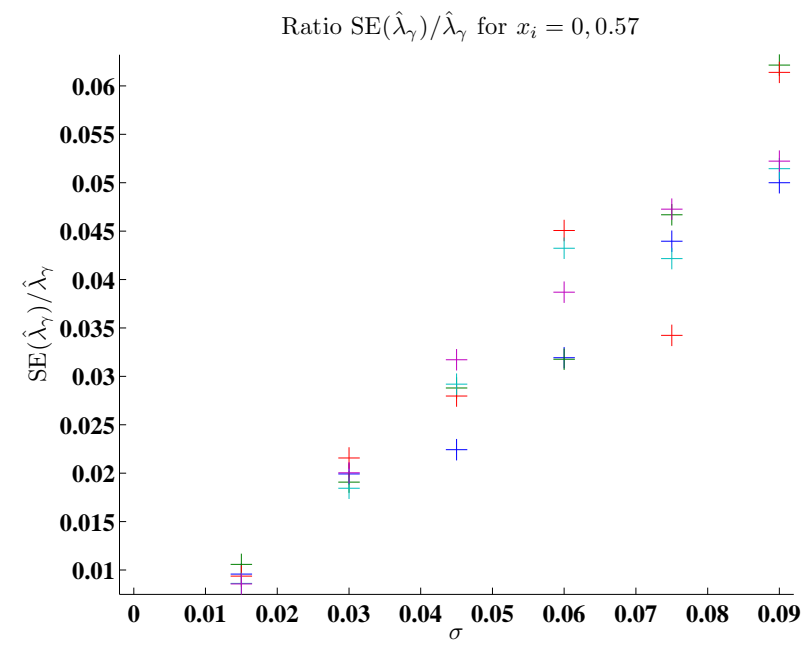

(a)

Ratio $\operatorname{SE}\left(\hat{\lambda}_{\gamma}\right) / \hat{\lambda}_{\gamma}$ for $x_{i}=0,0.57$

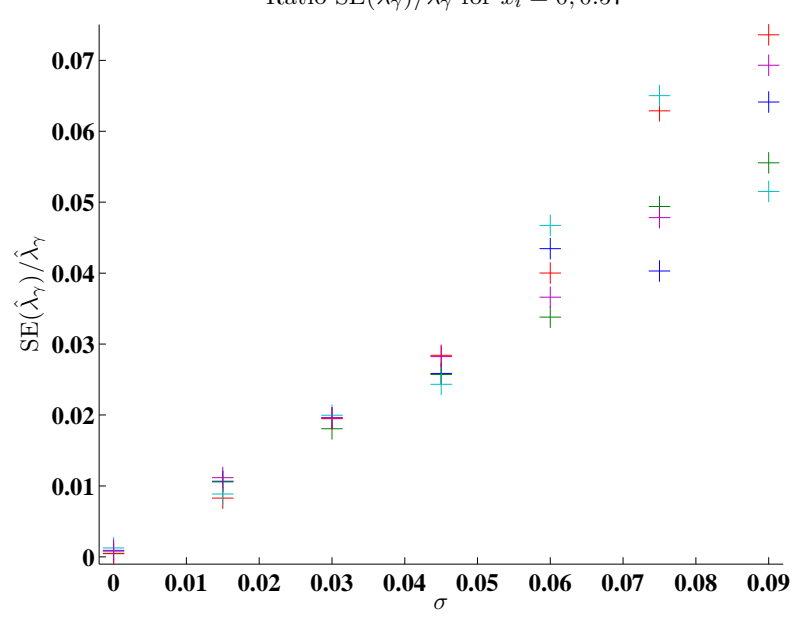

(c)

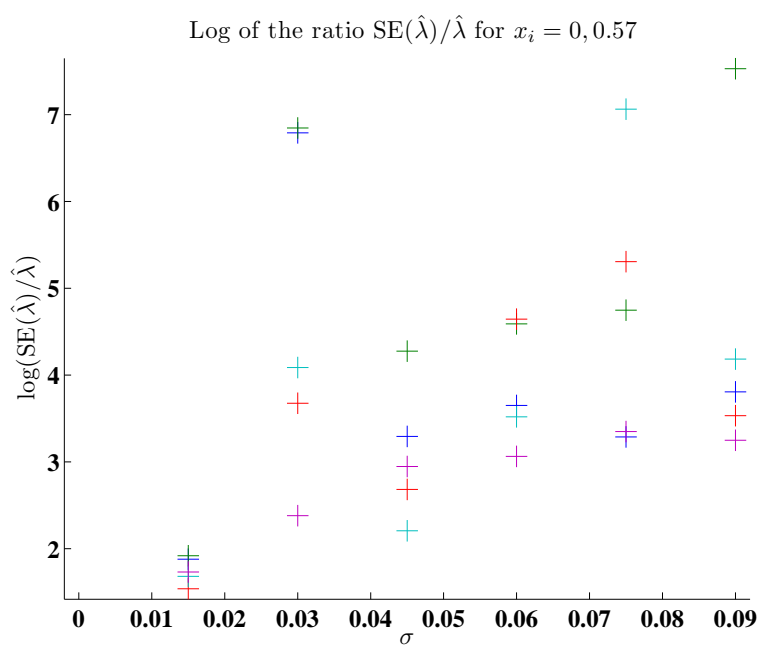

(b)

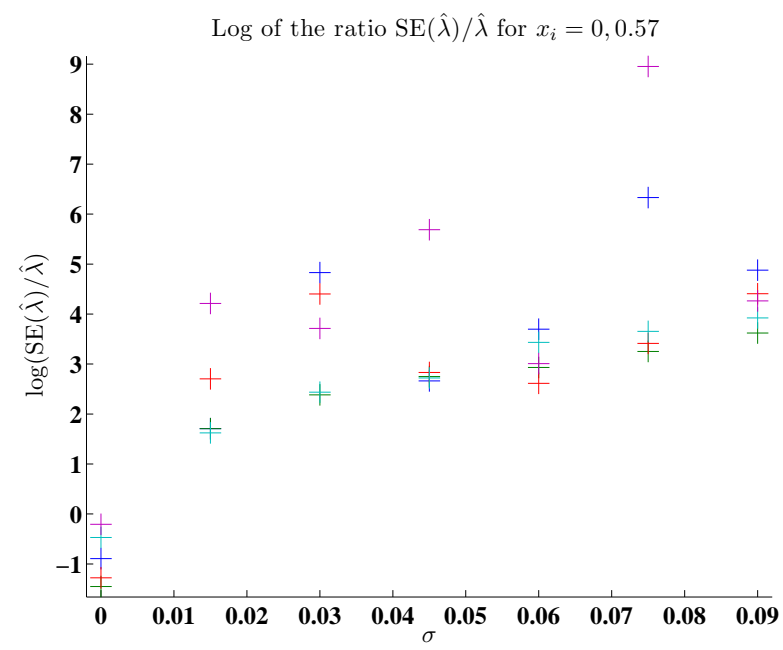

(d)

Figure 3.11: The results of realizations of simulated data with temporal nodes $t_{j}=0.6+$ $\frac{1}{120}, 0.6+\frac{2}{120}, \ldots, \frac{7}{120}, 20,40, \ldots, 140$ and spatial nodes $x_{i}=0,0.57$. (a) The ratio $\operatorname{SE}\left(\hat{\lambda}_{\gamma}\right) / \hat{\lambda}_{\gamma}$ for five realizations of $\mathbf{D}_{i j}(\sigma)$ for values of $\sigma=0.015,0.030, \ldots, 0.090$ (b) The $\log$ of the ratio $\log \operatorname{SE}(\hat{\lambda}) / \hat{\lambda}$ for five realizations of $\mathbf{D}_{i j}(\sigma)$ for values of $\sigma=0.015,0.030, \ldots, 0.090$. (c) The ratio $\operatorname{SE}\left(\hat{\lambda}_{\gamma}\right) / \hat{\lambda}_{\gamma}$ for five realizations of $\mathbf{D}_{i j}^{\text {rand }}(\sigma)$ for values of $\sigma=0,0.015, \ldots, 0.090$. (d) The $\log$ of the ratio $\log \operatorname{SE}(\hat{\lambda}) / \hat{\lambda}$ for five realizations of $\mathbf{D}_{i j}^{\text {rand }}(\sigma)$ for values of $\sigma=$ $0,0.015,0.030, \ldots, 0.090$. 


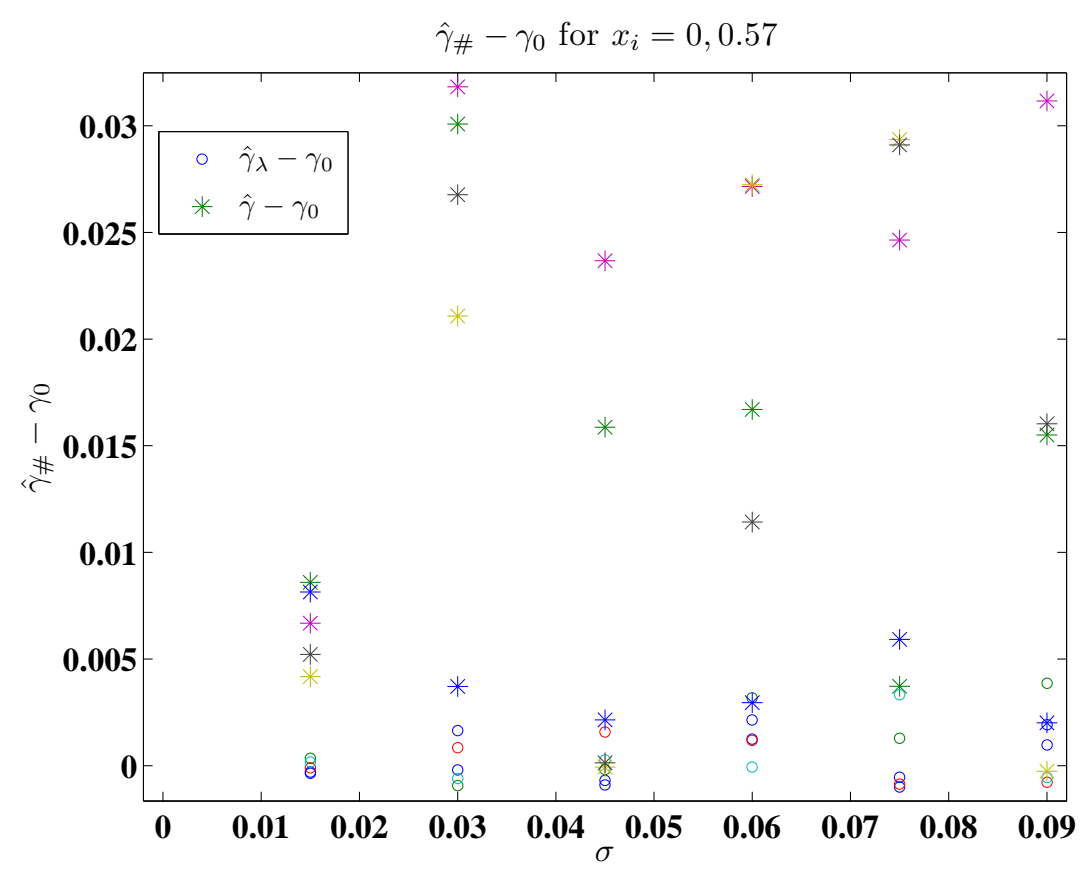

(a)

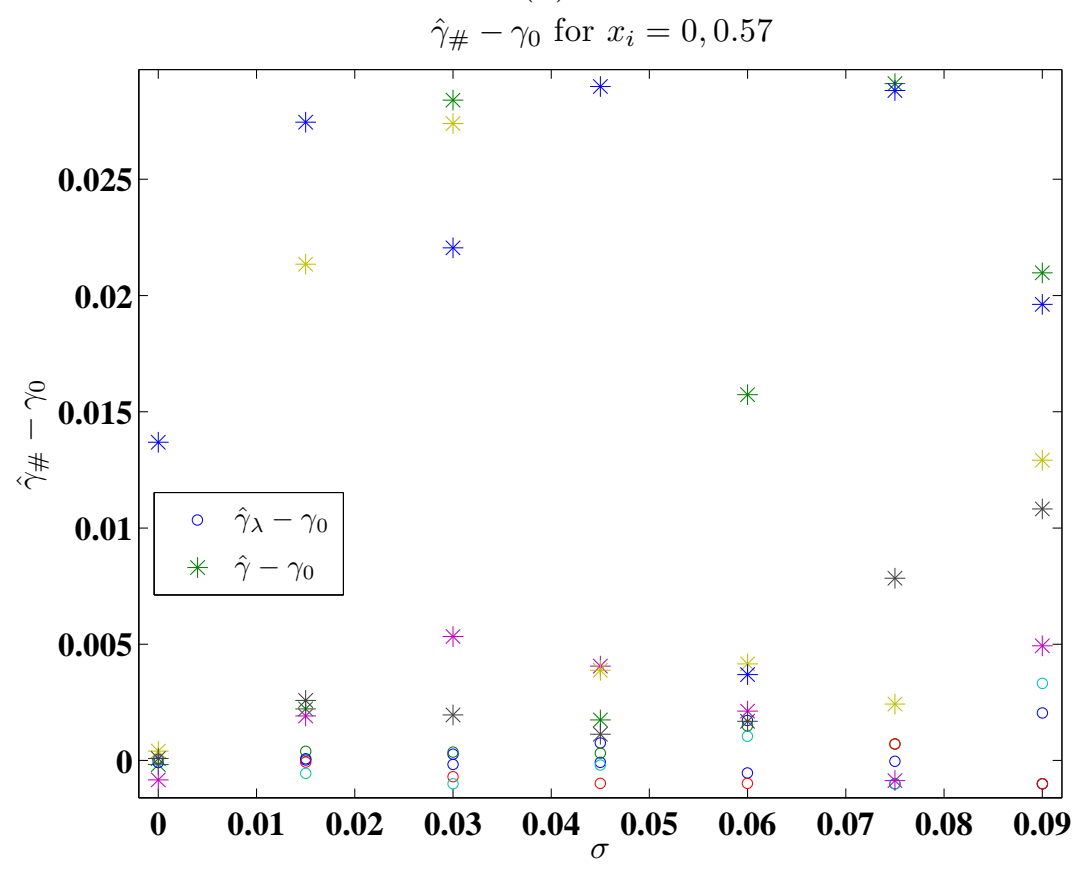

(b)

Figure 3.12: The results of realizations of simulated data with temporal nodes $t_{j}=0.6+$ $\frac{1}{120}, 0.6+\frac{2}{120}, \ldots, \frac{7}{120}, 20,40, \ldots, 140$ and spatial nodes $x_{i}=0,0.57$. The points denoted with ${ }^{\circ}$ are the difference $\hat{\gamma}_{\lambda}-\gamma_{0}$, and the points denoted ${ }^{*}$ are the difference $\hat{\gamma}-\gamma_{0}$ (a) The result of five realizations of $\mathbf{D}_{i j}(\sigma)$ for values of $\sigma=0.015,0.030, \ldots, 0.090$ (b) The result of five realizations of $\mathbf{D}_{i j}^{\text {rand }}(\sigma)$ for values of $\sigma=0,0.015, \ldots, 0.090$. 


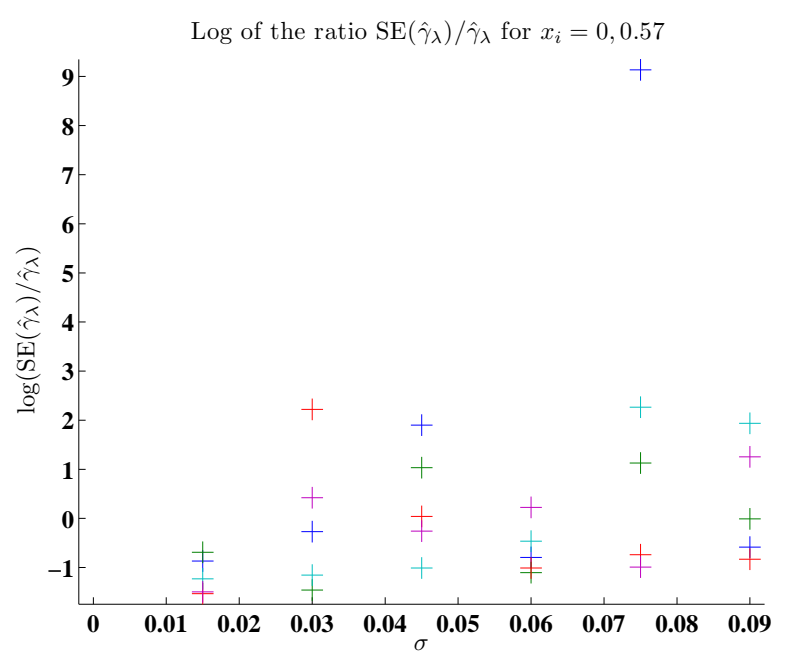

(a)

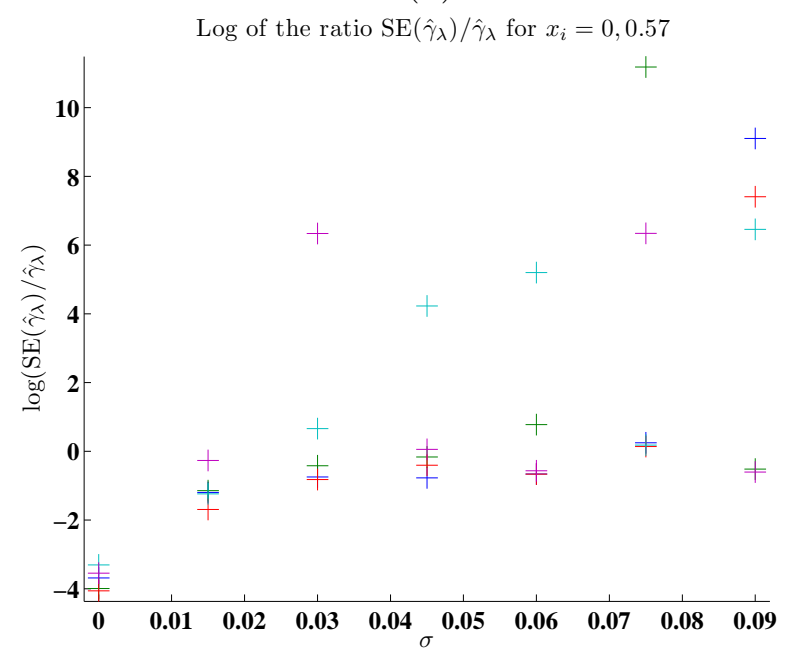

(c)

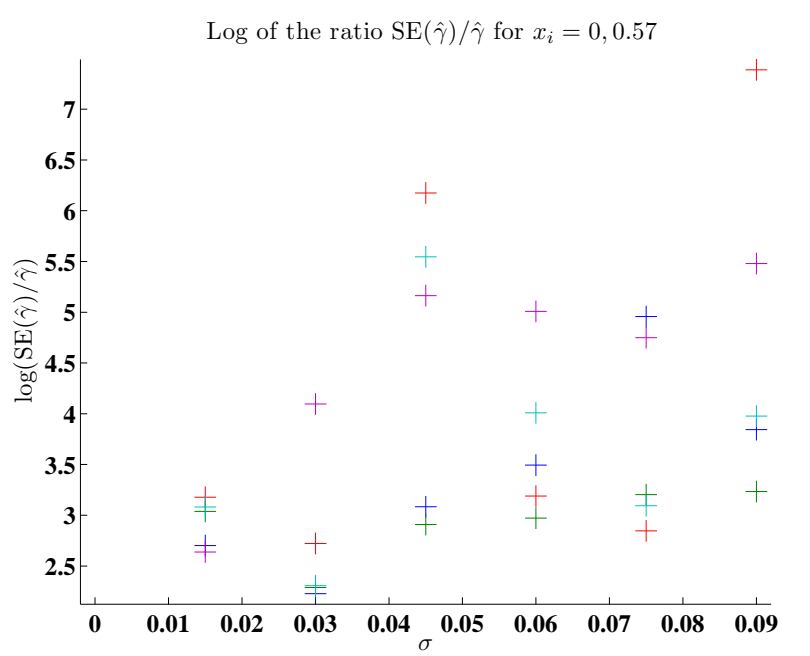

(b)

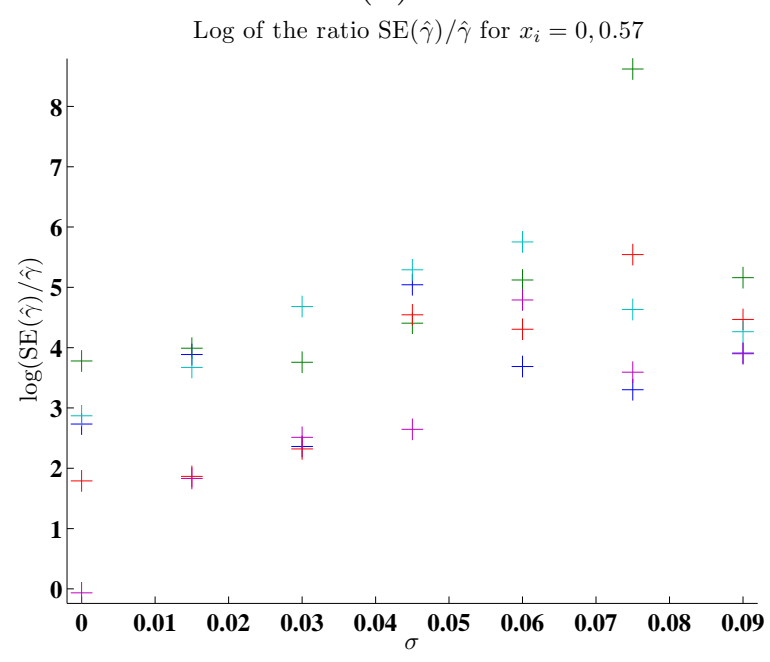

(d)

Figure 3.13: The results of realizations of simulated data with temporal nodes $t_{j}=0.6+$ $\frac{1}{120}, 0.6+\frac{2}{120}, \ldots, \frac{7}{120}, 20,40, \ldots, 140$ and spatial nodes $x_{i}=0,0.57$. (a) The ratio $\operatorname{SE}\left(\hat{\gamma}_{\lambda}\right) / \hat{\gamma}_{\lambda}$ for five realizations of $\mathbf{D}_{i j}(\sigma)$ for values of $\sigma=0.015,0.030, \ldots, 0.090$ (b) The log of the ratio $\log \mathrm{SE}(\hat{\gamma}) / \hat{\gamma}$ for five realizations of $\mathbf{D}_{i j}(\sigma)$ for values of $\sigma=0.015,0.030, \ldots, 0.090$. (c) The $\log$ of the ratio $\log \left(\operatorname{SE}\left(\hat{\gamma}_{\lambda}\right) / \hat{\gamma}_{\lambda}\right)$ for five realizations of $\mathbf{D}_{i j}^{\text {rand }}(\sigma)$ for values of $\sigma=$ $0,0.015, \ldots, 0.090$. (d) The $\log$ of the ratio $\log \mathrm{SE}(\hat{\gamma}) / \hat{\gamma}$ for five realizations of $\mathbf{D}_{i j}^{\text {rand }}(\sigma)$ for values of $\sigma=0,0.015,0.030, \ldots, 0.090$. 


\section{Generalized Least Squares}

The generalized least squares (GLS) parameter estimation procedure like the OLS parameter estimation procedure is based on an underlying statistical model. The error for GLS is assumed to be relative or proportional to the model value. Observations are assumed to be realizations of the random process $\boldsymbol{Y}_{i j}$ given by

$$
\boldsymbol{Y}_{i j}=U_{i j}\left(\theta_{0}^{\#}\right)\left(1+\mathcal{E}_{i j}\right)
$$

where $\mathcal{E}_{i j}$ is assumed to have constant variance, zero mean, and mutually independent or

$$
\begin{array}{ll}
E\left(\mathcal{E}_{i j}\right) & =0 \\
\operatorname{Var}\left(\mathcal{E}_{i j}\right) & =\sigma_{0}^{2} \\
\operatorname{Cov}\left(\mathcal{E}_{i j}, \mathcal{E}_{k h}\right) & =0 \text { for }(i, j) \neq(k, h) .
\end{array}
$$

Note that in $(4.1)$, the error is given by $U_{i j}\left(\theta_{0}^{\#}\right) \mathcal{E}_{i j}$ so it is proportional to the model and the variance is proportional to $U_{i j}^{2}\left(\theta_{0}^{\#}\right)$. Assuming the statistical model given by $(4.1)$, the GLS parameter estimation procedure involves the minimization of the cost functional

$$
J\left(\theta^{\#}\right)=\sum_{i=1}^{m} \sum_{j=1}^{n}\left(\frac{U_{i j}\left(\theta^{\#}\right)-y_{i j}}{U_{i j}\left(\theta^{\#}\right)}\right)^{2}
$$

The GLS parameter estimate is given by

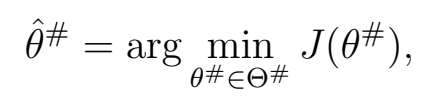

where $J\left(\theta^{\#}\right)$ is defined in (4.3). We used the iteratively reweighted least squares method as described in [6] and [13] to minimize (4.3). The GLS variance estimate $\hat{\sigma}_{\#}^{2}$ is given by

$$
\hat{\sigma}_{\#}^{2}=\frac{J\left(\hat{\theta}^{\#}\right)}{n m-p},
$$

where again $J\left(\hat{\theta}^{\#}\right)$ is defined in (4.3). The $n m \times n m$ matrix of weights $W\left(\theta^{\#}\right)$ has entries

$$
w_{i+m(j-1), i+m(j-1)}\left(\theta^{\#}\right)=\frac{1}{U_{i j}^{2}\left(\theta^{\#}\right)}
$$

for $i=1,2, \ldots, m$ and $j=1,2, \ldots, n$. The GLS covariance matrix estimate $\Sigma\left(\hat{\theta}^{\#}\right)$ is given [6] by

$$
\Sigma\left(\hat{\theta}^{\#}\right)=\hat{\sigma}^{2}\left(\chi^{T}\left(\hat{\theta}^{\#}\right) W\left(\hat{\theta}^{\#}\right) \chi\left(\hat{\theta}^{\#}\right)\right)
$$

where $\chi\left(\hat{\theta}^{\#}\right)$ is the matrix of sensitivities with entries given in (3.8). The standard error estimates are again given by the square roots of the diagonal entries of the covariance matrix

$$
\operatorname{SE}\left(\hat{\theta}_{k}^{\#}\right)=\sqrt{\Sigma_{k k}\left(\hat{\theta}^{\#}\right)} .
$$




\subsection{Simulated Generalized Least Squares Data}

As in the previous section, we investigate the well-posedness of the GLS parameter estimation procedure by considering the resulting parameter estimates and uncertainty estimates for simulated data. We simulate data motivated by the GLS error assumptions in (4.1) and (4.2) using both the solutions $u^{\text {rand }}$ of (2.1) and solutions $U$ of (2.2). In order to consider $U$ the solution of (2.2) as a model solution in the inverse problem, we will simulate data which is given by realizations of $\mathbf{D}_{i j}$ where $\mathbf{D}_{i j}$ is given by

$$
\mathbf{D}_{i j}(\sigma)=U_{i j}\left(10^{-3}, 2.9167,0.01\right)\left(1+\sigma \boldsymbol{B}_{i j}\right)
$$

where $U_{i j}\left(10^{-3}, 2.9167,0.01\right)$ is given by $(3.1)$, and $\boldsymbol{B}_{i j}$ follows a standard normal distribution or $\boldsymbol{B}_{i j} \sim \mathcal{N}\left(0,1^{2}\right)$. Again, as in Section 3.1, we consider spatial nodes $x_{i}=0,0.57,1.14$ and $x_{i}=0,0.57$ and temporal nodes $t_{j}=0.6+\frac{1}{120}, 0.6+\frac{2}{120}, \ldots, 0.6+\frac{7}{120}, 20,40, \ldots, 140$. We analyzed five realizations of $\mathbf{D}_{i j}(\sigma)$ for each value of $\sigma=0.02,0.05,0.10$.

We also consider data which is generated using solutions of (2.1) and motivated by the GLS assumptions with realizations of the random process

$$
\mathbf{D}_{i j}^{\text {rand }}(\sigma)=u_{i j}^{\text {rand }}\left(1+\sigma \boldsymbol{B}_{i j}\right),
$$

where $u_{i j}^{\text {rand }}$ is defined in (3.16), and $\boldsymbol{B}_{i j}$ is a random variable sampled from a standard normal distribution or $\boldsymbol{B}_{i j} \sim \mathcal{N}\left(0,1^{2}\right)$. We calculated five realizations of $\mathbf{D}_{i j}^{\text {rand }}(\sigma)$ for each value of $\sigma=0,0.02,0.05,0.10$.

For both sets of simulations, we calculate the parameter estimates $\hat{\theta}^{\#}$, or $\hat{\theta}^{\lambda}=\left(\hat{\gamma}_{\lambda}, \hat{\alpha}_{\lambda}\right)$, $\hat{\theta}^{\gamma}=\left(\hat{\alpha}_{\gamma}, \hat{\lambda}_{\gamma}\right)$ and $\hat{\theta}=(\hat{\gamma}, \hat{\alpha}, \hat{\lambda})$ as defined in (4.4) by minimizing (4.3). We also calculate $\operatorname{SE}\left(\hat{\theta}^{\#}\right)$ using (4.8).

As in Section 3.1, we consider the accuracy of the inverse problem by investigating $\hat{\theta}^{\#}-\theta_{0}^{\#}$ and the uncertainty associated with the inverse problem by investigating the ratios $\operatorname{SE}\left(\hat{\theta}^{\#}\right) / \hat{\theta}^{\#}$. In Figures $4.1-4.6$, we report on these simulations with spatial nodes $x_{i}=0,0.57,1.14$. Figure 4.1 (a) depicts $\hat{\alpha}_{\#}-\alpha_{0}$ for five realizations of $\mathbf{D}_{i j}(\sigma)$ for each value of $\sigma=0.02,0.05,0.10$. The values of $\hat{\alpha}_{\#}-\alpha_{0}$ in Figure $4.1(\mathrm{~b})$ for five realizations of $\mathbf{D}_{i j}^{\text {rand }}(\sigma)$ for each value of $\sigma=0,0.02,0.05,0.10$ appear to be smaller than those in Figure 4.1(a). This suggests that the GLS parameter estimation procedure predicts $\hat{\alpha}_{\#}$ more accurately for data generated by realizations $\mathbf{D}_{i j}^{\text {rand }}(\sigma)$ than for data generated by realizations of $\mathbf{D}_{i j}(\sigma)$.

To consider the uncertainty associated with the parameter estimates $\hat{\alpha}_{\#}-\alpha_{0}$, we plotted the ratio $\operatorname{SE}\left(\hat{\alpha}_{\#}\right) / \hat{\alpha}_{\#}$ in Figure $4.2(\mathrm{a})$ for realizations of $\mathbf{D}_{i j}(\sigma)$ and in Figure $4.2(\mathrm{~b})$ for realizations of $\mathbf{D}_{i j}^{\text {rand }}(\sigma)$. The ratios $\operatorname{SE}\left(\hat{\alpha}_{\#}\right) / \hat{\alpha}_{\#}$ appear to be linear in $\sigma$ for realizations of $\mathbf{D}_{i j}(\sigma)$ and $\mathbf{D}_{i j}^{\text {rand }}(\sigma)$ with similar slopes. It appears that there is little difference between using realizations of $\mathbf{D}_{i j}(\sigma)$ and realizations of $\mathbf{D}_{i j}^{\text {rand }}(\sigma)$ in the GLS estimate of the uncertainty associated with the parameter estimate $\hat{\alpha}_{\#}$.

We plotted the differences $\hat{\lambda}_{\#}-\lambda_{0}$ for the realizations of $\mathbf{D}_{i j}(\sigma)$ in Figure $4.3(\mathrm{a})$ and the realizations of $\mathbf{D}_{i j}^{\text {rand }}(\sigma)$ in Figure $4.3(\mathrm{~b})$. The differences between $\hat{\lambda}_{\gamma}$ and $\lambda_{0}$ are very small for both realizations of $\mathbf{D}_{i j}(\sigma)$ in Figure 4.3(a) and realizations of $\mathbf{D}_{i j}^{\text {rand }}(\sigma)$ in Figure 4.3(b). The 


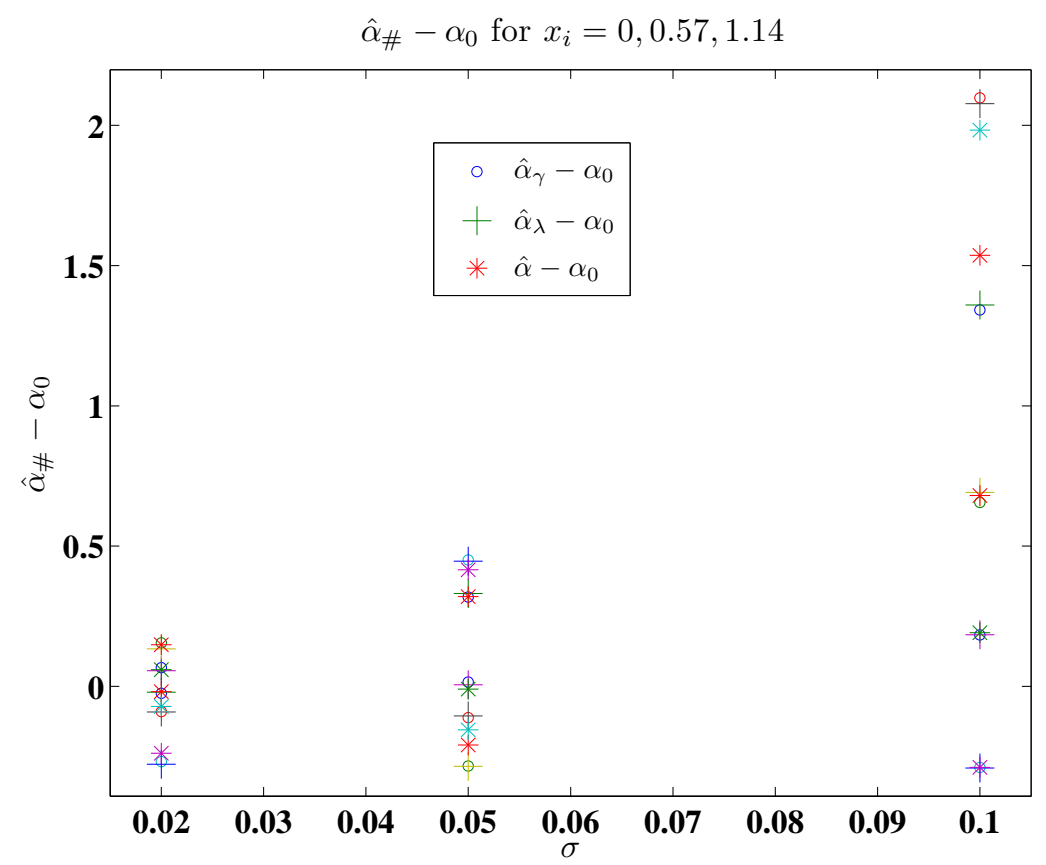

(a)

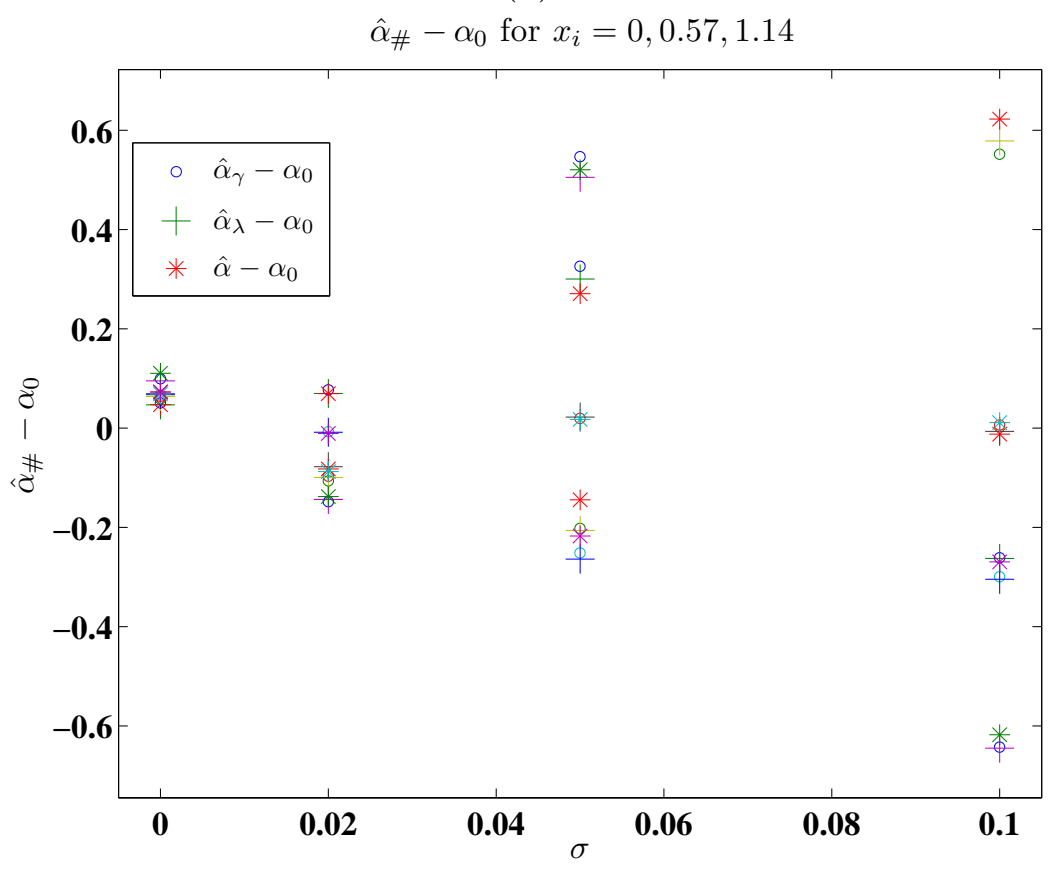

(b)

Figure 4.1: The results of realizations of simulated data with temporal nodes $t_{j}=0.6+$ $\frac{1}{120}, 0.6+\frac{2}{120}, \ldots, \frac{7}{120}, 20,40, \ldots, 140$ and spatial nodes $x_{i}=0,0.57,1.14$. The points denoted with ${ }^{o}$ are the difference $\hat{\alpha}_{\gamma}-\alpha_{0}$, the points denoted are the $\hat{\alpha}_{\lambda}-\alpha_{0}$, the points denoted * are the difference $\hat{\alpha}-\alpha_{0}$ (a) The result of five realizations of $\mathbf{D}_{i j}(\sigma)$ for values of $\sigma=$ $0.02,0.05,0.10$ (b) The result of five realizations of $\mathbf{D}_{i j}^{\text {rand }}(\sigma)$ for values of $\sigma=0,0.02,0.10$. 
$\operatorname{SE}\left(\hat{\alpha}_{\#}\right) / \hat{\alpha}_{\#}$ for $x_{i}=0,0.57,1.14$

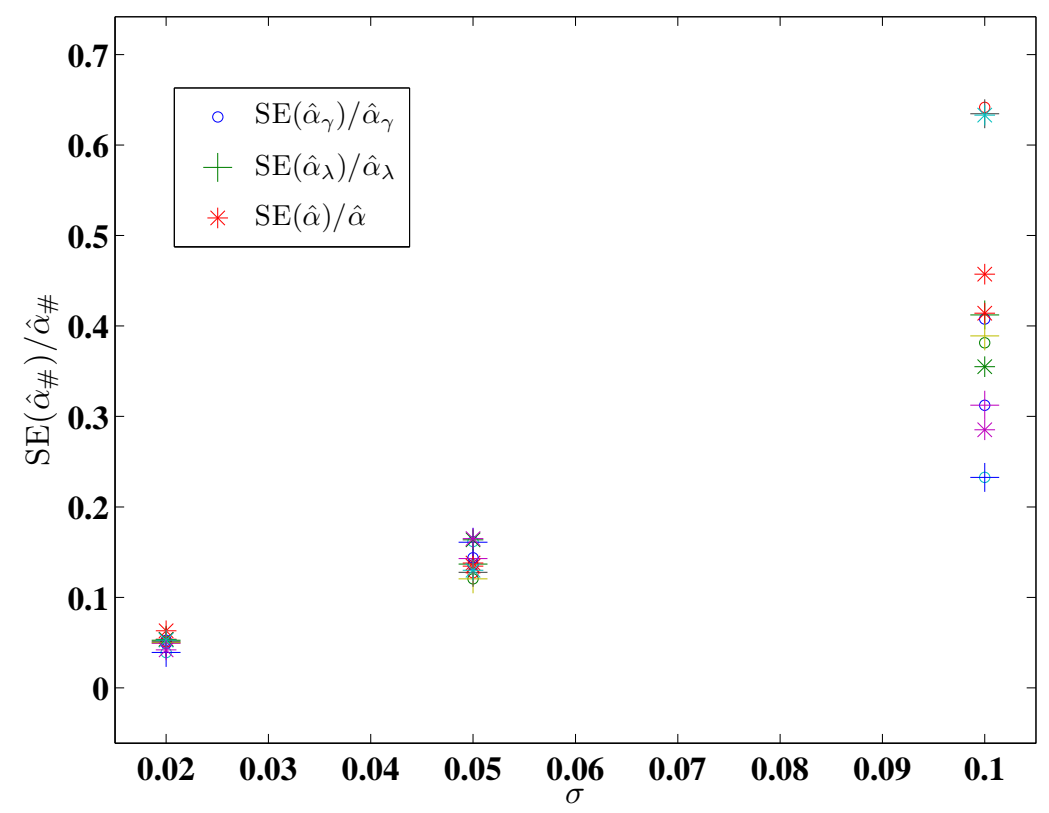

(a)

$\operatorname{SE}\left(\hat{\alpha}_{\#}\right) / \hat{\alpha}_{\#}$ for $x_{i}=0,0.57,1.14$

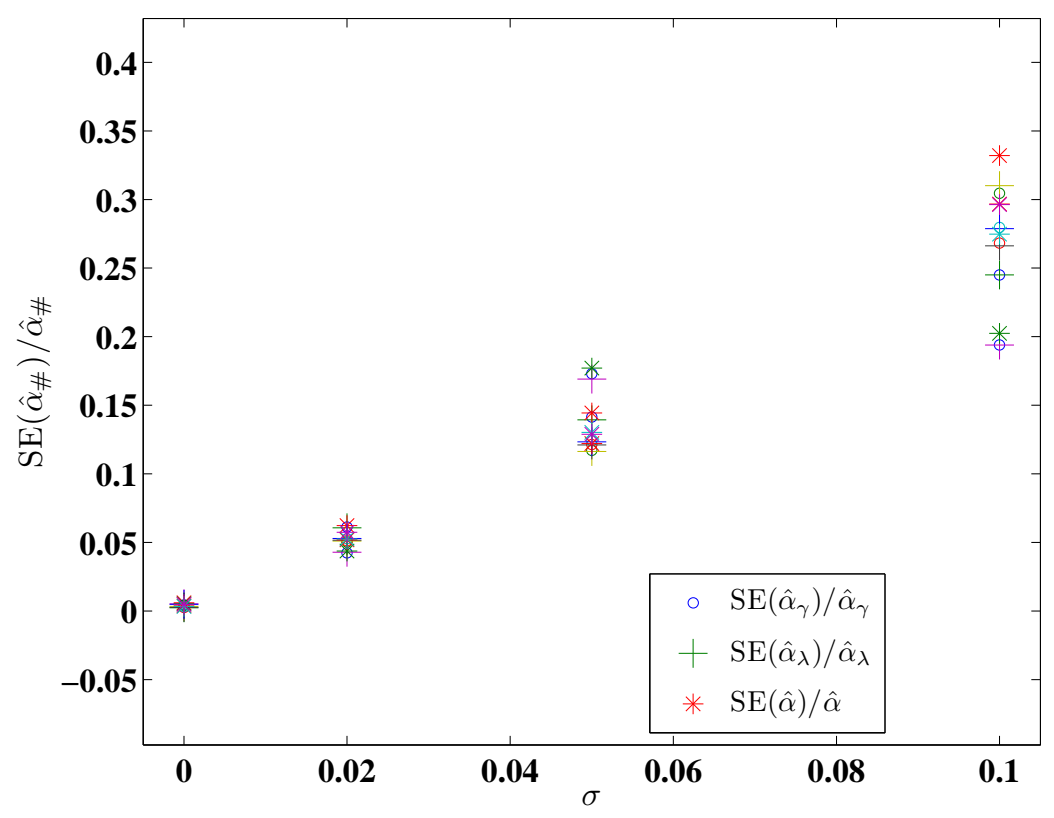

(b)

Figure 4.2: The results of realizations of simulated data with temporal nodes $t_{j}=$ $0.6+\frac{1}{120}, 0.6+\frac{2}{120}, \ldots, \frac{7}{120}, 20,40, \ldots, 140$ and spatial nodes $x_{i}=0,0.57,1.14$. The points denoted with ${ }^{o}$ are the ratio $\operatorname{SE}\left(\hat{\alpha}_{\gamma}\right) / \hat{\alpha}_{\gamma}$, the points denoted are the $\operatorname{SE}\left(\hat{\alpha}_{\lambda}\right) / \hat{\alpha}_{\lambda}$, the points denoted $^{*}$ are the difference $\operatorname{SE}(\hat{\alpha}) / \hat{\alpha}$ (a) The result of five realizations of $\mathbf{D}_{i j}(\sigma)$ for values of $\sigma=0.02,0.05,0.10$ (b) The result of five realizations of $\mathbf{D}_{i j}^{\text {rand }}(\sigma)$ for values of $\sigma=0,0.02,0.05,0.10$. 
differences $\hat{\lambda}-\lambda_{0}$ (depicted in Figure 4.3(a) for realizations of $\mathbf{D}_{i j}(\sigma)$ and Figure 4.3(b) for realizations of $\left.\mathbf{D}_{i j}^{\text {rand }}(\sigma)\right)$ have much larger magnitudes than the magnitudes of the differences $\hat{\lambda}_{\gamma}-\lambda_{0}$. This suggests, as discussed in Section 3.1 (see the discussions involving (3.11)(3.13)), that estimating $\gamma$ detracts from the accuracy of the GLS estimate of the parameter $\lambda$.

We further see the effect of estimating $\gamma$ on the uncertainty associated with the parameter estimate $\hat{\lambda}$. In Figure $4.4($ a $)$ we plotted the ratio $\operatorname{SE}\left(\hat{\lambda}_{\gamma}\right) / \hat{\lambda}_{\gamma}$ versus $\sigma$ for realizations of $\mathbf{D}_{i j}(\sigma)$ and the ratio $\operatorname{SE}\left(\hat{\lambda}_{\gamma}\right) / \hat{\lambda}_{\gamma}$ versus $\sigma$ in Figure $4.4(\mathrm{c})$. The ratios $\operatorname{SE}\left(\hat{\lambda}_{\gamma}\right) / \hat{\lambda}_{\gamma}$ in both of these examples appear to be linearly dependent on $\sigma$. When the full parameter set $\hat{\theta}$ is estimated, we see that the ratio $\operatorname{SE}\left(\hat{\lambda}_{\gamma}\right) / \hat{\lambda}_{\gamma}$ varies exponentially with $\sigma$ in Figures (b) and (d) in which we plotted $\log (\mathrm{SE}(\hat{\alpha}) / \hat{\alpha})$ versus $\sigma$ for realizations of $\mathbf{D}_{i j}(\sigma)$ and $\mathbf{D}_{i j}^{\text {rand }}(\sigma)$, respectively. 


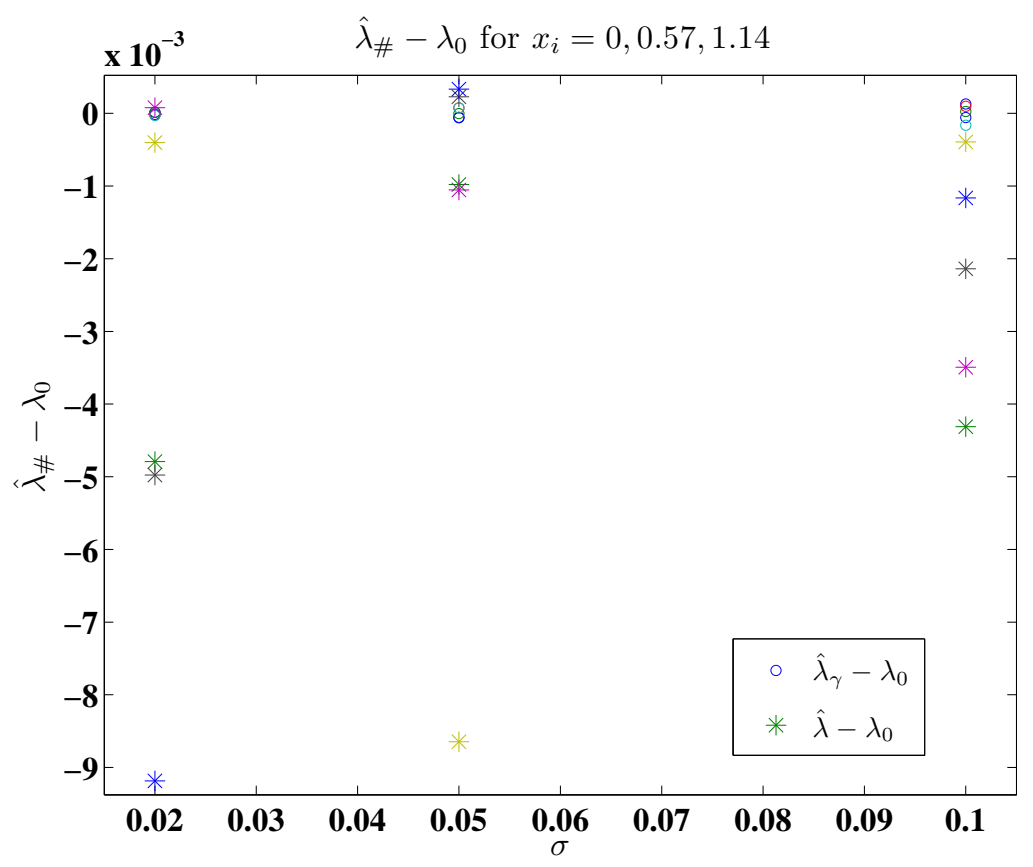

(a)

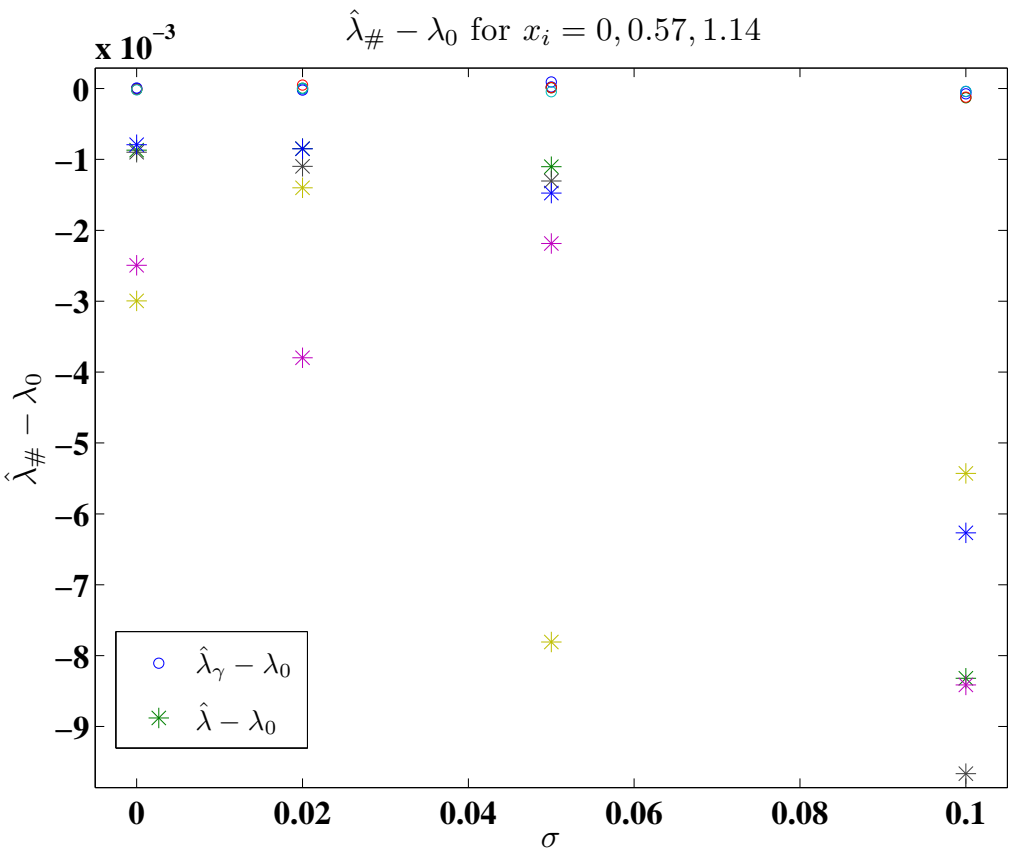

(b)

Figure 4.3: The results of realizations of simulated data with temporal nodes $t_{j}=0.6+$ $\frac{1}{120}, 0.6+\frac{2}{120}, \ldots, \frac{7}{120}, 20,40, \ldots, 140$ and spatial nodes $x_{i}=0,0.57,1.14$. The points denoted with ${ }^{o}$ are the difference $\hat{\lambda}_{\gamma}-\lambda_{0}$, and the points denoted * are the difference $\hat{\lambda}-\lambda_{0}$ (a) The result of five realizations of $\mathbf{D}_{i j}(\sigma)$ for values of $\sigma=0.02,0.05,0.10$ (b) The result of five realizations of $\mathbf{D}_{i j}^{\text {rand }}(\sigma)$ for values of $\sigma=0,0.02,0.05,0.10$. 


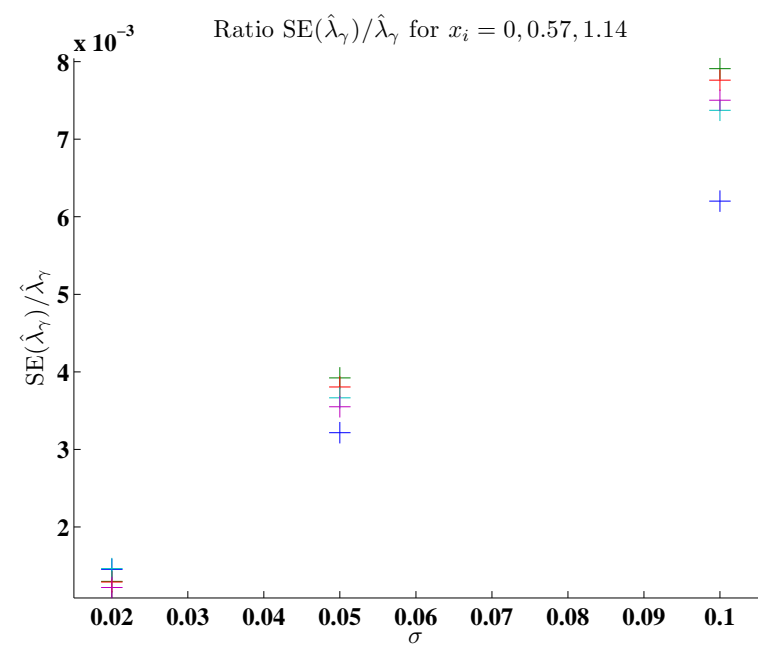

(a)

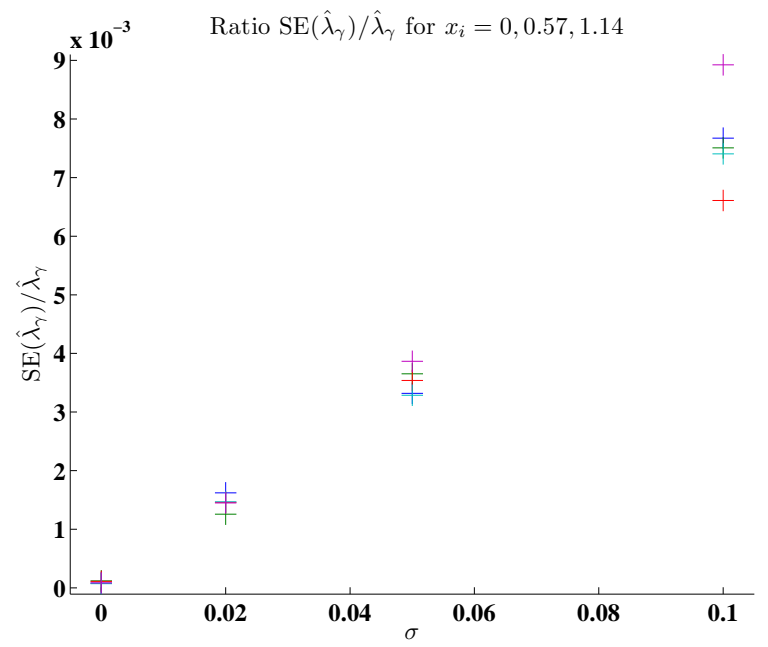

(c)

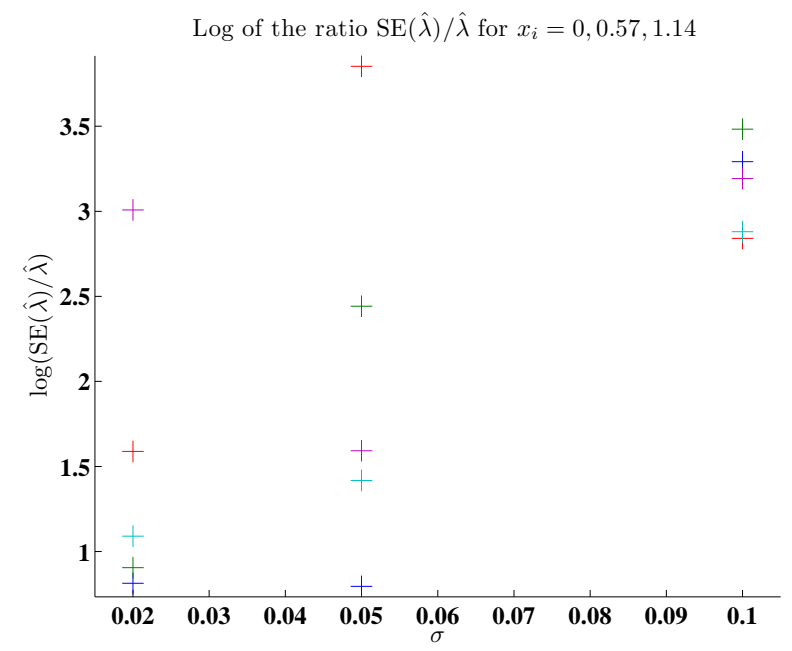

(b)

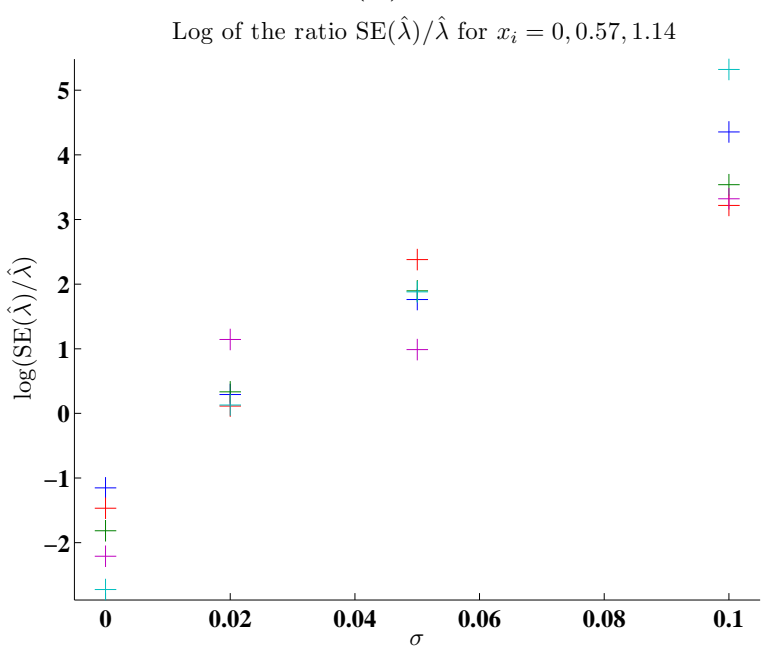

(d)

Figure 4.4: The results of realizations of simulated data with temporal nodes $t_{j}=$ $0.6+\frac{1}{120}, 0.6+\frac{2}{120}, \ldots, \frac{7}{120}, 20,40, \ldots, 140$ and spatial nodes $x_{i}=0,0.57,1.14$. (a) The ratio $\operatorname{SE}\left(\hat{\lambda}_{\gamma}\right) / \hat{\lambda}_{\gamma}$ for five realizations of $\mathbf{D}_{i j}(\sigma)$ for values of $\sigma=0.02,0.05,0.10$ (b) The log of the ratio $\log \mathrm{SE}(\hat{\lambda}) / \hat{\lambda}$ for five realizations of $\mathbf{D}_{i j}(\sigma)$ for values of $\sigma=0.02,0.05,0.10$. (c) The ratio $\operatorname{SE}\left(\hat{\lambda}_{\gamma}\right) / \hat{\lambda}_{\gamma}$ for five realizations of $\mathbf{D}_{i j}^{\text {rand }}(\sigma)$ for values of $\sigma=0,0.02,0.05,0.10$. (d) The log of the ratio $\log \operatorname{SE}(\hat{\lambda}) / \hat{\lambda}$ for five realizations of $\mathbf{D}_{i j}^{\text {rand }}(\sigma)$ for values of $\sigma=0,0.02,0.05,0.10$. 
We also examined the differences between $\hat{\gamma}_{\#}-\gamma_{0}$ in Figures $4.5(\mathrm{a})$ and (b). In Figure $4.5(\mathrm{a})$, we plotted $\hat{\gamma}_{\#}-\gamma_{0}$ for realizations of $\mathbf{D}_{i j}(\sigma)$. In Figure $4.5(\mathrm{~b})$, we plotted $\hat{\gamma}_{\#}-\gamma_{0}$ for realizations of $\mathbf{D}_{i j}^{\text {rand }}(\sigma)$. In both Figure 4.5(a) and (b), $\left|\hat{\gamma}_{\lambda}-\gamma_{0}\right|$ is several orders of magnitude less than $\left|\hat{\gamma}-\gamma_{0}\right|$. Thus by estimating the entire parameter set $\hat{\theta}$, we gain inaccuracy of our estimate of $\hat{\gamma}$. Moreover, the differences $\left|\hat{\gamma}-\gamma_{0}\right|$ are an order of magnitude greater than the parameter itself $\gamma_{0}=10^{-3}$. In Figures $4.6(\mathrm{a})-(\mathrm{b})$, we see that the uncertainty associated with the parameter estimate $\hat{\gamma}_{\#}$ varies exponentially with $\sigma$. Figure 4.6(a) and (c), we plotted $\log \left(\operatorname{SE}\left(\hat{\gamma}_{\lambda}\right) / \hat{\gamma}_{\lambda}\right)$ for realizations of $\mathbf{D}_{i j}(\sigma)$ and $\mathbf{D}_{i j}^{\text {rand }}(\sigma)$, respectively. In Figures $4.6(\mathrm{~b})$ and $(\mathrm{d})$, we see that $\log (\mathrm{SE}(\hat{\gamma}) / \hat{\gamma})$ is larger for realizations of $\mathbf{D}_{i j}(\sigma)$ (in (b)) than for realizations of $\mathbf{D}_{i j}^{\text {rand }}(\sigma)$ (in $\left.(\mathrm{d})\right)$ though $\log (\mathrm{SE}(\hat{\gamma}) / \hat{\gamma})$ (in Figures $4.6(\mathrm{~b})$ and $(\mathrm{d})$ ) appears to be larger than $\log \left(\operatorname{SE}\left(\hat{\gamma}_{\lambda}\right) / \hat{\gamma}_{\lambda}\right.$ ) (in Figure $4.6(\mathrm{a})$ and $(\mathrm{c})$ ). 


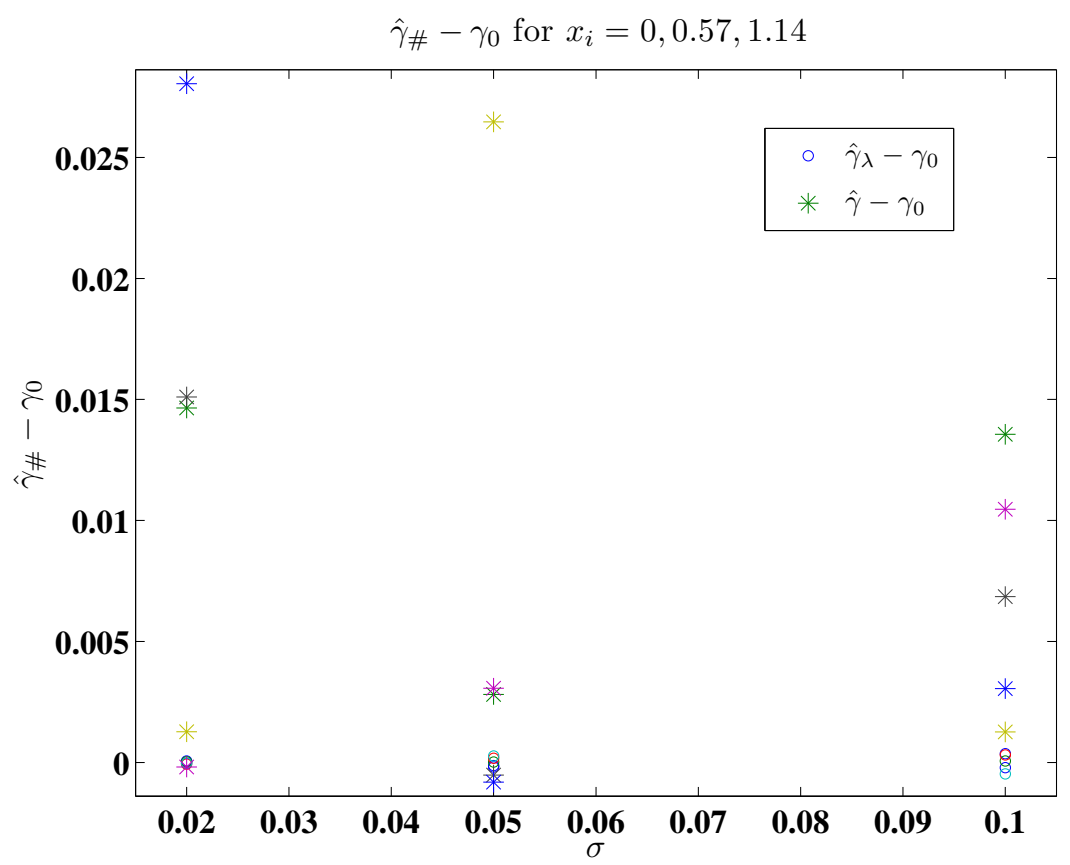

(a)

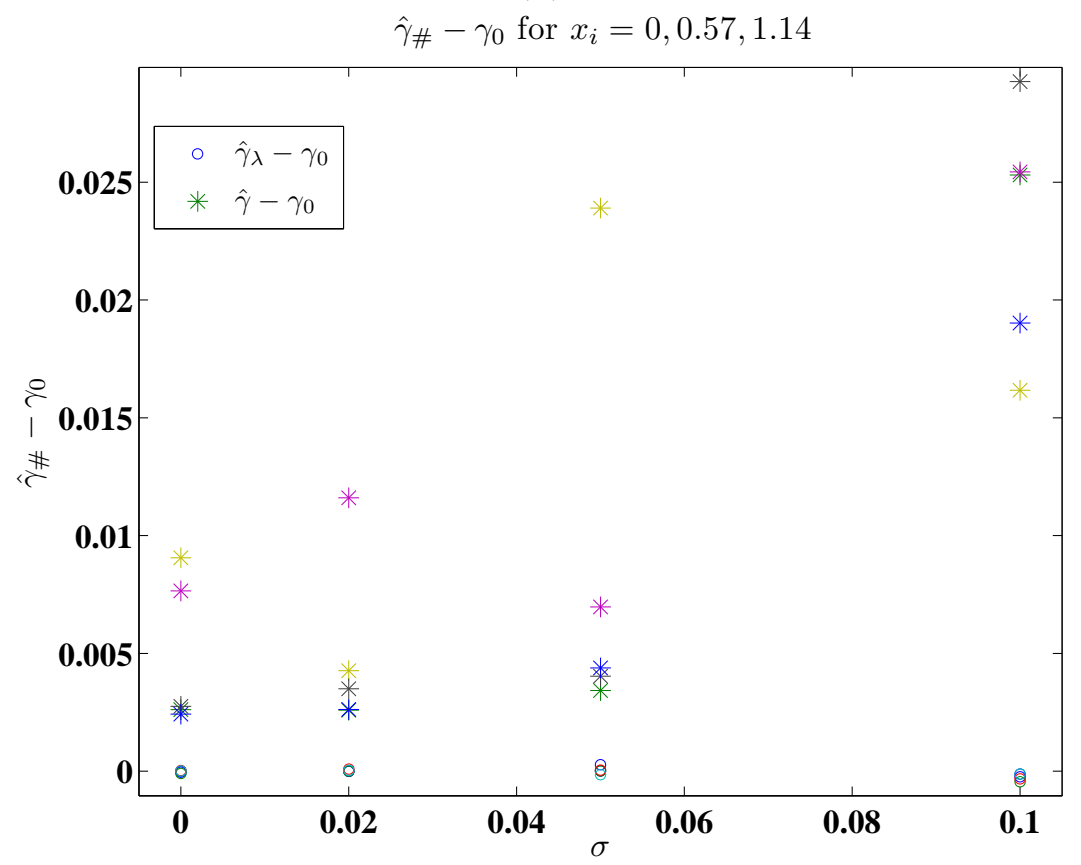

(b)

Figure 4.5: The results of realizations of simulated data with temporal nodes $t_{j}=0.6+$ $\frac{1}{120}, 0.6+\frac{2}{120}, \ldots, \frac{7}{120}, 20,40, \ldots, 140$ and spatial nodes $x_{i}=0,0.57,1.14$. The points denoted with ${ }^{o}$ are the difference $\hat{\gamma}_{\lambda}-\gamma_{0}$, and the points denoted ${ }^{*}$ are the difference $\hat{\gamma}-\gamma_{0}$ (a) The result of five realizations of $\mathbf{D}_{i j}(\sigma)$ for values of $\sigma=0.02,0.05,0.10$ (b) The result of five realizations of $\mathbf{D}_{i j}^{\text {rand }}(\sigma)$ for values of $\sigma=0,0.02,0.05,0.10$. 


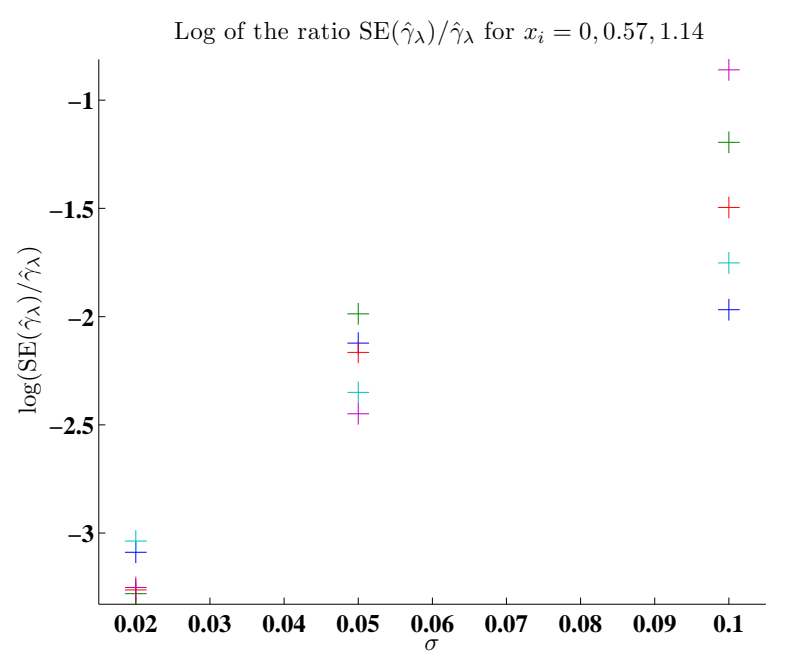

(a)

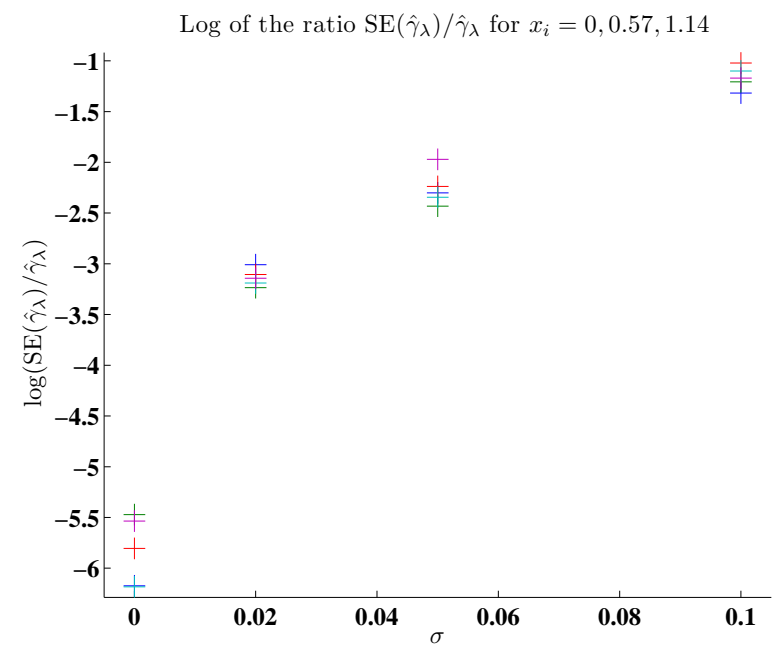

(c)

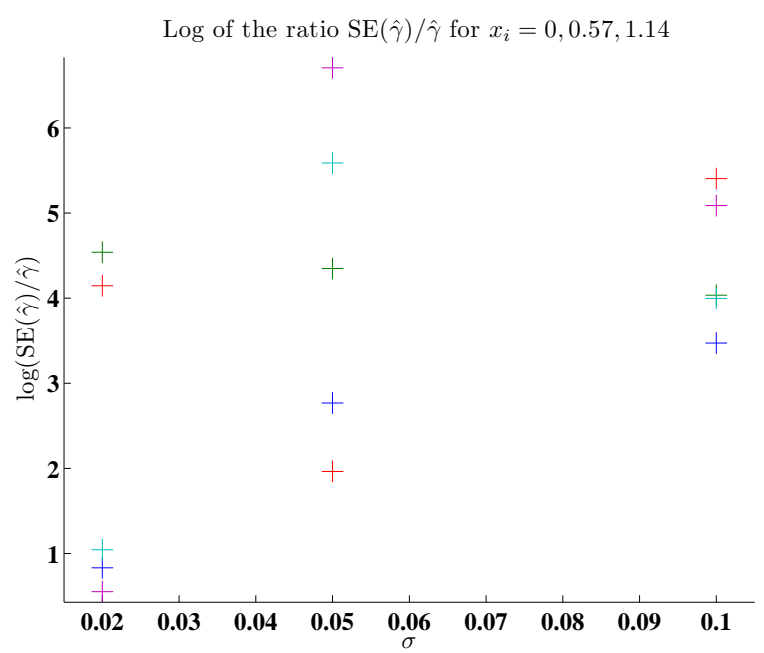

(b)

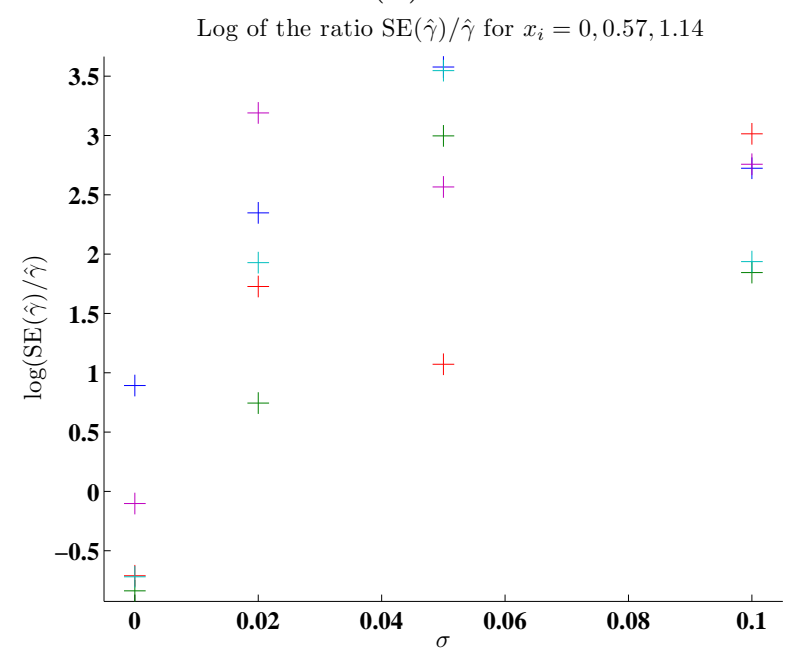

(d)

Figure 4.6: The results of realizations of simulated data with temporal nodes $t_{j}=0.6+$ $\frac{1}{120}, 0.6+\frac{2}{120}, \ldots, \frac{7}{120}, 20,40, \ldots, 140$ and spatial nodes $x_{i}=0,0.57,1.14$. (a) The log of the ratio $\log \left(\mathrm{SE}\left(\hat{\gamma}_{\lambda}\right) / \hat{\gamma}_{\lambda}\right)$ for five realizations of $\mathbf{D}_{i j}(\sigma)$ for values of $\sigma=0.02,0.05,0.10$ (b) The $\log$ of the ratio $\log (\mathrm{SE}(\hat{\gamma}) / \hat{\gamma})$ for five realizations of $\mathbf{D}_{i j}(\sigma)$ for values of $\sigma=0.02,0.05,0.10$. (c) The $\log$ of the ratio $\log \left(\operatorname{SE}\left(\hat{\gamma}_{\lambda}\right) / \hat{\gamma}_{\lambda}\right)$ for five realizations of $\mathbf{D}_{i j}^{\text {rand }}(\sigma)$ for values of $\sigma=$ $0,0.02,0.05,0.10$. (d) The $\log$ of the ratio $\log \mathrm{SE}(\hat{\gamma}) / \hat{\gamma}$ for five realizations of $\mathbf{D}_{i j}^{\text {rand }}(\sigma)$ for values of $\sigma=0,0.02,0.05,0.10$. 
We also considered realizations of $\mathbf{D}_{i j}(\sigma)$ (given by (4.9)) and $\mathbf{D}_{i j}^{\text {rand }}(\sigma)$ (given by (4.10)) for spatial nodes $x_{i}=0,0.57$ and the same temporal nodes $t_{j}=0.6+\frac{1}{120}, 0.6+\frac{2}{120}, \ldots, \frac{7}{120}, 20$, $40, \ldots, 140$. The results of these realizations are depicted in Figures 4.7-4.12.

We see that for realizations of $\mathbf{D}_{i j}(\sigma)$ and $\mathbf{D}_{i j}^{\text {rand }}(\sigma)$ the accuracy of the GLS parameter estimation procedure for the parameter $\alpha$ is similar for the two random processes. We see this in Figures 4.7(a) and (b) though it does appear that one realization of $\mathbf{D}_{i j}^{\text {rand }}(0.10)$ produced large values of $\hat{\alpha}_{\lambda}, \hat{\alpha}_{\gamma}$, and $\hat{\alpha}$ in Figure 4.7(b). In Figures 4.8(b), we see this extreme realization of $\mathbf{D}_{i j}^{\text {rand }}(0.10)$ also produced large ratios $\operatorname{SE}\left(\hat{\alpha}_{\#}\right) / \hat{\alpha}_{\#}$. Other than this extreme realization, the ratios $\operatorname{SE}\left(\hat{\alpha}_{\#}\right) / \hat{\alpha}_{\#}$ for realizations of $\mathbf{D}_{i j}(\sigma)$ (in Figure 4.8(a)) and $\mathbf{D}_{i j}^{\text {rand }}(\sigma)$ (in Figure 4.8(b)) appear to have similar linear dependence on $\sigma$.

The differences $\hat{\lambda}_{\#}-\lambda_{0}$ are depicted in Figures 4.9(a) and (b) for realizations of $\mathbf{D}_{i j}(\sigma)$ and $\mathbf{D}_{i j}^{\text {rand }}(\sigma)$, respectively. Again, we see similar results for realizations of $\mathbf{D}_{i j}(\sigma)$ and realizations of $\mathbf{D}_{i j}^{\text {rand }}(\sigma)$. We also note, that as was the case for spatial nodes $x_{i}=0,0.57,1.14$ in Figure 4.3(a) and (b), $\left|\hat{\lambda}-\lambda_{0}\right|$ is much larger than $\left|\hat{\lambda}_{\gamma}-\lambda_{0}\right|$ for both realizations of $\mathbf{D}_{i j}(\sigma)$ and realizations of $\mathbf{D}_{i j}^{\text {rand }}(\sigma)$. The ratio $\operatorname{SE}\left(\hat{\lambda}_{\gamma}\right) / \hat{\lambda}_{\gamma}$ appears to vary linearly with $\sigma$ for both realizations of $\mathbf{D}_{i j}(\sigma)$ (in Figure 4.10(a)) and realizations of $\mathbf{D}_{i j}^{\text {rand }}(\sigma)$ (in Figure 4.10(c)), while the dependence of $\operatorname{SE}(\hat{\lambda}) / \hat{\lambda}$ is less clear though it does vary greatly with $\sigma$ for both realizations $\mathbf{D}_{i j}(\sigma)(\log (\operatorname{SE}(\hat{\lambda}) / \hat{\lambda})$ is plotted in Figure $4.10(\mathrm{~b}))$ and realizations of $\mathbf{D}_{i j}^{\text {rand }}(\sigma)$ $(\log (\operatorname{SE}(\hat{\lambda}) / \hat{\lambda})$ is plotted in Figure 4.10(d)). 


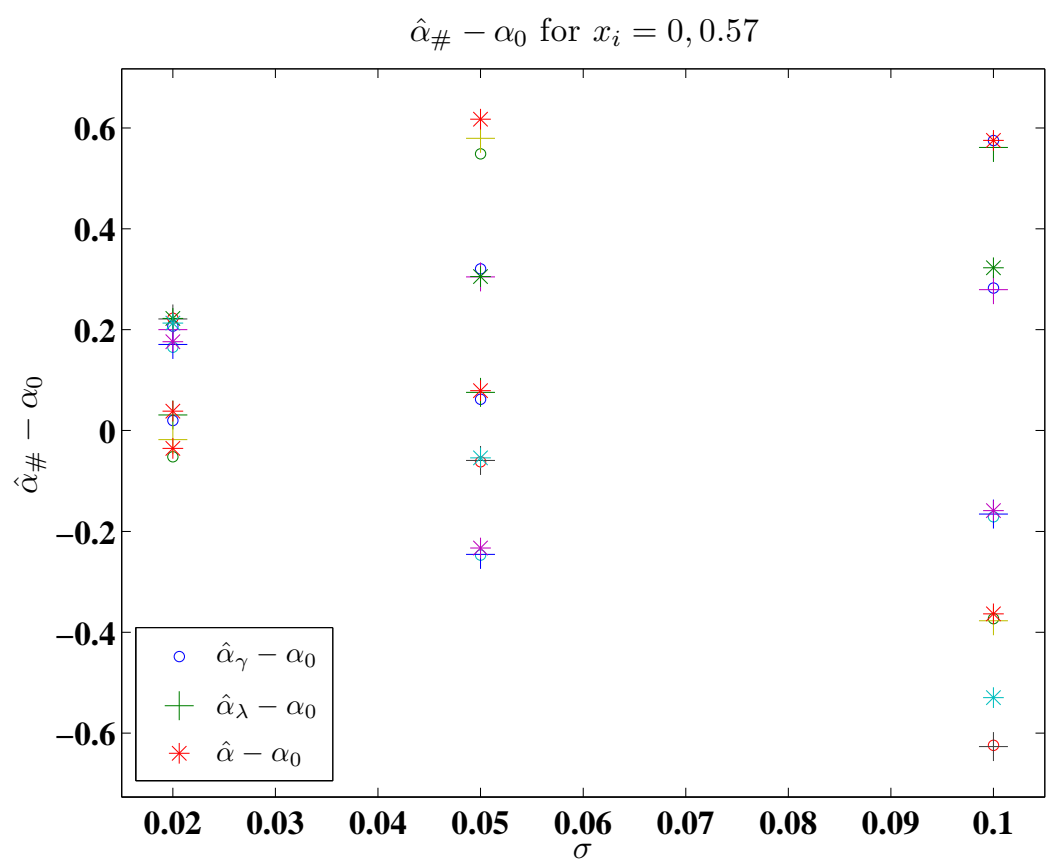

(a)

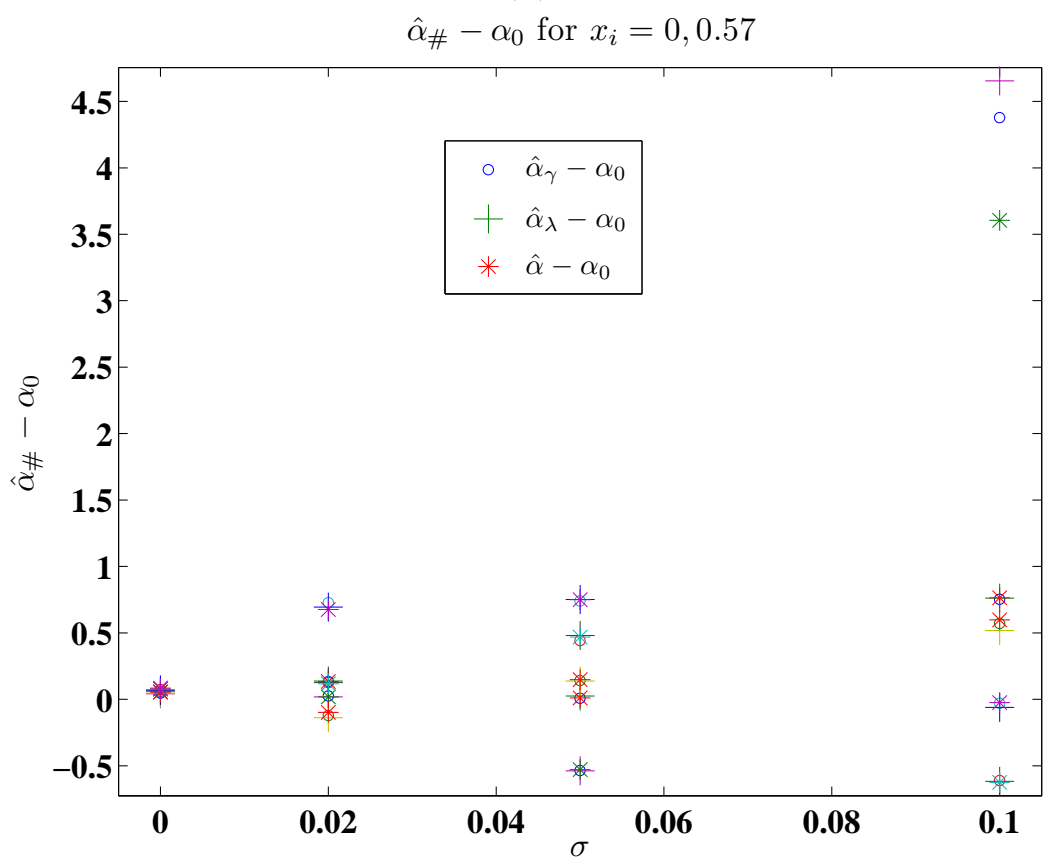

(b)

Figure 4.7: The results of realizations of simulated data with temporal nodes $t_{j}=0.6+$ $\frac{1}{120}, 0.6+\frac{2}{120}, \ldots, \frac{7}{120}, 20,40, \ldots, 140$ and spatial nodes $x_{i}=0,0.57$. The points denoted with ${ }^{o}$ are the difference $\hat{\alpha}_{\gamma}-\alpha_{0}$, the points denoted are the $\hat{\alpha}_{\lambda}-\alpha_{0}$, the points denoted * are the difference $\hat{\alpha}-\alpha_{0}$ (a) The result of five realizations of $\mathbf{D}_{i j}(\sigma)$ for values of $\sigma=0.02,0.05,0.10$ (b) The result of five realizations of $\mathbf{D}_{i j}^{\text {rand }}(\sigma)$ for values of $\sigma=0,0.02,0.05,0.10$. 


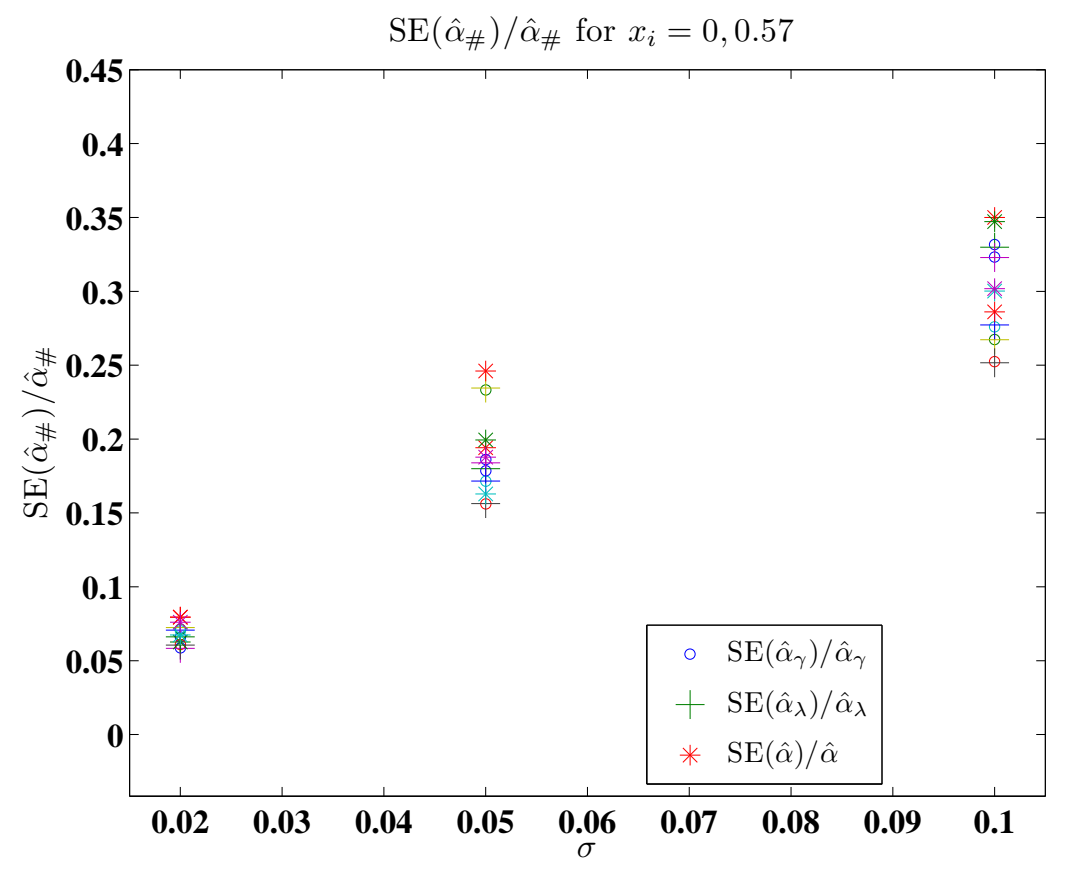

(a)

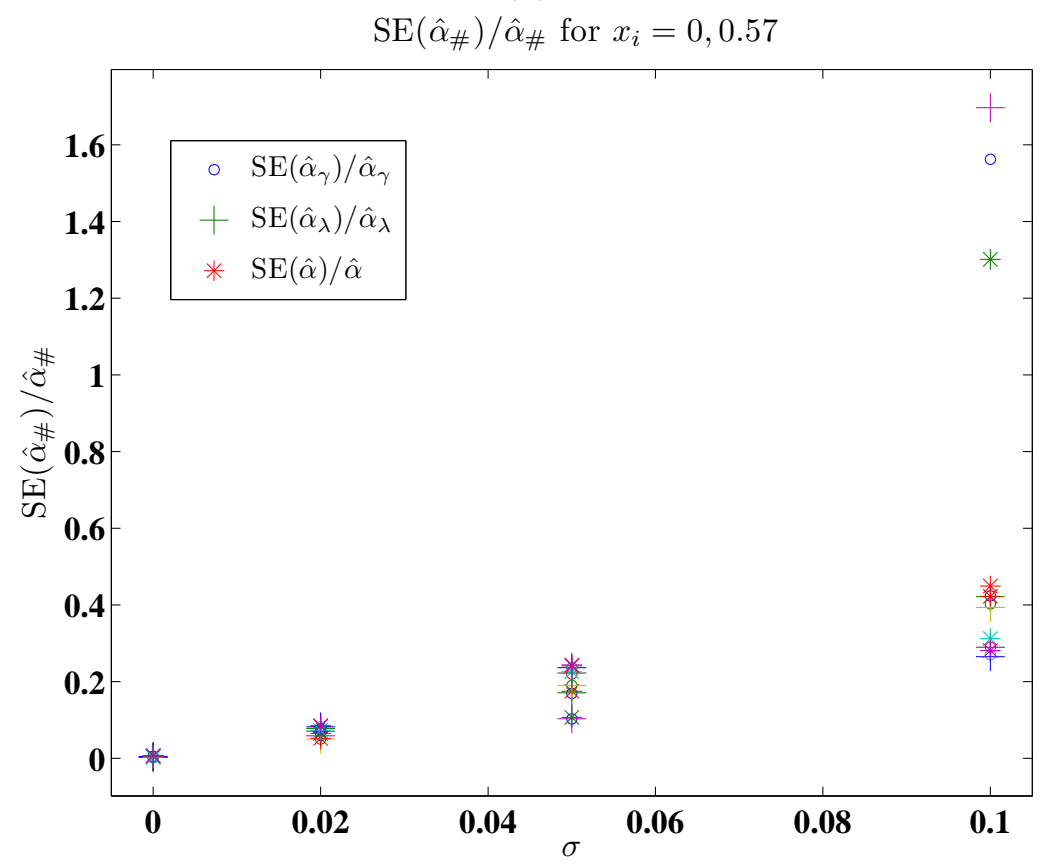

(b)

Figure 4.8: The results of realizations of simulated data with temporal nodes $t_{j}=$ $0.6+\frac{1}{120}, 0.6+\frac{2}{120}, \ldots, \frac{7}{120}, 20,40, \ldots, 140$ and spatial nodes $x_{i}=0,0.57$. The points denoted with ${ }^{o}$ are the ratio $\operatorname{SE}\left(\hat{\alpha}_{\gamma}\right) / \hat{\alpha}_{\gamma}$, the points denoted are the $\operatorname{SE}\left(\hat{\alpha}_{\lambda}\right) / \hat{\alpha}_{\lambda}$, the points denoted * are the difference $\operatorname{SE}(\hat{\alpha}) / \hat{\alpha}$ (a) The result of five realizations of $\mathbf{D}_{i j}(\sigma)$ for values of $\sigma=0.02,0.05,0.10$ (b) The result of five realizations of $\mathbf{D}_{i j}^{\text {rand }}(\sigma)$ for values of $\sigma=0,0.02,0.05,0.10$. 


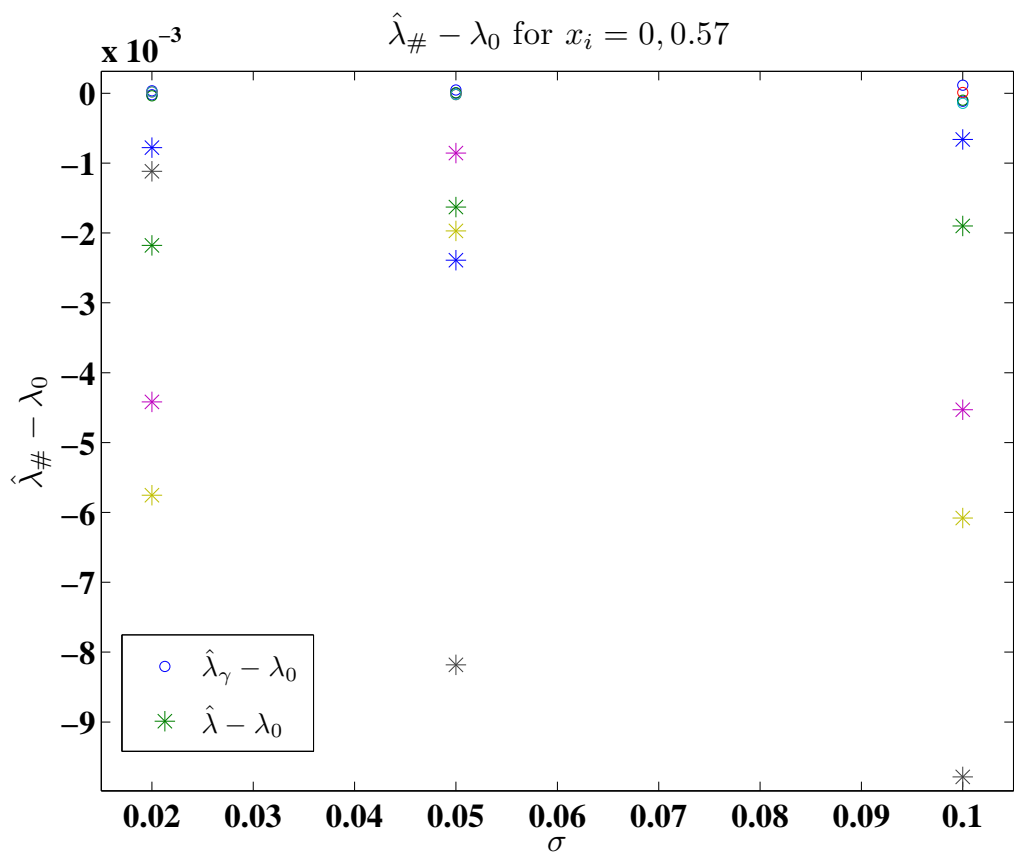

(a)

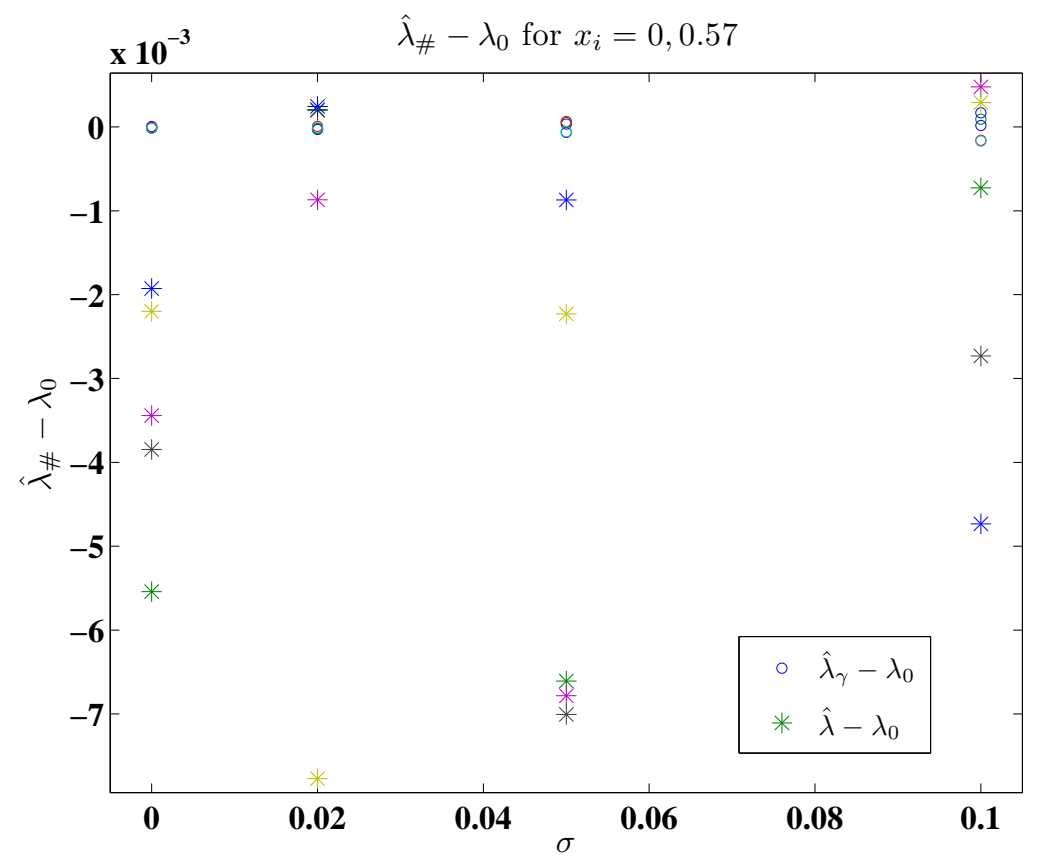

(b)

Figure 4.9: The results of realizations of simulated data with temporal nodes $t_{j}=0.6+$ $\frac{1}{120}, 0.6+\frac{2}{120}, \ldots, \frac{7}{120}, 20,40, \ldots, 140$ and spatial nodes $x_{i}=0,0.57$. The points denoted with ${ }^{o}$ are the difference $\hat{\lambda}_{\gamma}-\lambda_{0}$, and the points denoted ${ }^{*}$ are the difference $\hat{\lambda}-\lambda_{0}$ (a) The result of five realizations of $\mathbf{D}_{i j}(\sigma)$ for values of $\sigma=0.02,0.05,0.10$ (b) The result of five realizations of $\mathbf{D}_{i j}^{\text {rand }}(\sigma)$ for values of $\sigma=0,0.02,0.05,0.10$. 


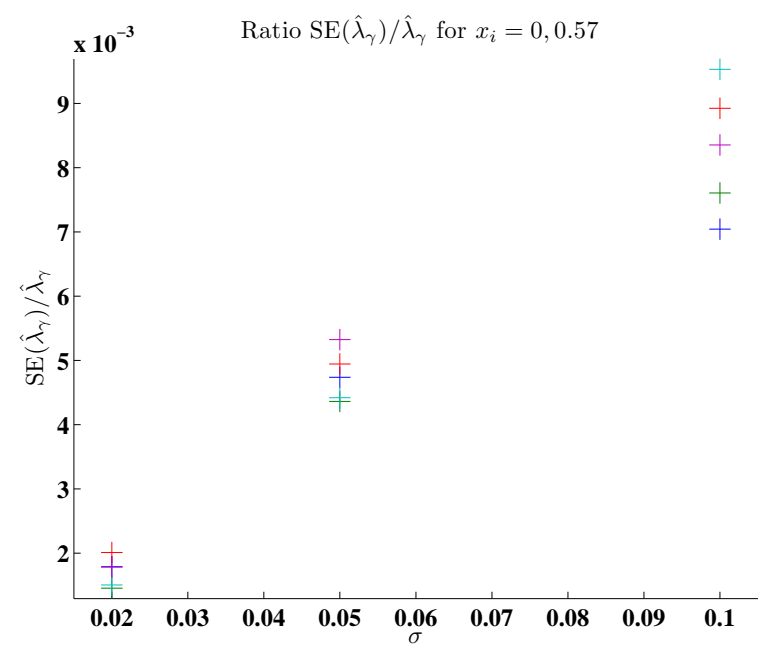

(a)

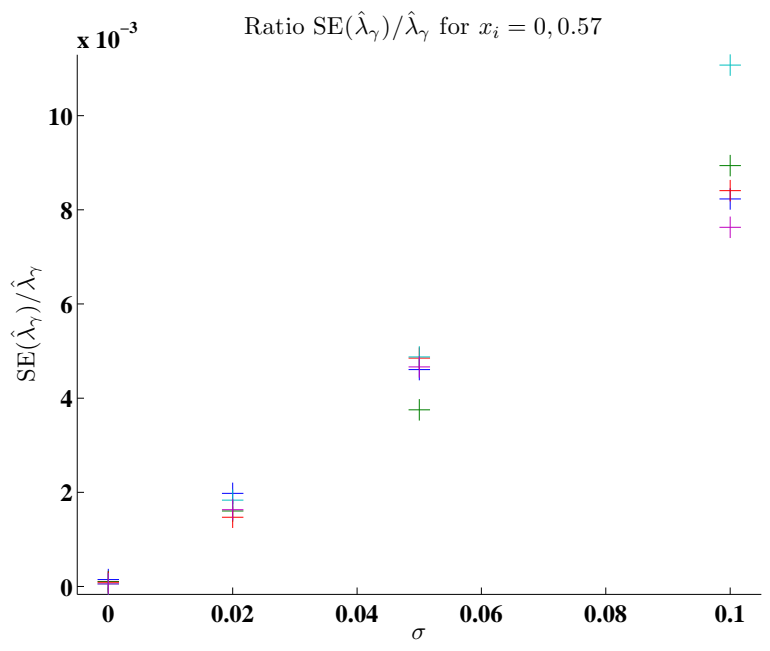

(c)

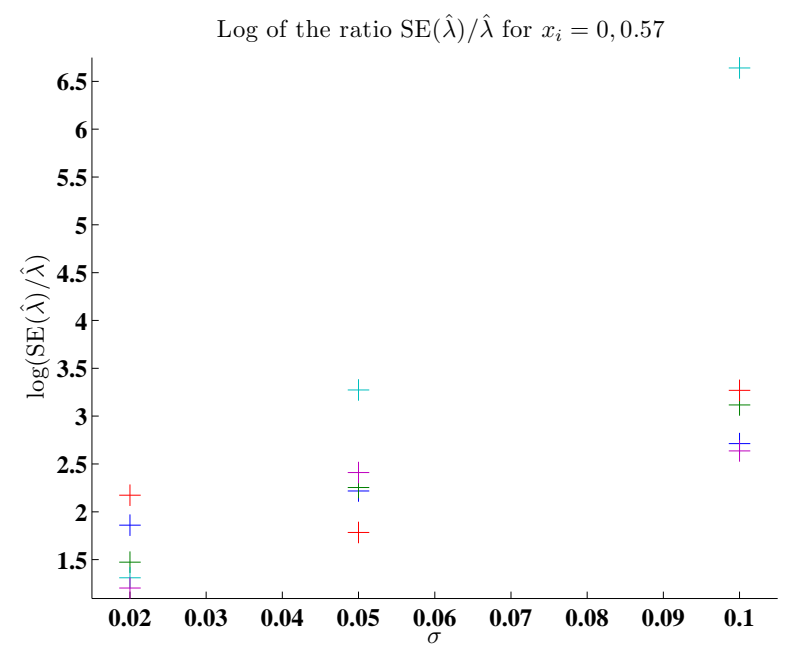

(b)

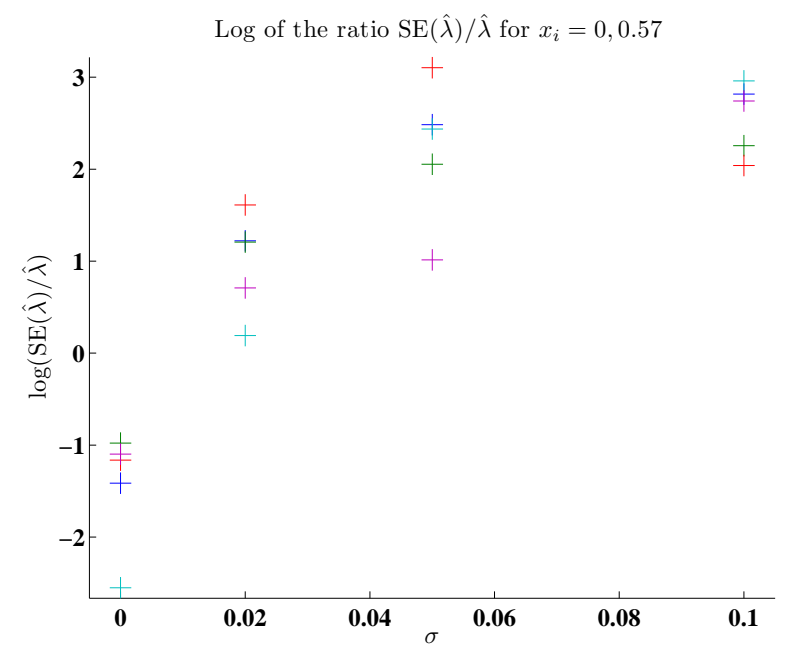

(d)

Figure 4.10: The results of realizations of simulated data with temporal nodes $t_{j}=0.6+$ $\frac{1}{120}, 0.6+\frac{2}{120}, \ldots, \frac{7}{120}, 20,40, \ldots, 140$ and spatial nodes $x_{i}=0,0.57$. (a) The ratio $\operatorname{SE}\left(\hat{\lambda}_{\gamma}\right) / \hat{\lambda}_{\gamma}$ for five realizations of $\mathbf{D}_{i j}(\sigma)$ for values of $\sigma=0.02,0.05,0.10$ (b) The $\log$ of the ratio $\log \operatorname{SE}(\hat{\lambda}) / \hat{\lambda}$ for five realizations of $\mathbf{D}_{i j}(\sigma)$ for values of $\sigma=0.02,0.05,0.10$. (c) The ratio $\operatorname{SE}\left(\hat{\lambda}_{\gamma}\right) / \hat{\lambda}_{\gamma}$ for five realizations of $\mathbf{D}_{i j}^{\text {rand }}(\sigma)$ for values of $\sigma=0,0.02,0.05,0.10$. (d) The log of the ratio $\log \mathrm{SE}(\hat{\lambda}) / \hat{\lambda}$ for five realizations of $\mathbf{D}_{i j}^{\text {rand }}(\sigma)$ for values of $\sigma=0,0.02,0.05,0.10$. 
The differences $\hat{\gamma}_{\#}-\gamma_{0}$ are plotted for realizations of $\mathbf{D}_{i j}(\sigma)$ and $\mathbf{D}_{i j}^{\text {rand }}(\sigma)$ in Figures 4.11(a) and (b), respectively. In both figures, the values $\left|\hat{\gamma}-\gamma_{0}\right|$ are much larger than the values $\left|\hat{\gamma}_{\lambda}-\gamma_{0}\right|$. This demonstrates that in these cases, estimating the full parameter set $\hat{\theta}$ contributes to inaccuracy of the paramter estimate of $\gamma$. Moreover, the differences $\left|\hat{\gamma}-\gamma_{0}\right|$ are several orders of magnitude larger than the "true" parameter value $\gamma_{0}=10^{-3}$. The values of the ratios $\operatorname{SE}\left(\hat{\gamma}_{\#}\right) / \hat{\gamma}_{\#}$ vary a lot with $\sigma$ in Figures $4.12(\mathrm{a})-(\mathrm{d})$. Figure 4.12(a) depicts $\log \left(\operatorname{SE}\left(\hat{\gamma}_{\lambda}\right) / \hat{\gamma}_{\lambda}\right)$ for realizations of $\mathbf{D}_{i j}(\sigma)$ while $\mathbf{D}_{i j}^{\text {rand }}(\sigma)$ depicts $\log \left(\operatorname{SE}\left(\hat{\gamma}_{\lambda}\right) / \hat{\gamma}_{\lambda}\right)$ for realizations of $\mathbf{D}_{i j}^{\text {rand }}(\sigma)$. In both Figure $4.12(\mathrm{a})$ and Figure $4.12(\mathrm{c})$, the ratio $\operatorname{SE}\left(\hat{\gamma}_{\lambda}\right) / \hat{\gamma}_{\lambda}$ appears to depend exponentially on $\sigma$, though the value of $\operatorname{SE}\left(\hat{\gamma}_{\lambda}\right) / \hat{\gamma}_{\lambda}$ remains below one for realizations that we considered. In Figures $4.12(\mathrm{~b})$ and $(\mathrm{d})$, we see that $\log (\mathrm{SE}(\hat{\gamma}) / \hat{\gamma})$ varies between 1-5 for realizations of $\mathbf{D}_{i j}(\sigma)$ (in (b)) and between $-2-8$ for realizations of $\mathbf{D}_{i j}^{\text {rand }}(\sigma)$ (in (d)). 


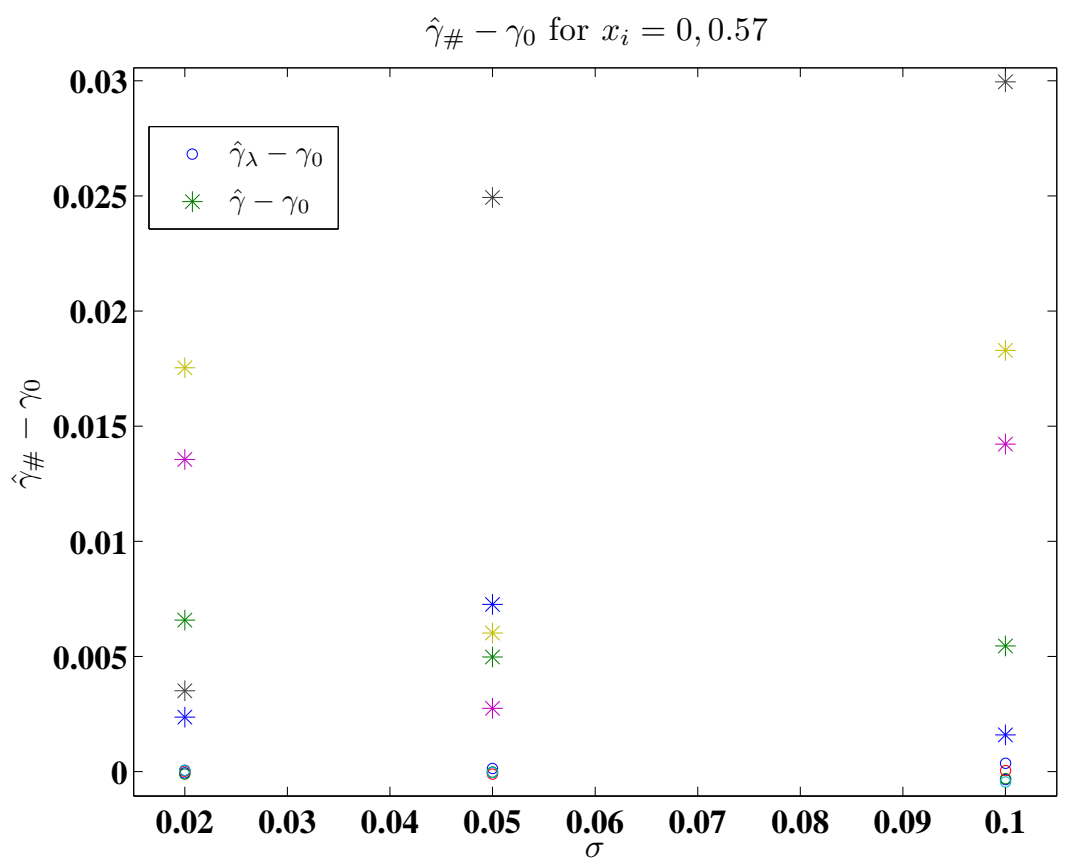

(a)

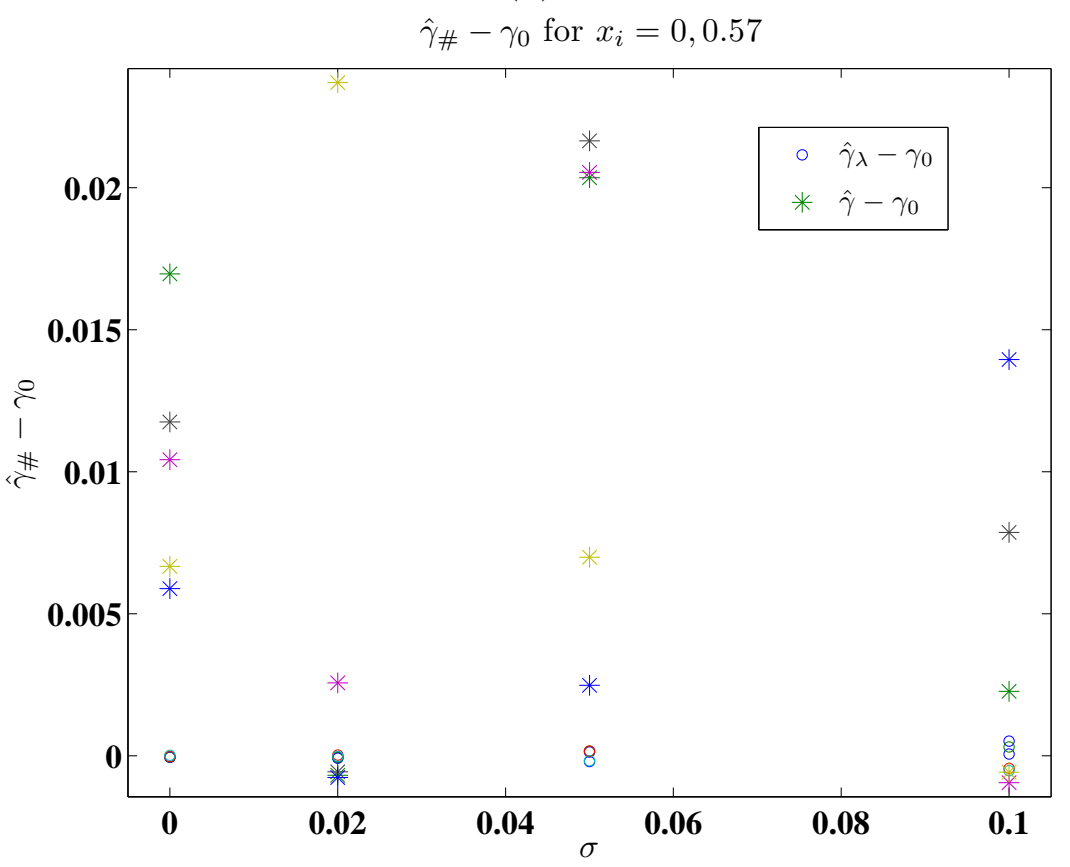

(b)

Figure 4.11: The results of realizations of simulated data with temporal nodes $t_{j}=0.6+$ $\frac{1}{120}, 0.6+\frac{2}{120}, \ldots, \frac{7}{120}, 20,40, \ldots, 140$ and spatial nodes $x_{i}=0,0.57$. The points denoted with ${ }^{\circ}$ are the difference $\hat{\gamma}_{\lambda}-\gamma_{0}$, and the points denoted ${ }^{*}$ are the difference $\hat{\gamma}-\gamma_{0}$ (a) The result of five realizations of $\mathbf{D}_{i j}(\sigma)$ for values of $\sigma=0.02,0.05,0.10$ (b) The result of five realizations of $\mathbf{D}_{i j}^{\text {rand }}(\sigma)$ for values of $\sigma=0,0.02,0.05,0.10$. 


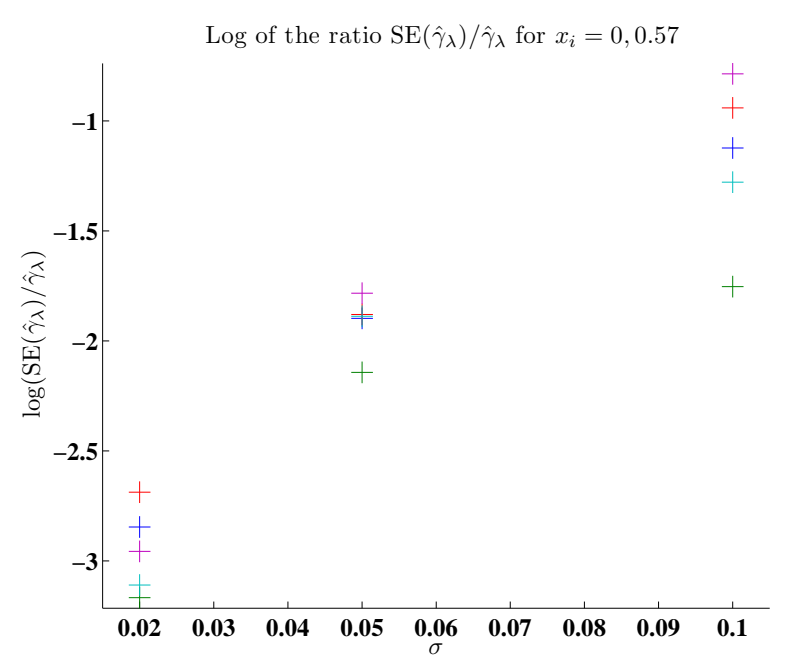

(a)

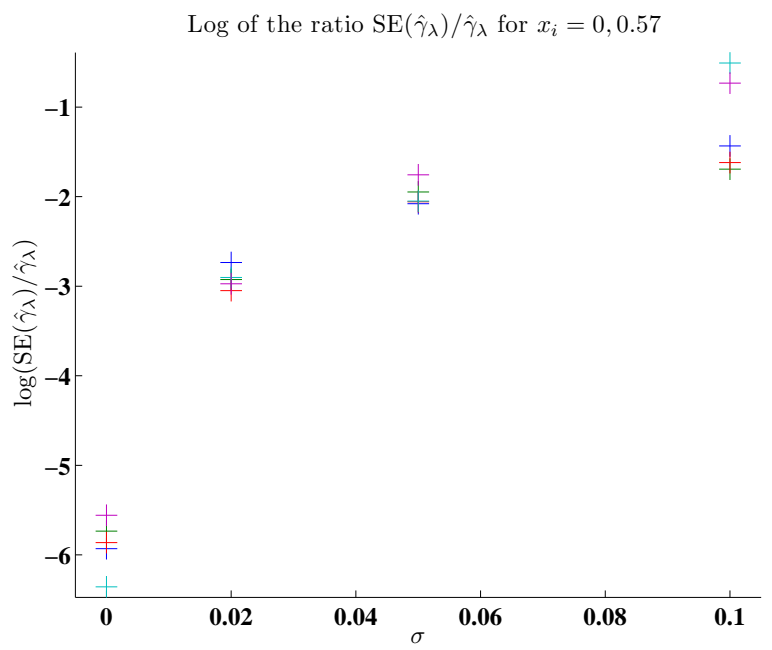

(c)

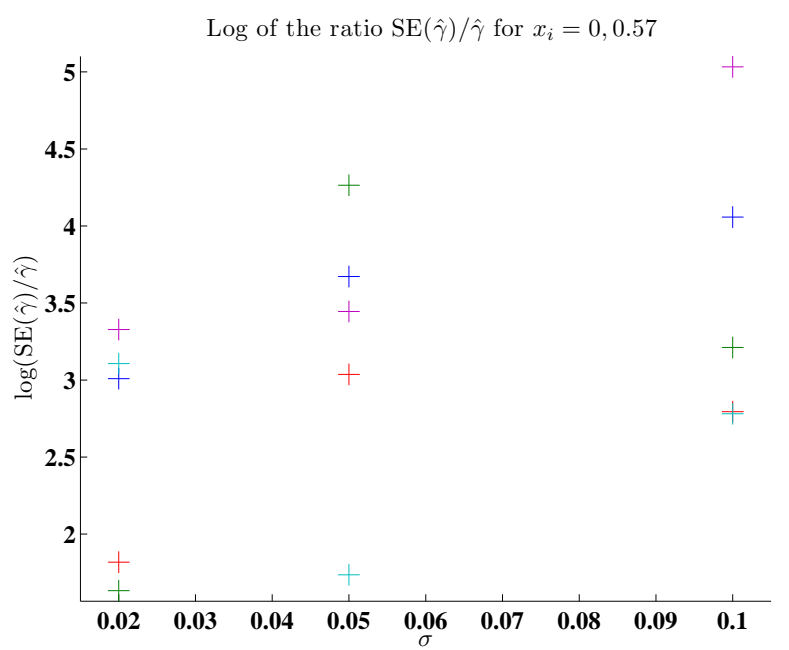

(b)

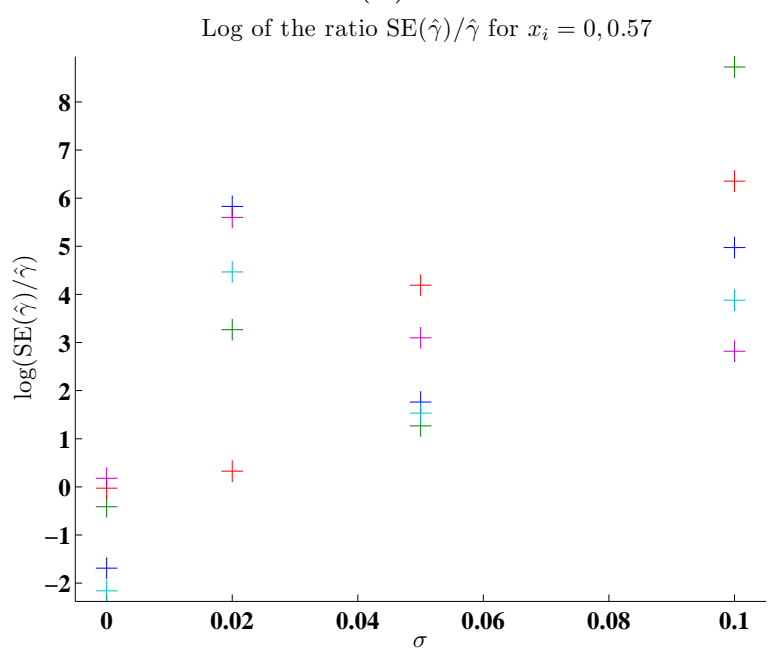

(d)

Figure 4.12: The results of realizations of simulated data with temporal nodes $t_{j}=0.6+$ $\frac{1}{120}, 0.6+\frac{2}{120}, \ldots, \frac{7}{120}, 20,40, \ldots, 140$ and spatial nodes $x_{i}=0,0.57$. (a) The ratio $\operatorname{SE}\left(\hat{\gamma}_{\lambda}\right) / \hat{\gamma}_{\lambda}$ for five realizations of $\mathbf{D}_{i j}(\sigma)$ for values of $\sigma=0.02,0.05,0.10$ (b) The log of the ratio $\log \operatorname{SE}(\hat{\gamma}) / \hat{\gamma}$ for five realizations of $\mathbf{D}_{i j}(\sigma)$ for values of $\sigma=0.02,0.05,0.10$. (c) The $\log$ of the ratio $\log \left(\operatorname{SE}\left(\hat{\gamma}_{\lambda}\right) / \hat{\gamma}_{\lambda}\right)$ for five realizations of $\mathbf{D}_{i j}^{\text {rand }}(\sigma)$ for values of $\sigma=0,0.02,0.05,0.10$. (d) The $\log$ of the ratio $\log \mathrm{SE}(\hat{\gamma}) / \hat{\gamma}$ for five realizations of $\mathbf{D}_{i j}^{\text {rand }}(\sigma)$ for values of $\sigma=$ $0,0.02,0.05,0.10$. 


\section{Conclusions}

We investigated in [2] the behavior of solutions of (2.2) (a partial differential equation on a homogeneous domain which is derived from homogenization theory) as approximations of solutions of (2.1) (a partial differential equation on a randomly perforated domain) in the forward problem. Here, we considered the behavior of solutions of (2.2) as approximations of solutions of (2.1) in associated inverse problems. Because it is often difficult to verify a priori the nature of random error (whether or not the error satisfies the OLS assumptions in (3.3) or the the GLS assumptions in (4.2)), we compared the efficacy of using solutions of (2.2) as a model solution for simulated data generated using solutions of (2.2) to the efficacy of using (2.2) as a model solution for simulated data generated using solutions of (2.1). The results were especially encouraging for the important parameter $\alpha$ (thermal diffusivity) which will be critical in our development of NDE methodology. The accuracy and uncertainty associated with the estimate of the parameter $\alpha$ was similar for data generated using (2.2) and (2.1) for data with relative and absolute added noise, for both sets of spatial nodes $x_{i}=0,0.57$ and $x_{i}=0,0.57,1.14$, and for parameter sets $\theta=(\gamma, \alpha, \lambda), \theta^{\gamma}=\left(\alpha_{\gamma}, \lambda_{\gamma}\right)$ and $\theta^{\lambda}=\left(\gamma_{\lambda}, \alpha_{\lambda}\right)$. Though estimating $\gamma$ presents difficulties in the inverse problem (adds uncertainty and inaccuracy to the estimate of $\lambda$ ), this affect was similar for data generated using solutions of (2.2) and data generated using solutions of (2.1). There was only one example in which there was a significant difference between using data generated using (2.2) and data generated using (2.1). For data with added absolute random error simulated at spatial nodes $x_{i}=0,0.57,1.14$, the uncertainty associated with the parameter estimate $\hat{\gamma}_{\lambda}$ was significantly larger for data generated using (2.1) than for data generated using (2.2). We believe the inverse problem findings in this report offer significant support that such methodologies as considered here will be most useful in development of NDE techniques for porous media structures.

\section{Acknowledgments}

This research was supported in part by the National Science Foundation under Research Training Grant (RTG) DMS-0636590 and in part by the Air Force Office of Scientific Research under grant number FA9550-09-1-0226.

\section{References}

[1] H. T. Banks, Brittany Boudreaux, Amanda Keck Criner, Krista Foster, Cerena Uttal, Thomas Vogel and William P. Winfree, Thermal based damage detection in porous materials, Inverse Problems in Science and Engineering, 18 (2009), 835-851.

[2] H T Banks, D. Cioranescu, A. K. Criner and W P Winfree, Modeling the flash-heat experiment on porous domains, Tech. Rep. CRSC-TR10-06, Center for Research in Scien- 
tific Computation, North Carolina State University, Raleigh, NC, May, 2010; Quarterly of Applied Mathematics, to appear.

[3] H. T. Banks, Sava Dediu, and S. L. Ernstberger, Sensitivity functions and their uses in inverse problems, Journal of Inverse and Ill-Posed Problems, 15 (2007), 683-708.

[4] H. T. Banks and B. G. Fitzpatrick, Statistical methods for model comparison in parameter estimation problems for distributed systems, Journal of Mathematical Biology, 28 (1990), 501-527.

[5] P. Donato and A. Nabil, Homogenization and correctors for the heat equation in perforated domains, Ricerche di Matematica, vol L, 1 (2001), 115-144.

[6] H. Thomas Banks, Marie Davidian, John R. Samuels, and Karyn L. Sutton, An inverse problem statistical methodology summary, Chap 11 in Mathematical and Statistical Estimation Approaches in Epidemiology, (Gerardo Chowell, et al., eds), Springer, Netherlands, 2009, pp. 249-302.

[7] H.T Banks, K. Holm, and F. Kappel, Comparison of optimal design methods in inverse problems. Tech. Rep. CRSC-TR10-11, Center for Research in Scientific Computation, North Carolina State University, Raleigh, NC, September, 2010; Inverse Problems, submitted.

[8] D. Cioranescu, A. Damlamian, and G. Griso, The periodic unfolding method in homogenization, SIAM Journal of Mathematical Analysis, 40 (2008), 1585-1620.

[9] D. Cioranescu, P. Donato, and R. Zaki, Asymptotic behavior of elliptic problems in perforated domains with nonlinear boundary conditions, Asymptotic Analysis, 53 (2007), 209-235.

[10] D. Cioranescu and J Saint Jean Paulin, Homogenization of Reticulated Structures, Vol 136, Applied Mathematical Sciences, Springer-Verlag, New York, 1999.

[11] Doina Cioranescu and Patrizia Donato, An Introduction to Homogenization, Oxford University Press, 1999.

[12] Doina Cioranescu, Patrizia Donato, and Rachad Zaki, The periodic unfolding method in perforated domains, Portugaliae Mathematica, 63 (2006), 467-496.

[13] M. Davidian and D.M. Giltinan, Nonlinear Models for Repeated Measurement Data, Chapman \& Hall, New York, 1995.

[14] The Mathworks, Inc., Partial Differential Equation Toolbox 1: User's Guide, 2008.

[15] Peter J. Shull, ed., Nondestructive Evaluation: Theory, Techniques, and Applications, Marcel Dekker, Inc., 2001. 
[16] W. J. Parker, R. J. Jenkins, C. P. Butler, and G. L. Abbott, Flash method of determining thermal diffusivity, heat capacity, and thermal conductivity, J. Appl. Phys., 32 (1961), 1679-1684.

[17] Pavel S̆olín, Partial Differential Equations and the Finite Element Method, John Wiley \& Sons, Inc, Hoboken, NJ, 2005.

[18] Wenping Wang, Jiaye Wang, and Myung-Soo Kim, An algebraic condition for the separation of two ellipsoids, Comput. Aided Geom. D., 18(2001), 531-539.

\section{A Sensitivity}

We use the finite element method to numerically solve (2.1) and (2.2). Here, we will discuss the numerical solution of (2.2) and the sensitivity functions in (3.7); see [1] for discussion of the numerical solution of (2.1). The finite element method approximates the infinite dimensional solution of a partial differential equation with a finite dimensional approximation. The domain $(\hat{\Omega})$ is discretized using the Delaunay triangulation. The finite dimensional solution is taken from the space of piecewise two dimensional affine functions, where the solution is affine on each mesh element (see [2], [14] and [17] for details). Specifically, in [2], we discussed the numerical approximation of $U$, the solution of $(2.2)$, given by $u^{N}(t, \vec{x})=\sum_{j=1}^{N} T_{j}(t) \phi_{j}(\vec{x})$ where $\phi_{j}(\vec{x})$ are piecewise affine basis element and $T_{j}(t)$ are their time dependent coefficients. The coefficients $T_{j}(t)$ are found by solving the ordinary differential equation for $\vec{T}(t)$ with entries $T_{j}(t)$

$$
p_{V} M \frac{d}{d t} \vec{T}(t)+\left(\alpha K+\lambda D+p_{V} \gamma M\right) \vec{T}(t)=S_{f} \mathcal{I}_{\left[t_{0}, t_{s}\right]}(t) \vec{f},
$$

where $M$ is an $N \times N$ positive definite matrix with elements $m_{i j}=\left\langle\phi_{i}, \phi_{j}\right\rangle, K$ is an $N \times N$ positive definite matrix with elements $k_{i j}=\left\langle\nabla \phi_{i}, A^{0} \nabla \phi_{j}\right\rangle, D$ is an $N \times N$ matrix with components $d_{i j}=\int_{\partial \widehat{\Omega}} \phi_{i} \phi_{j} d s, \vec{f}$ is an $N$-vector with components $f_{i}=\int_{\omega_{4}} \phi_{i}(x, 0) d x$ and $\vec{T}$ is an $N$ column vector. To approximate $U_{i j}\left(\theta^{\#}\right)$ in (3.1), we explicitly integrate the approximation $u^{N}\left(t_{j}, \vec{x}\right)$ which is piecewise affine on the source boundary $\omega_{4}$ so we use

$$
U_{i j}\left(\theta^{\#}\right) \approx \frac{1}{l} \int_{x_{i}}^{x_{i}+\ell} u^{N}\left(t_{j}, s, 0 ; \theta^{\#}\right) d s .
$$

Recall that in order to calculate the covariance matrices, we calculate the sensitivity matrix

$$
\chi_{i+m(j-1), k}(\theta)=\left.\frac{\partial}{\partial \zeta_{k}} U_{i j}(\zeta)\right|_{\zeta=\theta} .
$$

Throughout both the generalized least squares and ordinary least squares parameter estimation procedures, it is tacitly assumed that we use numerical estimations with reasonable convergence and that the admissible set of parameter is compact and finite dimensional (see 
[4] and [6]). Given these assumptions, in both (3.9) and (4.7), we may estimate $\chi$ with the derivative of the numerical solution itself. Explicitly, we use

$$
\chi_{i+m(j-1), k}(\theta) \approx \chi_{i+m(j-1), k}^{N}(\theta)=\left.\frac{\partial}{\partial \zeta_{k}}\left(\frac{1}{l} \int_{x_{i}}^{x_{i}+\ell} u^{N}\left(t_{j}, s, 0 ; \zeta\right) d s\right)\right|_{\zeta=\theta}
$$

Noting that the spatial nodes $x_{i}$ and the interval width $\ell$ are parameter independent, we may move the derivative under the integral and replace $u^{N}$ with its definition

$$
\left.\frac{\partial}{\partial \zeta_{k}}\left(\frac{1}{l} \int_{x_{i}}^{x_{i}+\ell} u^{N}\left(t_{j}, s, 0 ; \zeta\right) d s\right)\right|_{\zeta=\theta}=\left.\frac{1}{l} \int_{x_{i}}^{x_{i}+\ell} \frac{\partial}{\partial \zeta_{k}} \sum_{j=1}^{N} T_{j}(t ; \zeta) \phi_{j}(s, 0)\right|_{\zeta=\theta} d s
$$

Now, recalling that the basis elements $\phi_{j}$ are independent of $\theta$ and only $T_{j}(t ; \theta)$ are dependent on $\theta=(\gamma, \alpha, \lambda)$ in (A.1), we have

$$
\left.\frac{1}{l} \int_{x_{i}}^{x_{i}+\ell} \frac{\partial}{\partial \zeta_{k}} \sum_{j=1}^{N} T_{j}(t ; \zeta) \phi_{j}(s, 0)\right|_{\zeta=\theta} d s=\left.\frac{1}{l} \int_{x_{i}}^{x_{i}+\ell} \sum_{j=1}^{N} \phi_{j}(s, 0)\left(\frac{\partial}{\partial \zeta_{k}} T_{j}(t ; \zeta)\right)\right|_{\zeta=\theta} d s
$$

We need to solve for $\frac{\partial}{\partial \gamma} T_{j}(t ; \zeta), \frac{\partial}{\partial \alpha} T_{j}(t ; \zeta)$, and $\frac{\partial}{\partial \lambda} T_{j}(t ; \zeta)$. First, in order to calculate $\frac{\partial}{\partial \gamma} T_{j}(t ; \theta)$, we differentiate (A.1) with respect to $\gamma$. Let $\vec{T}_{\gamma}(t)$ denote $\frac{\partial}{\partial \gamma} \vec{T}(t)$, and recall that $M, K, D, \vec{f}$ are independent of $\gamma$. By the chain rule, $\frac{\partial}{\partial \gamma}\left(p_{V} \gamma M \vec{T}(t)\right)=p_{V} \gamma M \vec{T}_{\gamma}(t)+$ $p_{V} M \vec{T}(t)$, so differentiating (A.1) we obtain

$$
p_{V} M \frac{d}{d t} \vec{T}_{\gamma}(t)+\left(\alpha K+\lambda D+p_{V} \gamma M\right) \vec{T}_{\gamma}(t)+p_{V} M \vec{T}(t)=\overrightarrow{0}_{N \times 1}
$$

In (A.3) above, $M, K$ and $D$ are as defined in (A.1), and $\overrightarrow{0}_{N \times 1}$ is the $N \times 1$ vector of zeros. The ordinary differential equation (A.3) has a term that involves $\vec{T}(t)$, so (A.3) and (A.1) must be solved simultaneously. Similarly, we take the derivative of (A.1) with respect to $\alpha$ to obtain

$$
p_{V} M \frac{d}{d t} \vec{T}_{\alpha}(t)+\left(\alpha K+\lambda D+p_{V} \gamma M\right) \vec{T}_{\alpha}(t)+K \vec{T}(t)=\overrightarrow{0}_{N \times 1}
$$

which must also be solved with (A.1). Finally, we take the derivative of (A.1) with respect to $\lambda$ to find the system of ordinary differential equations for $\vec{T}_{\lambda}$ given by

$$
p_{V} M \frac{d}{d t} \vec{T}_{\lambda}(t)+\left(\alpha K+\lambda D+p_{V} \gamma M\right) \vec{T}_{\lambda}(t)+D \vec{T}(t)=\overrightarrow{0}_{N \times 1}
$$

which also must be solved simultaneously with (A.1). 